\title{
Investigating the effect of brittle crack propagation on the strength of ship structures by using peridynamics
}

\section{Cong Tien Nguyen and Selda Oterkus*}

Department of Naval Architecture, Ocean and Marine Engineering, University of Strathclyde, Glasgow, G11XQ, UK

\begin{abstract}
:
Ship structures can experience damages although they are initially designed with high safety factors. These damages can be caused by many reasons such as collisions, groundings, explosions, corrosion, fatigue, overloading or extreme conditions. Brittle damages can occur on the ship structures in some special conditions such as low-temperature, high-loading rate, multi-axial stress constraint, or low weldability of steel. In this study, progressive brittle damages on a ship structure subjected to different loading conditions are predicted. A novel peridynamic method based on a recently developed peridynamic shell model is presented to evaluate the structural strength during the damage evolution process for the first time in the literature. First, longitudinal strength (bending moment) of a ship structure is numerically calculated during damage propagations. The maximum longitudinal strength of the intact ship is verified with the experimental result. Next, the simulations are further extended to investigate the ship structure with initial damage, i.e. rectangular cut-out at the bottom. The longitudinal strength for an intact ship and a ship with different sizes of cut-outs are also compared. Finally, torsional strength during the damage propagation process for a ship structure with a cut-out is also presented.
\end{abstract}

Keywords: damage, ship structure, shells, longitudinal and torsional strengths, peridynamics

*Corresponding Author: Selda Oterkus, Department of Naval Architecture, Ocean and Marine Engineering, University of Strathclyde. Email: selda.oterkus@strath.ac.uk. 


\section{Introduction}

The safety of the ship and other marine structures is very important because their damages can cause many crucial issues. Therefore, these structures are often designed with high safety factors to minimize the potential of damages. However, according to the report by Allianz Global Corporate \& Specialty SE (2019), there are still 1036 total losses for vessels over 100GT (GT: Gross Tonnage) over the past 10 years.

The losses also happened to various types of vessels as shown in Fig. 1 (Allianz Global Corporate \& Specialty SE, 2019). The cargo ship has the highest number of total losses with 429 cases, followed by fishery and bulk vessels with 149 and 93 cases, respectively. The losses are also very common with passenger, chemical and container vessels. The reasons for ship losses are also very diverse as shown in Fig. 2 (Allianz Global Corporate \& Specialty SE, 2019), in which sinking, grounding, and explosion are three main reasons. Hull damage, collision and machine failure are also very common causes.

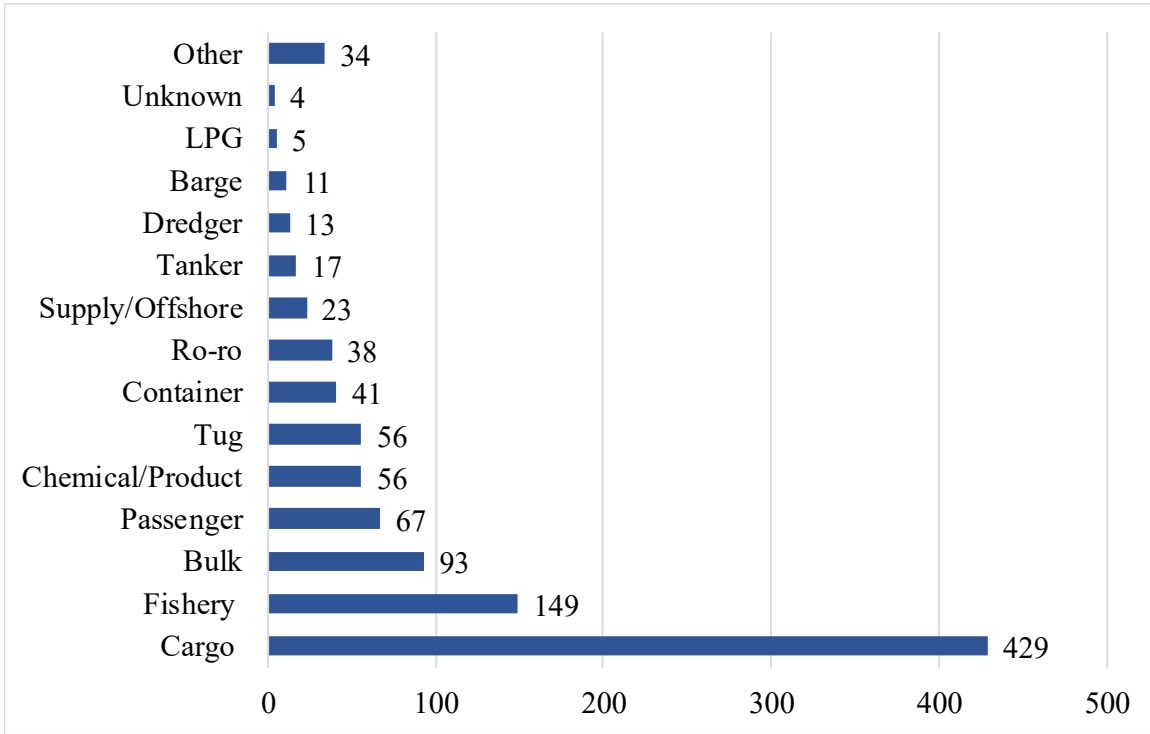

Fig. 1. Total losses by type of vessel over 100 Gross Tonnage from 2009 to 2018 (Allianz Global Corporate \& Specialty SE, 2019)

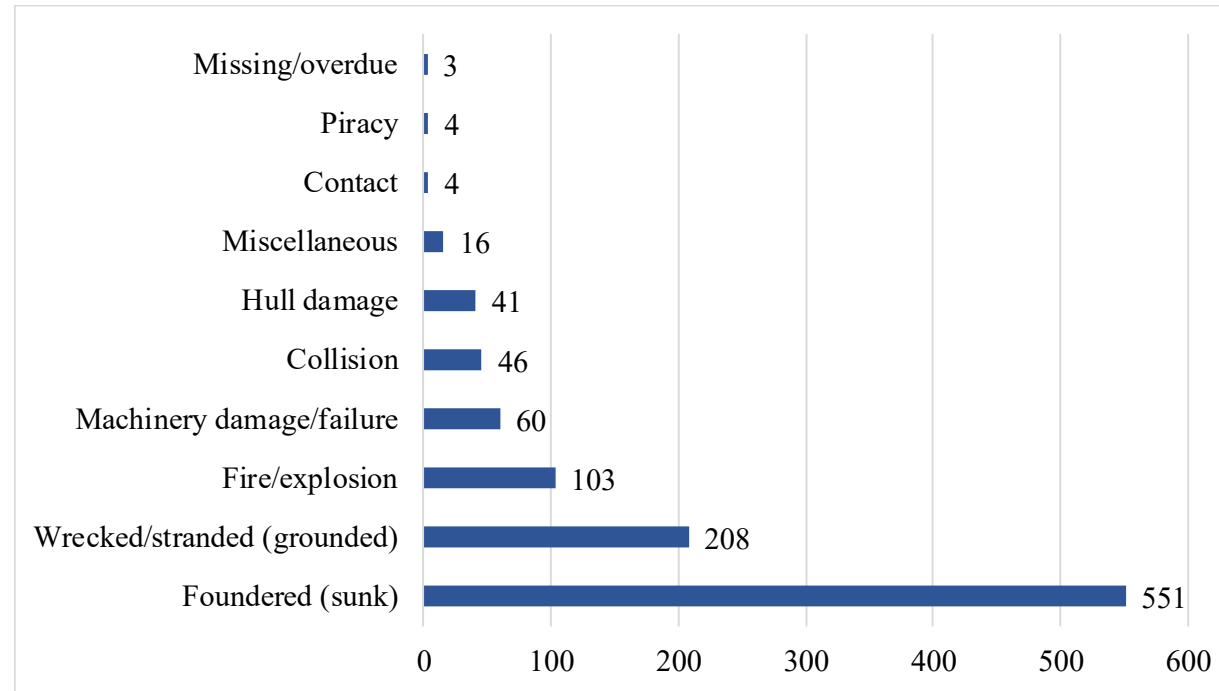

Fig. 2. Causes of losses from 2009 to 2018 for vessels over 100GT (Allianz Global Corporate \& Specialty SE, 2019) 
To date, how to minimize the ship losses is still a very challenging question that requires much effort to investigate. From the economic point of view, building a supper strong ship is not an optimal solution especially for merchant ships because it will increase the material cost as well as increase the weight of the ship. Therefore, understanding key factors that can help to minimize the structural damages is very crucial. One of the key tasks to do that is the ability to predict possible progressive damages and understanding the behaviors of the ship structures during the damage progress.

Ship structures can experience either brittle or ductile fractures. Ductile fractures are common damages on ship structures. Beyond the elastic limit of the steel, the ship structures can experience plastic response before being collapsed. These behaviors can be captured by using nonlinear finite element analysis and Elasto-Plastic Fracture Mechanics (AbuBakar and Dow, 2013, Simonsen and Törnquist, 2004, Benson et al., 2013, Paik et al., 2012, Kyokai, 2014).

Brittle fractures can also occur on a ship structure due to high cycle fatigue loading or when the ship is subjected to the conditions of low-temperature, high-loading rate, multi-axial stress constraint, or low weldability of steel (Sutar et al., 2014, Benac et al., 2016, Kobayashi and Onoue, 1943, Kim et al., 2016, Drouin, 2006).

Under the low-temperature conditions, steel can experience the ductile-to-brittle transition characteristics. Below the critical temperature, the material suddenly loses ductility and becomes brittle. Recently, Kim et al., (2016) also observed brittle fractures on a stiffened structure made of DH36 steel subjected to impact loading in $-60^{\circ} \mathrm{C}$ condition. This cold condition can be observed in Arctic environments during the winter in which the temperature can drop below $-50^{\circ} \mathrm{C}$ or even $-68^{\circ} \mathrm{C}$.

Poor fabrication practices, low material toughness, and poor design practices can also contribute to brittle failures. In South Africa, a pressurized ammonia bullet-type tank had not been stress relieved during the manufacturing, as well as after the welding process. As a result, the tank experienced a brittle fracture "in an explosive manner" when it was operating near ambient conditions (Benac et al., 2015, Benac et al., 2016). Later, the material experiments for this structure also showed poor toughness properties with the transition temperature above ambient conditions.

In 2002, the ship named Lake Carling experienced large brittle fractures on side shells when exposed to near $0^{0} \mathrm{C}$ temperatures. Later, the experiments indicated that steel from this vessel had very poor fracture arrest toughness (low Charpy Vee Notch energies) at temperatures near $0^{\circ} \mathrm{C}$. This caused a pre-existing crack at one frame of the ship to grow to failure at a load much lower than the ultimate tensile strength of the material (Drouin, 2006).

In this study, progressive brittle damage on a ship structure is predicted by a new nonlocal theory called "Peridynamics". Peridynamics (PD) represents the material behavior by using integro-differential equations that are valid in both continuous and discontinuous models (Silling, 2000, Silling and Askari, 2005). Therefore, progressive damages can be predicted by using a PD analysis without any special treatment. The first PD theory for three-dimensional (3D) solid objects was introduced by Silling (2000) and Silling and Askari (2005). The extensive literature surveys on peridynamics are given by Silling and Lehoucq (2010), Madenci and Oterkus (2014) and Javili et al. (2018).

Peridynamics can be used to analyze both elastic and inelastic material responses (Foster et al., 2010, Mitchell, 2011, Madenci and Oterkus, 2016a, Madenci and Oterkus, 2017, Huang et al., 2019). Moreover, it can also be either used to analyze composite and polycrystalline materials 
(Oterkus, 2010, Oterkus et al., 2012, Hu et al., 2012, Gao and Oterkus, 2019a, De Meo et al., 2016) or be applied for multiphysics (Alpay and Madenci, 2013, Oterkus et al., 2013, Oterkus, 2015, Madenci and Oterkus, 2016b, Gao and Oterkus, 2019b, 2019c) and multiscale modeling (Askari et al., 2008, Bobaru and Ha, 2011). Recently, Nguyen and Oterkus (2019a) developed an ordinary state-based PD model for geometrically nonlinear analysis by introducing a logarithmic bond stretch definition for large deformation problems.

Peridynamics can also be combined with finite element analysis (FEA) (Kilic and Madenci, 2010a, Oterkus et al., 2012, Liu and Hong, 2012, Bie et al., 2018) as well as implemented in FEA framework (Macek and Silling, 2007, Han et al., 2016, Diyaroglu et al., 2017, Yang et al., 2019).

Although the PD theory has many applications, developing a PD model for complex structures such as a ship and offshore structures is still a challenging work. When analyzing these complex structures, the existing 3D PD model becomes very computationally expensive. Therefore, simplified peridynamic structural models can be used to reduce the computational cost.

The first peridynamic model capturing tension and compression for 1D bars is introduced by Silling et al. (2003). Later, O'Grady and Foster (2014a) and Diyaroglu et al. (2015) introduced PD models for plane beam based on Euler-Bernoulli and Timoshenko beam theories, respectively. Moreover, Diyaroglu et al. (2019) developed an ordinary state-based PD model for the Euler-Bernoulli plane beam. Recently, Nguyen and Oterkus (2019c) developed a bondbased PD model for 3D beam structures with 6 degrees of freedom (DOF). The developed PD model can be used to predict progressive damages on offshore structures and any other type of beam structures.

Simplified models such as plate or shell models can be used for analyzing engineering structures. In peridynamics, the first simplified PD model for two-dimensional (2D) structures, which includes two in-plane degrees of freedom, was introduced by Silling and Bobaru (2005), and later by Madenci and Oterkus (2014), Breitenfeld et al. (2014), Sarego et al. (2016). In order to account for bending deformations, O'Grady and Foster (2014b) introduced a nonordinary state-based PD model based on Kirchhoff-Love plate theory. Later, Diyaroglu et al. (2015) also developed a bond based PD model for plate based on the Mindlin plate theory. The PD models developed by the authors can be applied for a single plate or flat shell. In practice, a shell structure can be constructed from many individual shells and plates. Therefore, a PD model that can capture the deformation of complex shell structures with 6 DOFs is necessary. For that purpose, Nguyen and Oterkus (2019b) recently developed an ordinary state-based PD model to predict thermomechanical behaviors of shell structures with 6 DOFs. The developed PD shell model can also be used to analyze behaviors of complex shell structures such as stiffened structures.

Therefore, this study focuses on predicting brittle damage progresses on ship structure by implementing the PD shell model developed by the authors. To demonstrate the capabilities of the PD model, first, damages on a plate subjected to anti-plane tearing, a plate with rectangular cut-out subjected to bending are predicted. Next, damage propagations for an intact ship structure subjected to bending loading conditions are investigated. For further investigation, the ship model is assumed to have initial damage, i.e. rectangular cut-out at the bottom, and the damage progress, as well as the strength of the ship, are investigated both for bending and torsional loading conditions. 
This paper is organized as follows. Section 2 presents the developed PD model for shell structures. Section 3 provides a damage criterion based on the critical energy rate for the PD shell model. The numerical algorithm to predict damages on complex shell structures is provided in Section 4. Section 5 presents the PD results, followed by the conclusions in Section 6.

\section{Peridynamic model for shell structures}

In this section, first, the PD equation of motion for shell structures in local coordinate is presented. Later, the PD equation of motion is transformed to its global form to analyze the complex shell structures.

\subsection{PD equations of motion in the local coordinate system}

Based on Mindlin plate theory, a flat plate element has 5 local degrees of freedom including three displacements $u, v, w$ and two rotations $\theta_{x}, \theta_{y}$ as shown in Fig. 3. The drilling rotation, $\theta_{z}$ for shell element can be introduced by adding a fictitious torsional stiffness matrix (Kanoknukulchai, 1979). Recently, (Nguyen and Oterkus, 2019b) developed a PD model that is capable of predicting damages in shell structures. Each material point in a shell has 6 global degrees of freedom including three displacements $u_{1}, u_{2}, u_{3}$ and three rotations $\theta_{1}, \theta_{2}, \theta_{3}$ as shown in Fig. 3. In Fig.3 $(x, y, z)$ represents the local coordinates and $\left(x_{1}, x_{2}, x_{3}\right)$ represents the global coordinates.

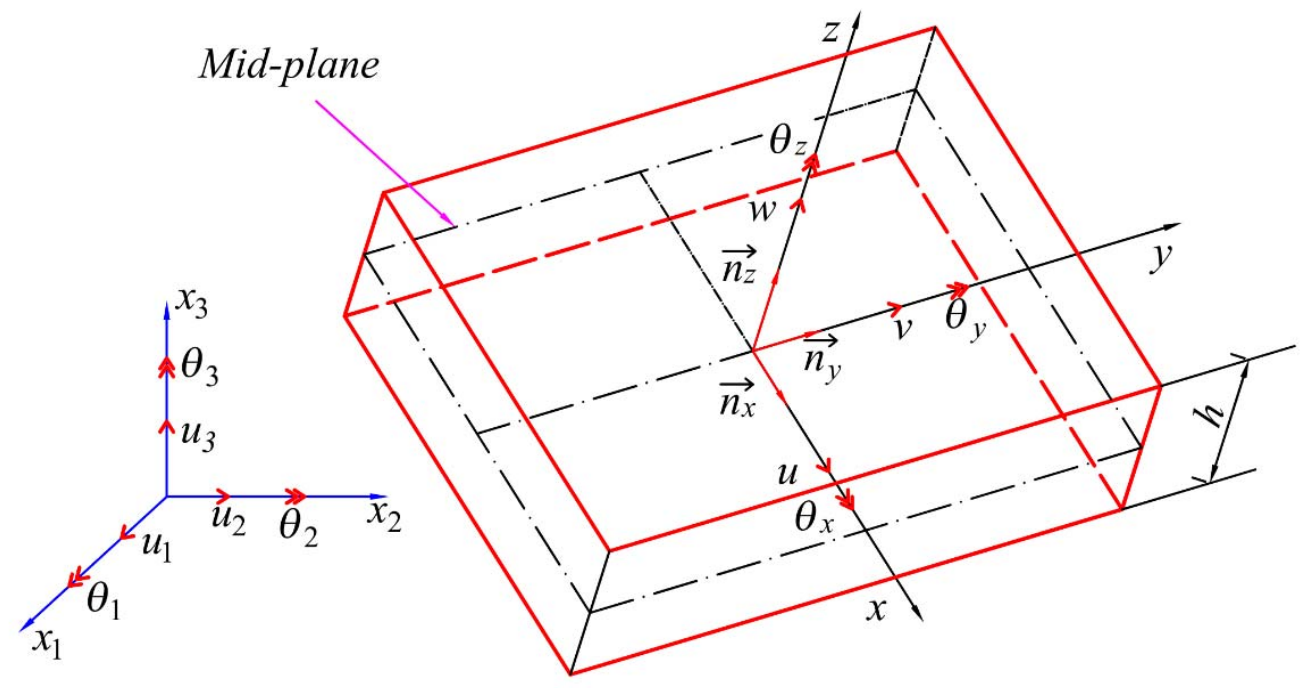

Fig. 3. Shell element and degrees of freedom for a material point

According to (Nguyen and Oterkus, 2019b), the PD equation of motion in the local coordinate system for a shell structure can be expressed as

$$
\overline{\mathbf{m}}_{(k)}^{L} \ddot{\mathbf{i}}_{(k)}^{L}=\sum_{j=1}^{N} \mu_{(k)(j)} \overline{\mathbf{f}}_{(k)(j)}^{L} V_{(j)}+\overline{\mathbf{b}}_{(k)}^{L}
$$

where $\mu_{(k)(j)}$ represents the interaction state, intact or broken, which can be defined as (Silling and Askari, 2005)

$$
\mu_{(k)(j)}\left(\mathbf{x}_{(j)}-\mathbf{x}_{(k)}, t\right)= \begin{cases}1 & \text { if interaction exists } \\ 0 & \text { if interaction is broken }\end{cases}
$$


In Eq. (1), the terms $\overline{\mathbf{m}}_{(k)}^{L}, \ddot{\mathbf{u}}_{(k)}^{L}$ and $\overline{\mathbf{b}}_{(k)}^{L}$ represent mass matrix, acceleration vector and vector of forces per unit area in local coordinates, respectively. These vectors and matrix can be defined as (Nguyen and Oterkus, 2019b)

$$
\overline{\mathbf{m}}_{(k)}^{L}=\left[\begin{array}{cccccc}
\rho h & 0 & 0 & 0 & 0 & 0 \\
0 & \rho h & 0 & 0 & 0 & 0 \\
0 & 0 & \rho h & 0 & 0 & 0 \\
0 & 0 & 0 & \frac{\rho h^{3}}{12} & 0 & 0 \\
0 & 0 & 0 & 0 & \frac{\rho h^{3}}{12} & 0 \\
0 & 0 & 0 & 0 & 0 & \rho h \frac{(\Delta x)^{4}}{6}
\end{array}\right] ; \ddot{\mathbf{i}}_{(k)}^{L}=\left[\begin{array}{c}
\ddot{u}_{(k)} \\
\ddot{v}_{(k)} \\
\ddot{w}_{(k)} \\
\ddot{\theta}_{x(k)} \\
\ddot{\theta}_{y(k)} \\
\ddot{\theta}_{z(k)}
\end{array}\right] ; \overline{\mathbf{b}}_{(k)}^{L}=\left[\begin{array}{c}
\bar{b}_{x(k)} \\
\bar{b}_{y(k)} \\
\bar{b}_{z(k)} \\
\bar{m}_{x(k)} \\
\bar{m}_{y(k)} \\
0
\end{array}\right]
$$

where $h$ represents the thickness of the shell, $\Delta x$ represents uniform mesh size in the PD discretized model, $\bar{b}_{x(k)}, \bar{b}_{y(k)}, \bar{b}_{z(k)}$ represent applied forces per unit area and $\bar{m}_{x(k)}, \bar{m}_{y(k)}$ represent applied bending moments per unit area (Nguyen and Oterkus, 2019b).

The term $\overline{\mathbf{f}}_{(k)(j)}^{L}$ in Eq. (1) represents the vector of PD forces which can be defined as (Nguyen and Oterkus, 2019b)

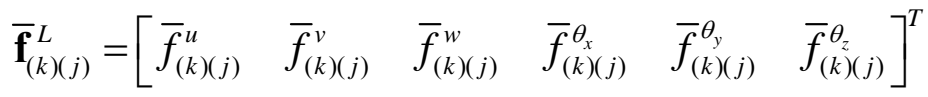

with

$$
\begin{aligned}
\bar{f}_{(k)(j)}^{u}= & {\left[2 a_{i p} d_{i p} \frac{1}{\xi}\left(\vartheta_{(k)}+\vartheta_{(j)}\right)+4 b_{i p} s_{i p(k)(j)}\right] \cos \varphi } \\
\bar{f}_{(k)(j)}^{v}= & {\left[2 a_{i p} d_{i p} \frac{1}{\xi}\left(\vartheta_{(k)}+\vartheta_{(j)}\right)+4 b_{i p} s_{i p(k)(j)}\right] \sin \varphi } \\
\bar{f}_{(k)(j)}^{w}= & C_{s}\left\{\frac{w_{(j)}-w_{(k)}}{\xi}-\frac{1}{2}\left[-\left(\theta_{y(k)}+\theta_{y(j)}\right) \cos \varphi+\left(\theta_{x(k)}+\theta_{x(j)}\right) \sin \varphi\right]\right\} \\
\bar{f}_{(k)(j)}^{\theta_{x}}= & {\left[\frac{2 a_{b} d_{b}}{\xi}\left(\vartheta_{b(k)}+\vartheta_{b(j)}\right)+4 b_{b} s_{b(k)(j)}\right] \sin \varphi } \\
& +\frac{1}{2} C_{s}\left\{\left(w_{(j)}-w_{(k)}\right) \sin \varphi-\frac{\xi}{2}\left[-\left(\theta_{y(k)}+\theta_{y(j)}\right) \sin \varphi \cos \varphi\right]\right. \\
\bar{f}_{(k)(j)}^{\theta_{y}}= & -\left[\frac{2 a_{b} d_{b}}{\xi}\left(\vartheta_{b(k)}+\vartheta_{b(j)}\right)+4 b_{b} s_{b(k)(j)}\right) \sin { }^{2} \varphi \\
& -\frac{1}{2} C_{s}\left\{\left(w_{(j)}-w_{(k)}\right) \cos \varphi-\frac{\xi}{2}\left[-\left(\theta_{y(k)}+\theta_{y(j)}\right) \cos { }^{2} \varphi\right.\right. \\
& \left.\left.\left(\theta_{x(k)}+\theta_{x(j)}\right) \sin \varphi \cos \varphi\right]\right\} \\
\bar{f}_{(k)(j)}^{\theta_{z}}= & C_{t}\left[\frac{\theta_{z(k)}+\theta_{z(j)}}{2}-\left(\frac{v_{(j)}-v_{(k)}}{\xi} \cos \varphi-\frac{\left.\left.u_{(j)}-u_{(k)} \sin \varphi\right)\right] \xi}{\xi}\right)\right.
\end{aligned}
$$


In Eqs. (4b) and (4c), $a_{i p}, b_{i p}, d_{i p}$ represent PD constants for in-plane deformations which can be defined as (Nguyen and Oterkus, 2019b)

$$
\begin{aligned}
& a_{i p}=\frac{E h(3 v-1)}{4\left(1-v^{2}\right)} \\
& b_{i p}=\frac{3}{\pi \delta^{3}} \frac{E}{1+v} \\
& d_{i p}=\frac{2}{\pi h \delta^{2}}
\end{aligned}
$$

The terms $\vartheta_{(k)}$ and $\vartheta_{(j)}$ represent dilatations of material points $k$ and $j$, respectively. These terms can be defined as (Nguyen and Oterkus, 2019b)

$$
\vartheta_{(k)}=d_{i p} \sum_{j=1}^{N} \mu_{(k)(j)} S_{i p(k)(j)} V_{(j)}
$$

where $s_{i p(k)(j)}$ represents the relative bond stretch for in-plane deformations which can be defined as (Nguyen and Oterkus, 2019b, Diyaroglu, 2016, Silling and Lehoucq, 2010)

$$
s_{i p(k)(j)}=\frac{\left(u_{(j)}-u_{(k)}\right) \cos \varphi+\left(v_{(j)}-v_{(k)}\right) \sin \varphi}{\xi}
$$

with

$$
\begin{aligned}
& \cos \varphi=\frac{x_{(j)}-x_{(k)}}{\xi} \\
& \sin \varphi=\frac{y_{(j)}-y_{(k)}}{\xi} \\
& \xi=\sqrt{\left(x_{(j)}-x_{(k)}\right)^{2}+\left(y_{(j)}-y_{(k)}\right)^{2}}
\end{aligned}
$$

where $\varphi$ represents the angle between the interaction between material points $k$ and $j$ with the local axis, $x_{1}$ in the undeformed configuration and $\xi$ represents the distance between material points $k$ and $j$.

In Eq. (4d), $C_{s}$ represents PD constant for shear deformations of shell structure which can be represented as (Nguyen and Oterkus, 2019b)

$$
C_{s}=\frac{3 k_{s}}{\pi \delta^{3}} \frac{E}{(1+v)}
$$

with

$$
k_{\mathrm{s}}=\frac{5}{6}
$$

In Eqs. (4e) and (4f), $a_{b}, b_{b}$ and $d_{b}$ represent PD constants for bending deformations which can be represented as (Nguyen and Oterkus, 2019b)

$$
\begin{aligned}
& a_{b}=\frac{E h^{3}}{48} \frac{3 v-1}{1-v^{2}} \\
& b_{b}=\frac{E h^{2}}{4(1+v) \pi \delta^{3}}
\end{aligned}
$$


$d_{b}=\frac{2}{\pi h \delta^{2}}$

The terms $\vartheta_{b(k)}$ and $s_{b(k)(j)}$ in Eq. (4e) and (4f) are defined as (Nguyen and Oterkus, 2019b)

$\vartheta_{b(k)}=d_{b} \sum_{j=1}^{N} \mu_{(k)(j)} s_{b(k)(j)} V_{(j)}$

$s_{b(k)(j)}=\frac{-\left(\theta_{y(j)}-\theta_{y(k)}\right) \cos \varphi+\left(\theta_{x(j)}-\theta_{x(k)}\right) \sin \varphi}{\xi}$

In Eq. (4g), $C_{t}$ represents PD constant for torsional deformations of shells which can be represented as (Nguyen and Oterkus, 2019b)

$C_{t}=k_{T} \frac{3}{2 \pi \delta^{3}} \frac{E}{1+v}$

with

$k_{T}=\frac{h^{2}}{6}$

Note that the PD force component, $\bar{f}_{(k)(j)}^{\theta_{2}}$ given in Eq. $(4 \mathrm{~g})$ is fictitious which is added to the vector of PD forces, $\overline{\mathbf{f}}_{(k)(j)}^{L}$ to generate a small stiffness for the drilling rotation (Nguyen and Oterkus, 2019b, Kanok-nukulchai, 1979).

As given in Eq. (1), the external loads are applied to the PD shell model by using forces and bending moments per unit area. Forces and bending moments can also be directly used in this PD model by multiplying both sides of Eq. (1) with the area of a material point, $A_{(k)}$ as

$\left(A_{(k)} \overline{\mathbf{m}}_{(k)}^{L}\right) \ddot{\mathbf{u}}_{(k)}^{L}=\sum_{j=1}^{N} \mu_{(k)(j)} \overline{\mathbf{f}}_{(k)(j)}^{L} A_{(k)} V_{(j)}+A_{(k)} \overline{\mathbf{b}}_{(k)}^{L}$

or

$\mathbf{M}_{(k)}^{L} \ddot{\mathbf{u}}_{(k)}^{L}=\sum_{j=1}^{N} \mu_{(k)(j)} \mathbf{F}_{(k)(j)}^{L} V_{(j)}+\mathbf{B}_{(k)}^{L}$

with

$\mathbf{M}_{(k)}^{L}=A_{(k)} \overline{\mathbf{m}}_{(k)}^{L}$

$\mathbf{F}_{(k)(j)}^{L}=\overline{\mathbf{f}}_{(k)(j)}^{L} A_{(k)}$

$\mathbf{B}_{(k)}^{L}=\overline{\mathbf{b}}_{(k)}^{L} A_{(k)}$

where the term $\mathbf{B}_{(k)}^{L}$ represents a vector of applied forces and bending moments.

2.2. PD equations of motion in the global coordinate system

In order to investigate the behaviors of complex shell structures, the PD equation of motion in local coordinates for shell given in Eq. (12b) can be transformed to the global coordinates system (Nguyen and Oterkus, 2019b, 2019c).

The orientation of a shell element for a material point can be defined by director vectors, $\vec{n}_{x(k)}$ $, \vec{n}_{y(k)}, \vec{n}_{z(k)}$ as shown in Fig.3. Since different material points may have different director vectors, the average director vectors for interaction between material points $k$ and $j$ can be estimated as (Nguyen and Oterkus, 2019b, 2019c) 


$$
\begin{aligned}
& \vec{n}_{x(k)(j)}=\frac{\left(\vec{n}_{x(k)}+\vec{n}_{x(j)}\right)}{\left|\left(\vec{n}_{x(k)}+\vec{n}_{x(j)}\right)\right|} \\
& \vec{n}_{y(k)(j)}=\frac{\left(\vec{n}_{y(k)}+\vec{n}_{y(j)}\right)}{\left|\left(\vec{n}_{y(k)}+\vec{n}_{y(j)}\right)\right|} \\
& \vec{n}_{z(k)(j)}=\frac{\left(\vec{n}_{z(k)}+\vec{n}_{z(j)}\right)}{\left|\left(\vec{n}_{z(k)}+\vec{n}_{z(j)}\right)\right|}
\end{aligned}
$$

where $\vec{n}_{x(j)}, \vec{n}_{y(j)}, \vec{n}_{z(j)}$ represent director vectors for material point $j$. Therefore, the relations between local and global coordinates can be represented as (Nguyen and Oterkus, 2019b, 2019c)

$\left[\begin{array}{lll}x & y & z\end{array}\right]^{T}=\mathbf{H}_{(k)(j)}\left[\begin{array}{lll}x_{1} & x_{2} & x_{3}\end{array}\right]^{T}$

with

$$
\mathbf{H}_{(k)(j)}=\left[\begin{array}{lll}
\vec{n}_{x(k)(j)} & \vec{n}_{y(k)(j)} & \vec{n}_{z(k)(j)}
\end{array}\right]^{T}
$$

where $\mathbf{H}_{(k)(j)}$ represents coordinate transformation matrix for each interaction. Therefore, the relations between local and global degrees of freedom can be represented as (Nguyen and Oterkus, 2019b, 2019c)

$$
\mathbf{u}_{(k)}^{L}=\mathbf{T}_{(k)(j)} \mathbf{u}_{(k)}^{G}
$$

with

$$
\mathbf{T}_{(k)(j)}=\left[\begin{array}{cc}
\mathbf{H}_{(k)(j)} & \mathbf{0} \\
\mathbf{0} & \mathbf{H}_{(k)(j)}
\end{array}\right]
$$

where $\mathbf{T}_{(k)(j)}$ represents the displacement transformation matrix. The parameter $\mathbf{u}_{(k)}^{G}$ represents the global degrees of freedom vector as

$$
\mathbf{u}_{(k)}^{G}=\left[\begin{array}{llllll}
u_{1} & u_{2} & u_{3} & \theta_{1} & \theta_{2} & \theta_{3}
\end{array}\right]^{T}
$$

The equation of motion given in Eq. (12b) can be transformed into the global coordinate system by multiplying both sides by $\mathbf{T}_{(k)(j)}^{T}$ as (Nguyen and Oterkus, 2019b)

$$
\left(\mathbf{T}_{(k)(j)}^{T} \mathbf{M}_{(k)}^{L} \mathbf{T}_{(k)(j)}\right) \ddot{\mathbf{u}}_{(k)}^{G}=\sum_{j=1}^{N} \mu_{(k)(j)}\left(\mathbf{T}_{(k)(j)}^{T} \mathbf{F}_{(k)(j)}^{L}\right) V_{(j)}+\mathbf{T}_{(k)(j)}^{T} \mathbf{B}_{(k)}^{L}
$$

or

$$
\mathbf{M}_{(k)}^{G} \ddot{\mathbf{i}}_{(k)}^{G}=\sum_{j=1}^{N} \mu_{(k)(j)} \mathbf{F}_{(k)(j)}^{G} V_{(j)}+\mathbf{B}_{(k)}^{G}
$$

with

$$
\begin{aligned}
& \mathbf{M}_{(k)}^{G}=\mathbf{T}_{(k)(j)}^{T} \mathbf{M}_{(k)}^{L} \mathbf{T}_{(k)(j)} \\
& \mathbf{F}_{(k)(j)}^{G}=\mathbf{T}_{(k)(j)}^{T} \mathbf{F}_{(k)(j)}^{L} \\
& \mathbf{B}_{(k)}^{G}=\mathbf{T}_{(k)(j)}^{T} \mathbf{B}_{(k)}^{L}
\end{aligned}
$$

where $\mathbf{M}_{(k)}^{G}, \mathbf{F}_{(k)(j)}^{G}$ and $\mathbf{B}_{(k)}^{G}$ represent mass matrix, the vector of PD force densities and vector of applied forces and bending moments in the global coordinates, respectively. 


\section{Damage prediction}

The damages on shell structures can be predicted by using the criterion based on the critical energy release rate (Madenci and Oterkus, 2016a, 2017, Nguyen and Oterkus, 2019b, 2019c). The state of each interaction can be determined based on the comparison between the energy release rate and its critical value. If the energy release rate of interaction exceeds its critical value, the interaction is considered as irreversibly broken which leads to crack growth. This criterion can be used to determine function, $\mu_{(k)(j)}$ as (Madenci and Oterkus, 2016a, 2017)

$\bar{g}_{(k)(j)}<g_{c} \rightarrow$ interaction exists: $\mu_{(k)(j)}=1$

$\bar{g}_{(k)(j)} \geq g_{c} \rightarrow$ interaction is broken: $\mu_{(k)(j)}=0$

where $g_{c}$ represents the average critical energy release rate for one interaction which can be computed as (Madenci and Oterkus, 2016a, 2017)

$g_{c}=G_{c} / N_{c}$

where $G_{c}$ represents the critical energy release rate of material and $N_{c}$ represents the total number of interactions passing through a unit crack area, $A_{0}$. With a horizon size of $\delta=3.015 \Delta x$, this number can be counted as $N_{c}=36$ (Nguyen and Oterkus, 2019a, 2019b). The unit crack area, $A_{0}$, for shell structure can be defined as

$$
A_{0}=h(\Delta x)
$$

The term $\bar{g}_{(k)(j)}$ in Eq. (18) represents the energy release rate for interaction between material points $k$ and $j$ which can be calculated as

$$
\bar{g}_{(k)(j)}=\frac{1}{2}\left(g_{(k)(j)}+g_{(j)(k)}\right)
$$

with

$$
\begin{aligned}
& g_{(k)(j)}=\frac{1}{A_{0}} \omega_{(k)(j)} V_{(k)} V_{(j)} \\
& g_{(j)(k)}=\frac{1}{A_{0}} \omega_{(j)(k)} V_{(j)} V_{(k)}
\end{aligned}
$$

where $\omega_{(k)(j)}$ and $\omega_{(j)(k)}$ represent micropotentials of the interaction between material points $k$ and $j$ which can be calculated as (Nguyen and Oterkus, 2019b)

$$
\omega_{(k)(j)}=\omega_{\text {inplane }(k)(j)}+\omega_{\text {shear }(k)(j)}+\omega_{\text {bending }(k)(j)}
$$

where $\omega_{\text {inplane }(k)(j)}, \omega_{\text {shear }(k)(j)}, \omega_{\text {bending }(k)(j)}$ represent micropotentials for in-plane, shear, and bending deformations, respectively.

In linear elasticity for small deformations, the micropotential for in-plane deformations, $\omega_{\text {inplane(k)(j) }}$, can be calculated as (Nguyen and Oterkus, 2019b)

$$
\omega_{\text {inplane }(k)(j)}=\frac{1}{2 h} t_{i p(k)(j)} \xi S_{i p(k)(j)}
$$

with

$$
t_{i p(k)(j)}=\left[\frac{2 a_{i p} d_{i p}}{\xi} \vartheta_{(k)}+2 b_{i p} s_{i p(k)(j)}\right]
$$

Similarly, the micropotential for bending deformations, $\omega_{\text {bending }(k)(j)}$, can be calculated as

$$
\omega_{\text {bending }(k)(j)}=\frac{1}{2 h} t_{b(k)(j)} \xi s_{b(k)(j)}
$$


with

$$
t_{b(k)(j)}=\left[\frac{2 a_{b} d_{b}}{\xi} \vartheta_{b(k)}+2 b_{b} s_{b(k)(j)}\right]+\frac{1}{4} C_{s}\left\{\left(w_{(j)}-w_{(k)}\right)-\frac{\xi}{2}\left[\begin{array}{l}
-\left(\theta_{y(k)}+\theta_{y(j)}\right) \cos \varphi \\
+\left(\theta_{x(k)}+\theta_{x(j)}\right) \sin \varphi
\end{array}\right]\right\}
$$

On the other hand, the micropotential for shear deformations, $\omega_{\text {shear }(k)(j)}$, can be calculated as

$\omega_{\text {shear }(k)(j)}=\frac{1}{2 h} t_{\text {shear }(k)(j)} \xi S_{\text {shear }(k)(j)}$

with

$t_{\text {shear }(k)(j)}=\frac{1}{2} C_{s}\left\{\frac{w_{(j)}-w_{(k)}}{\xi}-\frac{1}{2}\left[-\left(\theta_{y(k)}+\theta_{y(j)}\right) \cos \varphi+\left(\theta_{x(k)}+\theta_{x(j)}\right) \sin \varphi\right]\right\}$

and

$S_{\text {shear }(k)(j)}=\frac{w_{(j)}-w_{(k)}}{\xi}$

The damages on the structure can be represented by the local damage, $\phi$, which is the ratio of broken interactions to the total number of interactions associated with a material point within its horizon. This parameter is represented as (Silling and Askari, 2005)

$$
\phi\left(\mathbf{x}_{(k)}, t\right)=1-\frac{\sum_{j=1}^{N} \mu_{(k)(j)}\left(\mathbf{x}_{(j)}-\mathbf{x}_{(k)}, t\right) V_{(j)}}{\sum_{j=1}^{N} V_{(j)}}
$$

\section{Numerical procedure}

The PD equation of motion given in Eq. (17b) can be solved by using a meshless scheme. The domain is discretized by material points. Each material point has its volume, thickness, and its director vectors. The PD equation of motion can be solved by using explicit time integration. The numerical procedure is shown in Fig. 4. 


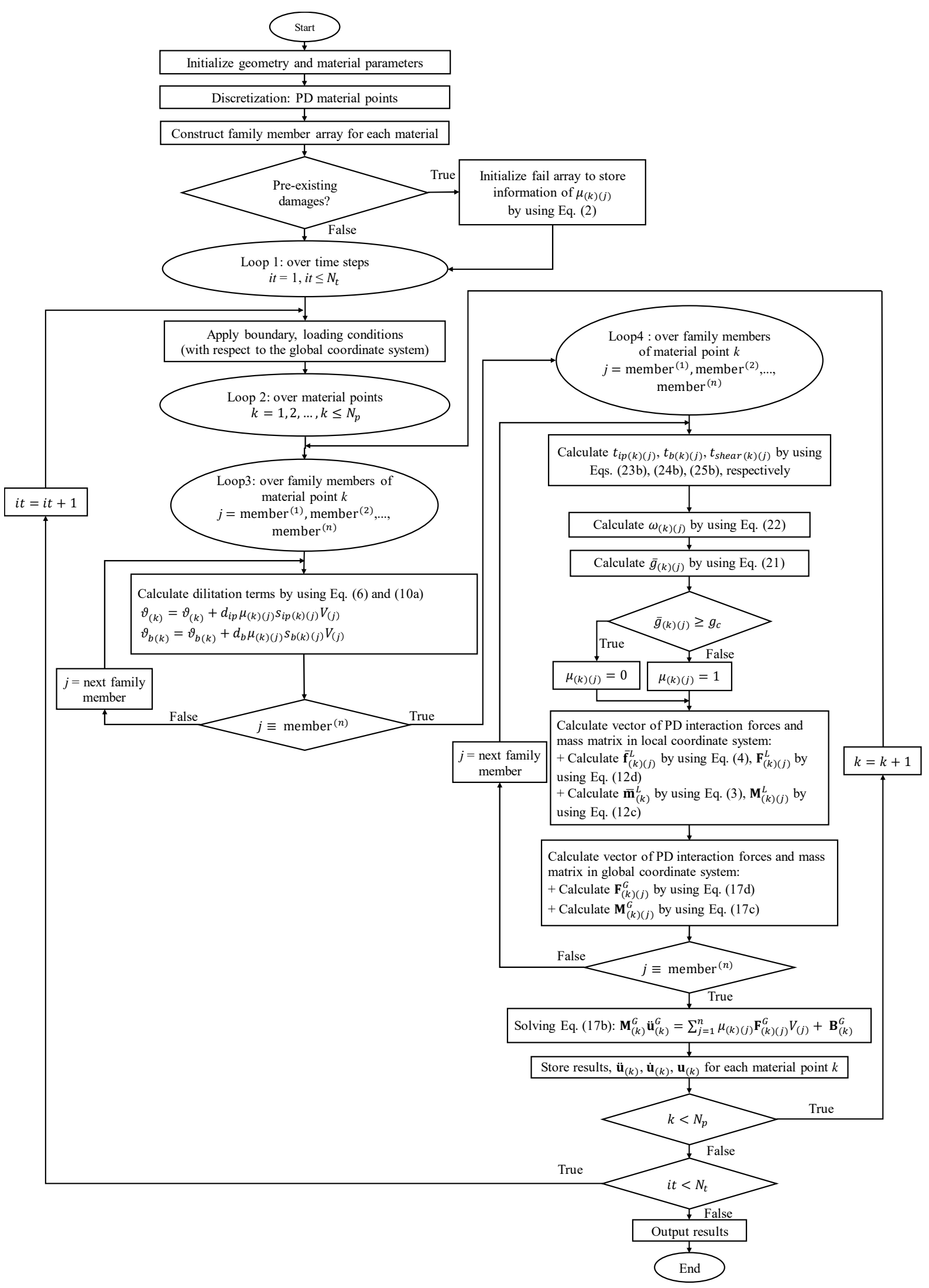

Fig. 4. Numerical procedure

\section{Numerical results}

In this section, first, the PD shell model is used to predict damages on a plate subjected to antiplane tearing. Then, damage propagation on a plate with rectangular cut-out subjected to 
bending is predicted. Finally, progressive damages on a ship structure subjected to bending and torsional loadings are predicted. Since the effects of near-surface boundaries are significant in the PD models, the surface corrections are adopted based on the method described by Madenci and Oterkus, 2014.

\subsection{Plate subjected to anti-plane tearing}

As shown in Fig.5, a plate with dimensions of $L=0.0762 \mathrm{~m}, B=0.0635 \mathrm{~m}$, and thickness of $h=0.0025 \mathrm{~m}$, subjected to anti-plane tearing is investigated. The plate has an initial crack located at $x_{2}=0$ with a crack length of $a=0.0334 \mathrm{~m}$ (Li et al., 2011). Both sides of the crack are constrained with rigid plates with a size of $l=21.5 \times 10^{-3} \mathrm{~m}$.

The plate is made of Aluminium alloy with Young's modulus $E=68.9 \times 10^{9} \mathrm{~N} / \mathrm{m}^{2}$, Poisson's ratio $v=0.33$, fracture toughness $K_{c}=48.7 \times 10^{6} \mathrm{Nm}^{-3 / 2}$ (MacMaster et al., 2000) and critical energy release rate $G_{c}=3.4422 \times 10^{4} \mathrm{~J} / \mathrm{m}^{2}$. The rigid plates are incrementally moved apart from each other to represent the anti-plane tearing condition (Li et al., 2011).

In the PD model, the plate is uniformly discretized with a mesh size of $\Delta x=6.35 \times 10^{-4} \mathrm{~m}$. As utilised by Diyaroglu et al. (2015) and Nguyen and Oterkus, (2019b), accurate results for the behaviors of plates and shells are obtained by using the PD model with the horizon size of $\delta=3.015 \Delta x$. Therefore, in this study, the horizon size of $\delta=3.015 \Delta x$ is used. In order to apply the loading, the displacement loading is increased at each load step by $\left|\Delta u_{3}\right|=10^{-8} \mathrm{~m}$. The displacement components of two material points located at $\left(x_{1}=L / 2, x_{2}=-B / 2\right)$ and $\left(x_{1}=L / 2, x_{2}=B / 2\right)$ are set equal to zero. The PD solution for the quasi-static loading condition is obtained by using the adaptive dynamic relaxation (ADR) method (Kilic and Madenci, 2010b; Underwood, 1986).

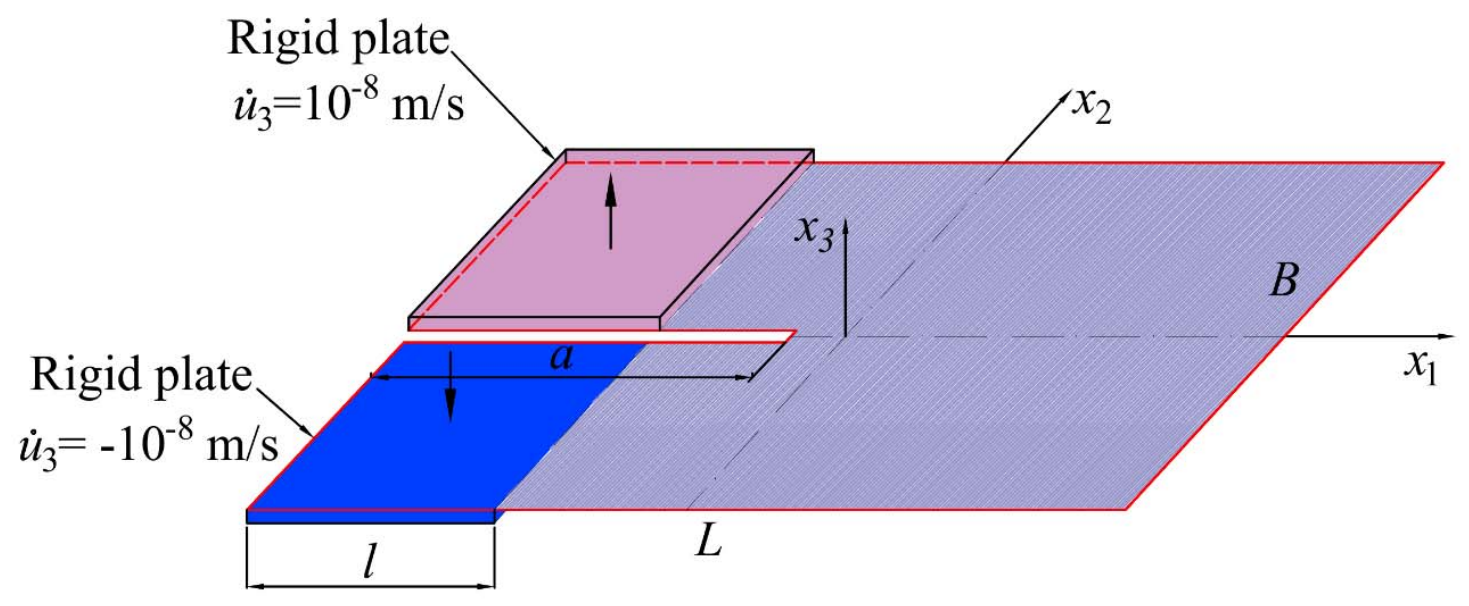

Fig. 5. The geometrical model for anti-plane tearing of a plate

Fig. 6 presents the crack propagation pattern for different load steps. In this figure, the color plots represent the local damage parameter shown in Eq. (26). It is observed that the damage starts to propagate at loading $u_{3}= \pm 3.9283 \times 10^{-4} \mathrm{~m}$ as shown in Fig. 6(a). As the applied displacements increase, the crack propagates toward the right edge of the plate as shown in Figs. 6(b-d). It is observed that the crack path captured by the PD shell model agrees with the experimental results obtained by Li et al., (2011). 


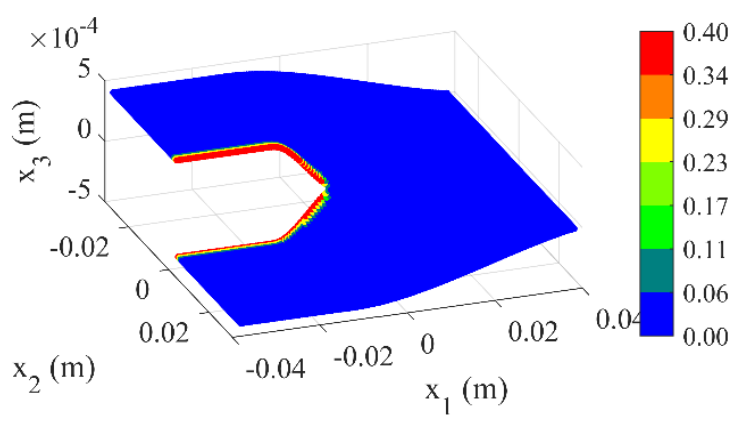

(a)

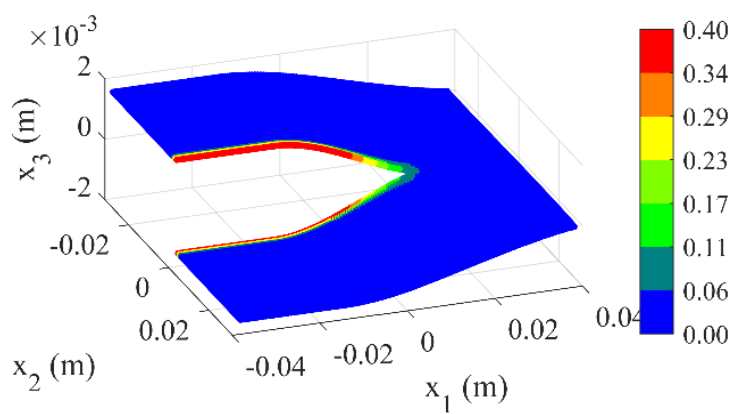

(c)

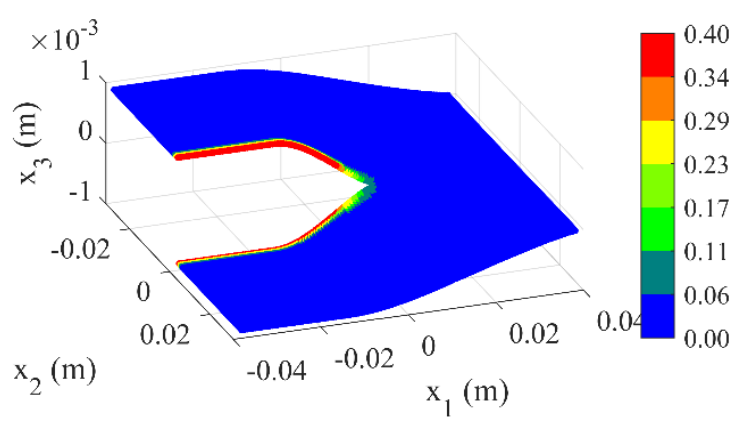

(b)

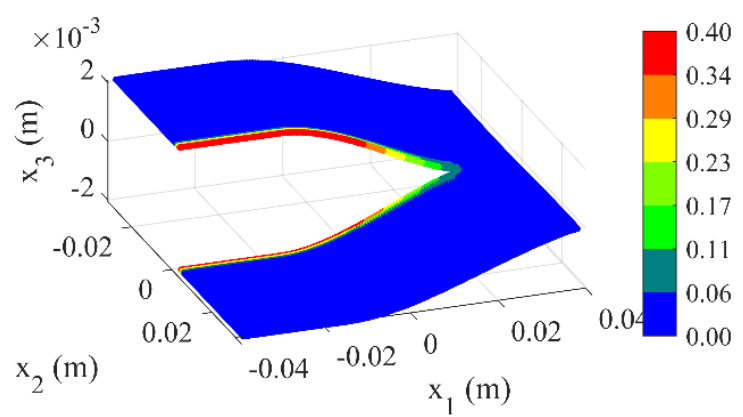

(d)

Fig. 6. Crack propagation when the applied vertical displacement (a) $u_{3}= \pm 3.9283 \times 10^{-4} \mathrm{~m}$ (b) $u_{3}= \pm 8.6454 \times 10^{-4} \mathrm{~m}$ (c) $u_{3}= \pm 0.0015 \mathrm{~m}$ (d) $u_{3}= \pm 0.0021 \mathrm{~m}$

5.2. Plate with a rectangular cut-out

A square plate with dimensions of $L=B=1 \mathrm{~m}$, and thickness of $h=0.01 \mathrm{~m}$ is investigated as shown in Fig. 7. The plate has a rectangular cut-out in the middle. The cut-out has dimensions of $l=0.4 \mathrm{~m}, b=0.3 \mathrm{~m}$.

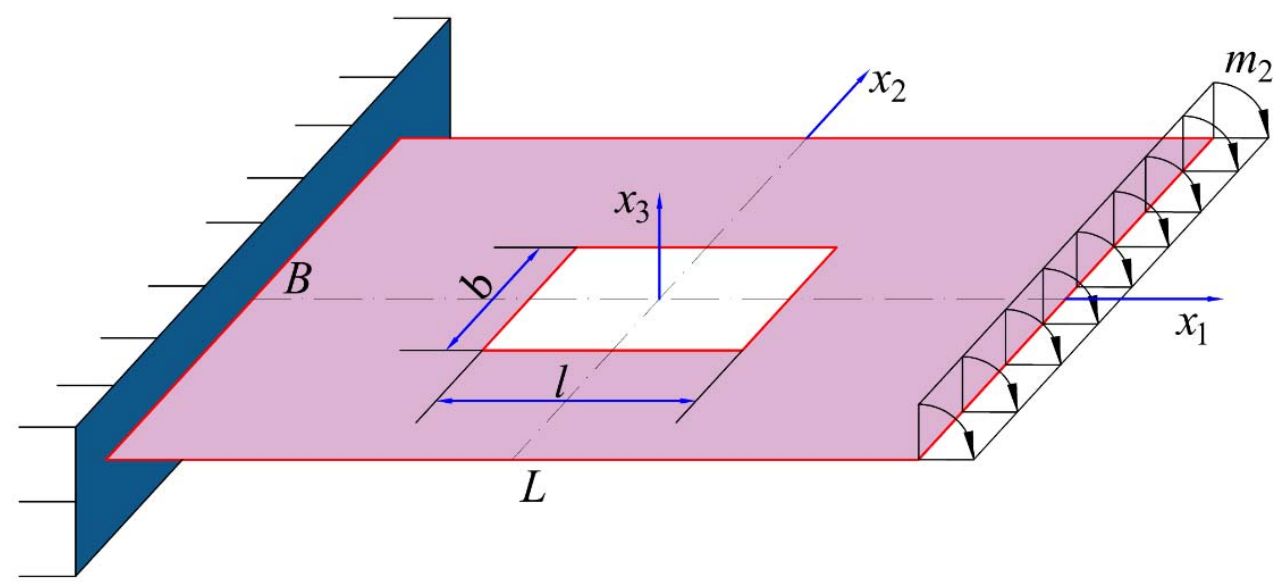

Fig. 7. Plate with a rectangular cut-out

The plate is made of steel with Young's modulus $E=2 \times 10^{11} \mathrm{~N} / \mathrm{m}^{2}$, Poisson's ratio $v=0.27$, the fracture toughness $K_{c}=70 \times 10^{6} \mathrm{Nm}^{-3 / 2}$ (Kalthoff, 1988) and the critical energy release 
rate $G_{c}=2.2714 \times 10^{4} \mathrm{~J} / \mathrm{m}^{2}$. The plate is fixed on the left edge and it is subjected to bending moments on the right edge as shown in Fig. 7.

In the PD model, the shell is uniformly discretized with a mesh size of $\Delta x=0.0067 \mathrm{~m}$ and the horizon size $\delta=3.015 \Delta x$ is used. In order to apply boundary conditions, three fictitious layers of material points are added on the left side of the plate and all degrees of freedom of these fictitious points and material points located at $x=-L / 2$ are set equal to zero (Nguyen and Oterkus, 2019b).

The right end of the plate is subjected to the bending moment per unit length $m_{2}$ as shown in Fig.7. Therefore, the loading for each material point on the right edge can be calculated as

$\mathbf{B}^{G}=\left[\begin{array}{llllll}0 & 0 & 0 & 0 & m_{2} \Delta x & 0\end{array}\right]^{T}$

At each load step, the value of the bending moment per unit length, $m_{2}$ is chosen in order to have at least one new broken interaction in the PD model. The value of $m_{2}$ is also not too large to avoid the breakages of too many bonds in a load step which may lead to numerical instabilities. The procedure for determining critical values of $m_{2}$ during the damage propagation process is presented in Appendix A.

Fig. 8 shows the damage evolution on the plate predicted by using the PD shell model. As shown in Fig. 8(a), at $20^{\text {th }}$ load step when the applied bending moment per unit length is $m_{2}=1.606 \times 10^{3} \mathrm{Nm} / \mathrm{m}$, the damage initiates at two corners of the cut-out, which are near the right edge of the plate where the bending moment is applied. In order to further investigate the behavior of the plate when it is subjected to the bending moment per unit length $m_{2}=1.606 \times 10^{3} \mathrm{Nm} / \mathrm{m}$ at $20^{\text {th }}$ load step, the variations of von Mises stresses captured by PD and FEA are compared as shown in Fig. 9. The FEA is conducted by using ANSYS SHELL181 element. The calculation of von Mises stresses in PD is described in Appendix B. As shown in Fig. 9, von Mises stress distribution in ANSYS is similar to PD results. Moreover, the maximum stress locations agree with the locations of the damage initiation predicted by PD as shown in Fig. 8(a).

The damages predicted by PD propagate nearly parallel to the right edge of the plate as shown in Figs. 8(b-d). After 1120 load steps when the applied bending moment is $m_{2}=1.066 \times 10^{3} \mathrm{Nm} / \mathrm{m}$, the damage propagation reaches to locations at $x_{2}= \pm 0.31 \mathrm{~m}$ as shown in Fig. 8(d). 


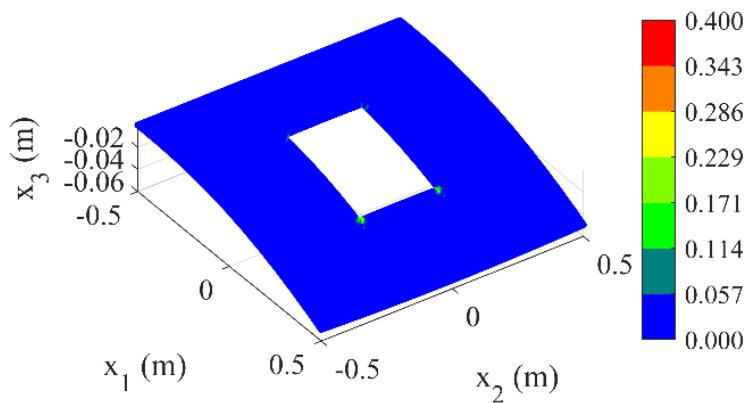

(a)

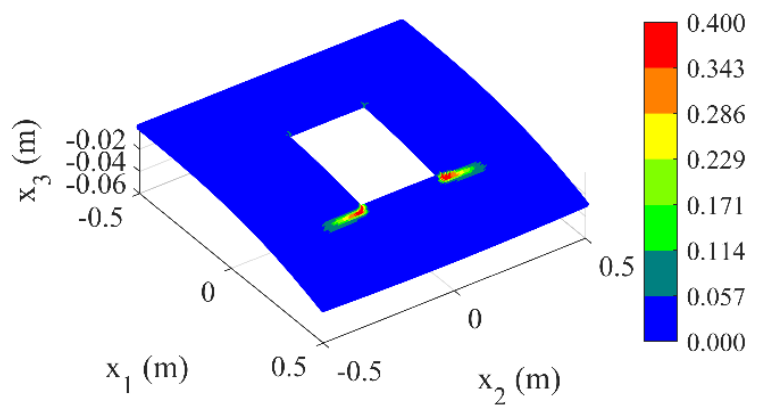

(c)

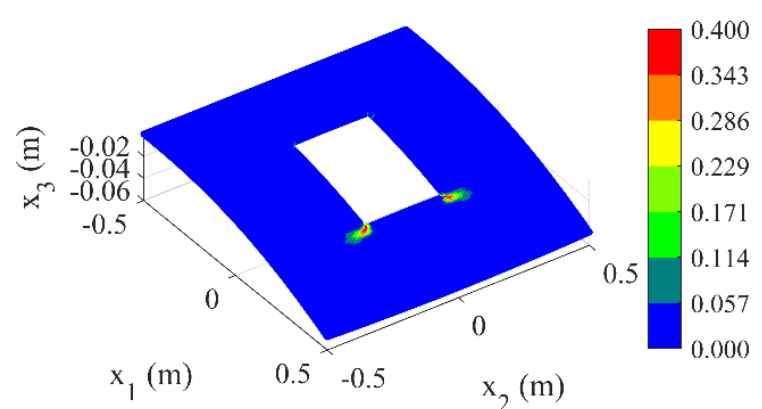

(b)

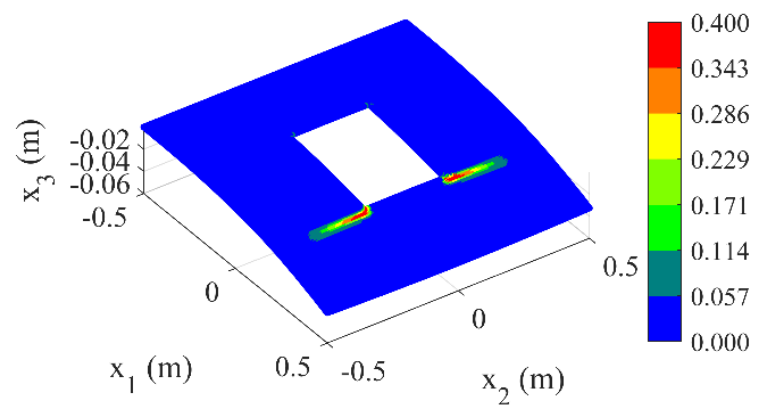

(d)

Fig. 8. Damage evolution on the plate at (a) $20^{\text {th }}$, (b) $400^{\text {th }}$, (c) $800^{\text {th }}$ (d) $1120^{\text {th }}$ load steps when applied bending moment $m_{2}=1.606 \times 10^{3}, 1.537 \times 10^{3}, 1.238 \times 10^{3}, 1.066 \times 10^{3} \mathrm{Nm} / \mathrm{m}$, respectively

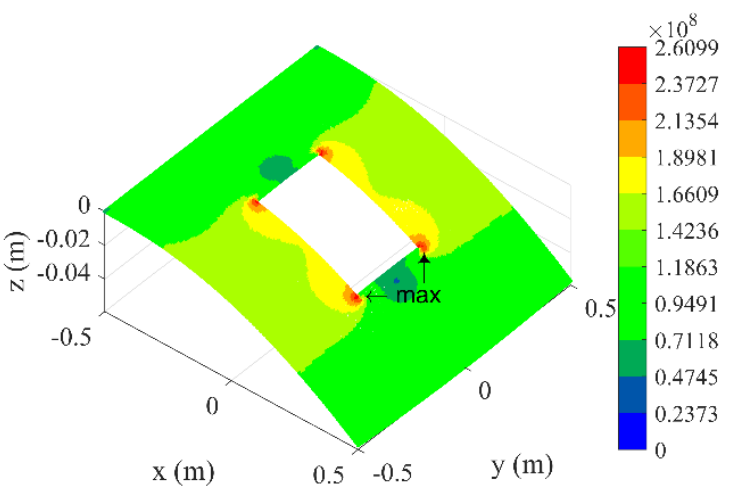

(a)

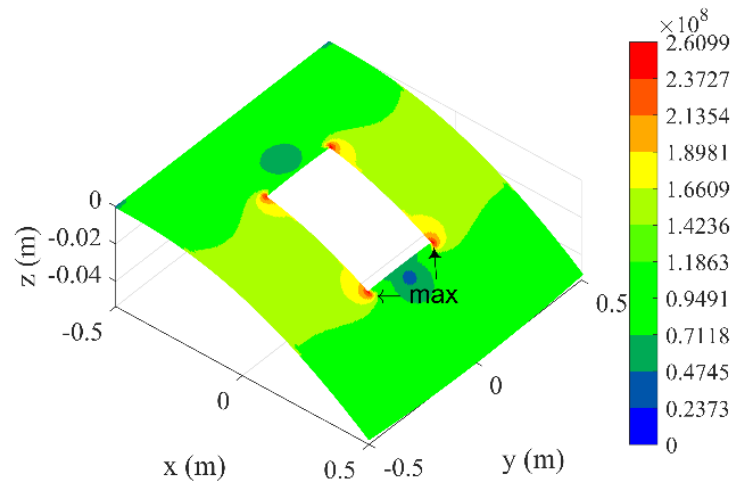

(b)

Fig. 9. Variation of von Mises stress (Pa) predicted by (a) PD, (b) FEA

Fig. 10 shows the loading history of applied bending moment per unit length, $m_{2}$ and corresponding crack paths, shown in black, on the plate at different load steps. Since the plate is symmetric with respect to the $x_{1}$ axis, only the crack path on the positive direction of the $x_{2}$ 
axis is presented. As can be seen from the figure, as the crack propagates during the first 200 load steps, the applied bending moment, $m_{2}$ reduces quickly from $1.606 \times 10^{3} \mathrm{Nm} / \mathrm{m}$ to $1.474 \times 10^{3} \mathrm{Nm} / \mathrm{m}$. Initially, the cracks propagate with an angle of $10^{\circ}$ with respect to the $x_{1}$ axis. Later, the cracks change their directions and propagate parallel to the right edge of the plate. At $400^{\text {th }}$ load step, the applied bending moment increases slightly to $1.537 \times 10^{3} \mathrm{Nm} / \mathrm{m}$ . Afterward, the cracks propagate towards the location $\left(x_{1}=0.2133 \mathrm{~m}, x_{2}= \pm 0.31 \mathrm{~m}\right)$ at the $1120^{\text {th }}$ load step. The required bending moment is sharply reduced due to reduced strength caused by damage growth.

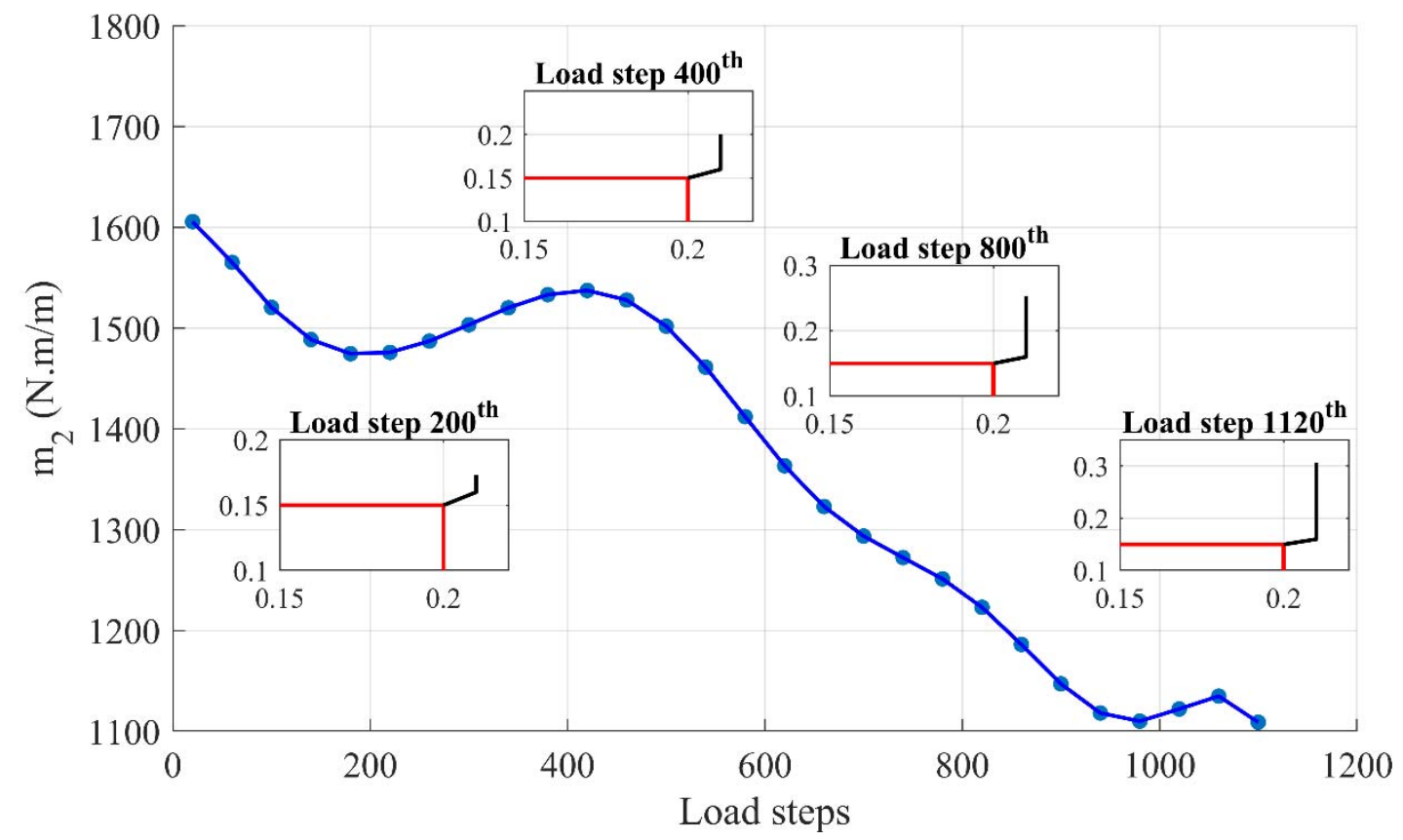

Fig. 10. Load history of applied bending moment per unit length, $m_{2}$

\subsection{Damage prediction for a ship structure}

An experimental MST4-ship model developed by Nishihara (1984) with dimensions of $L \times B \times H=0.9 \times 0.72 \times 0.72 \mathrm{~m}^{3}$ is investigated. The $3 \mathrm{D}$ model and the cross-section details of the ship are shown in Fig. 11.

The ship is made of steel with Young's modulus $E=2.08 \times 10^{11} \mathrm{~N} / \mathrm{m}^{2}$, Poisson's ratio $v=0.281$ (Nishihara, 1984), fracture toughness, $K_{c}=54 \times 10^{6} \mathrm{Nm}^{-3 / 2}$ (Matthews, 1973), and energy release rate, $G_{c}=1.2912 \times 10^{4} \mathrm{~J} / \mathrm{m}^{2}$.

The ship is investigated for two basic loading conditions which are bending and torsion as shown in Fig. 12. In the PD model, the ship is discretized with a mesh size of $\Delta x=0.02 \mathrm{~m}$. Similar to the previous examples, the horizon size $\delta=3.015 \Delta x$ is used. In order to apply loading conditions, two thick rigid plates are added at two ends of the model as shown in Fig. 13. The rigid plates are represented by Young's modulus $E_{r}=20 \times E$, Poisson's ratio $v_{r}=v$, and thickness $h_{r}=20 \times h$. 


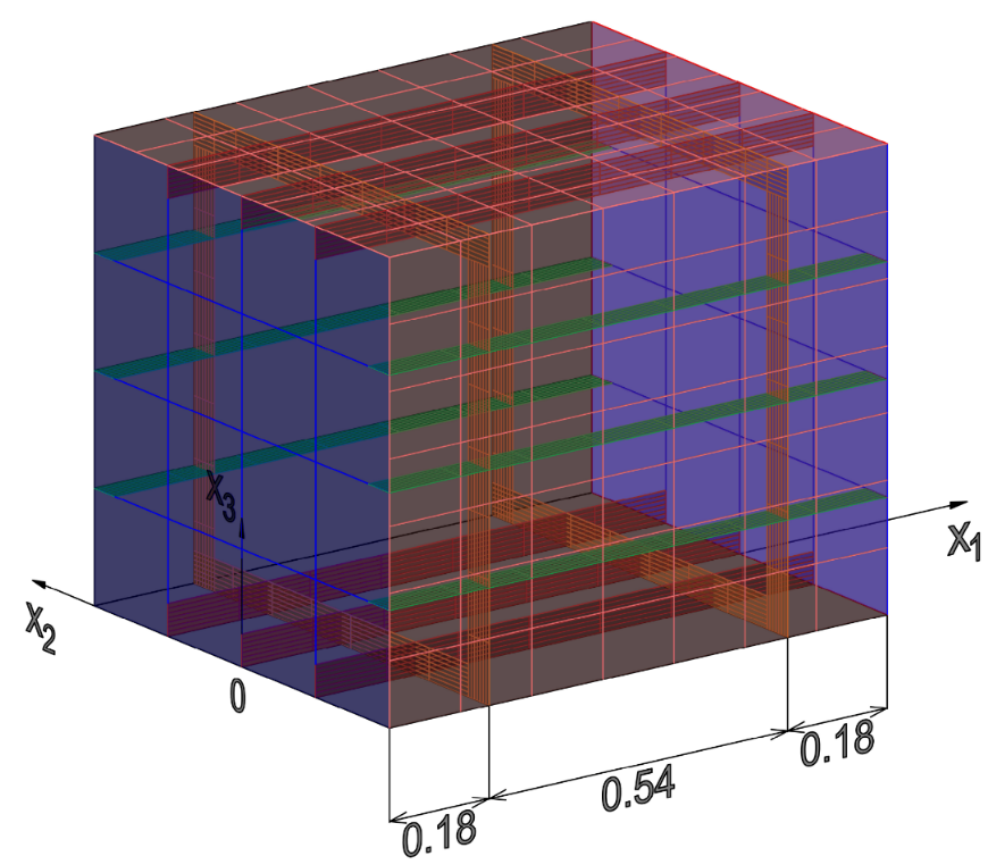

(a)

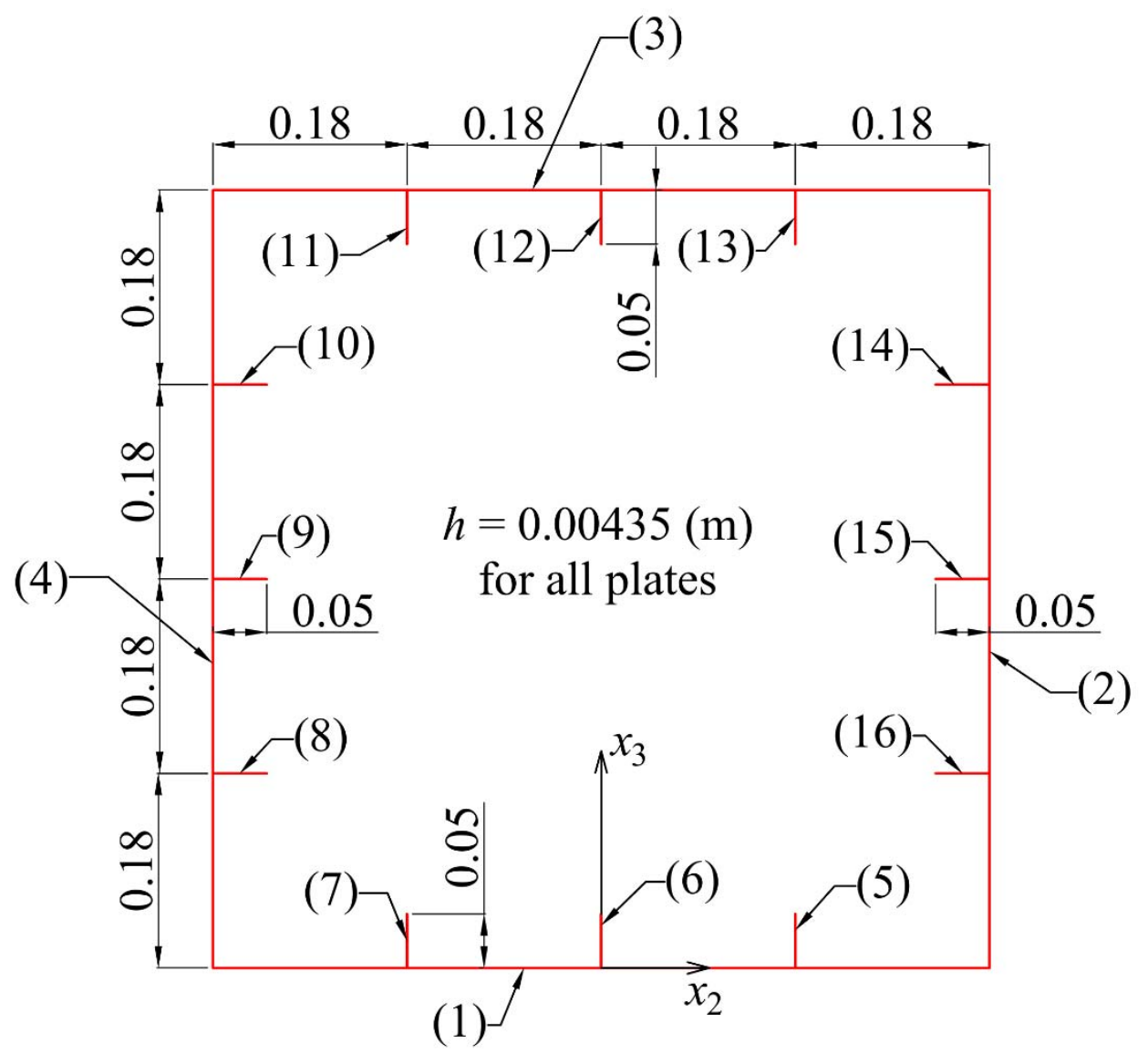

(b)

Fig. 11. MST4 ship model (Nishihara, 1984) (a): 3D model (b): ship cross-section at $x_{1}=0.45 \mathrm{~m}$ 


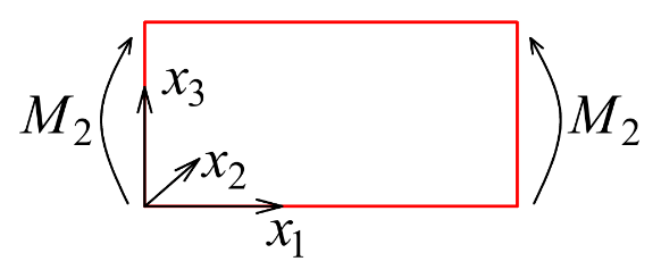

(a)

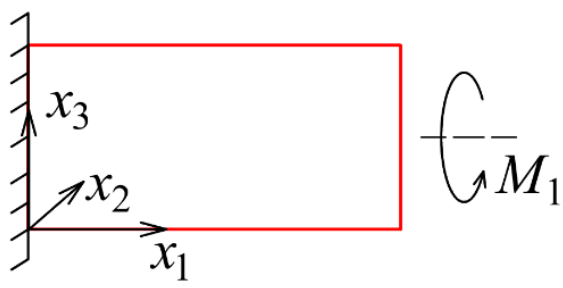

(b)

Fig. 12. Three basic loading conditions (a): Sagging, (b): Torsion

In Fig. 13, the plates on the deck, the sides and the bottom are discretized with 1794 material points. Each longitudinal frame includes 184 material points. Each transverse frame includes 560 material points. Moreover, each rigid plate is discretized into 1521 material points. Therefore, there are totally 12058 material points corresponding to 72348 DOFs used for the intact ship model.

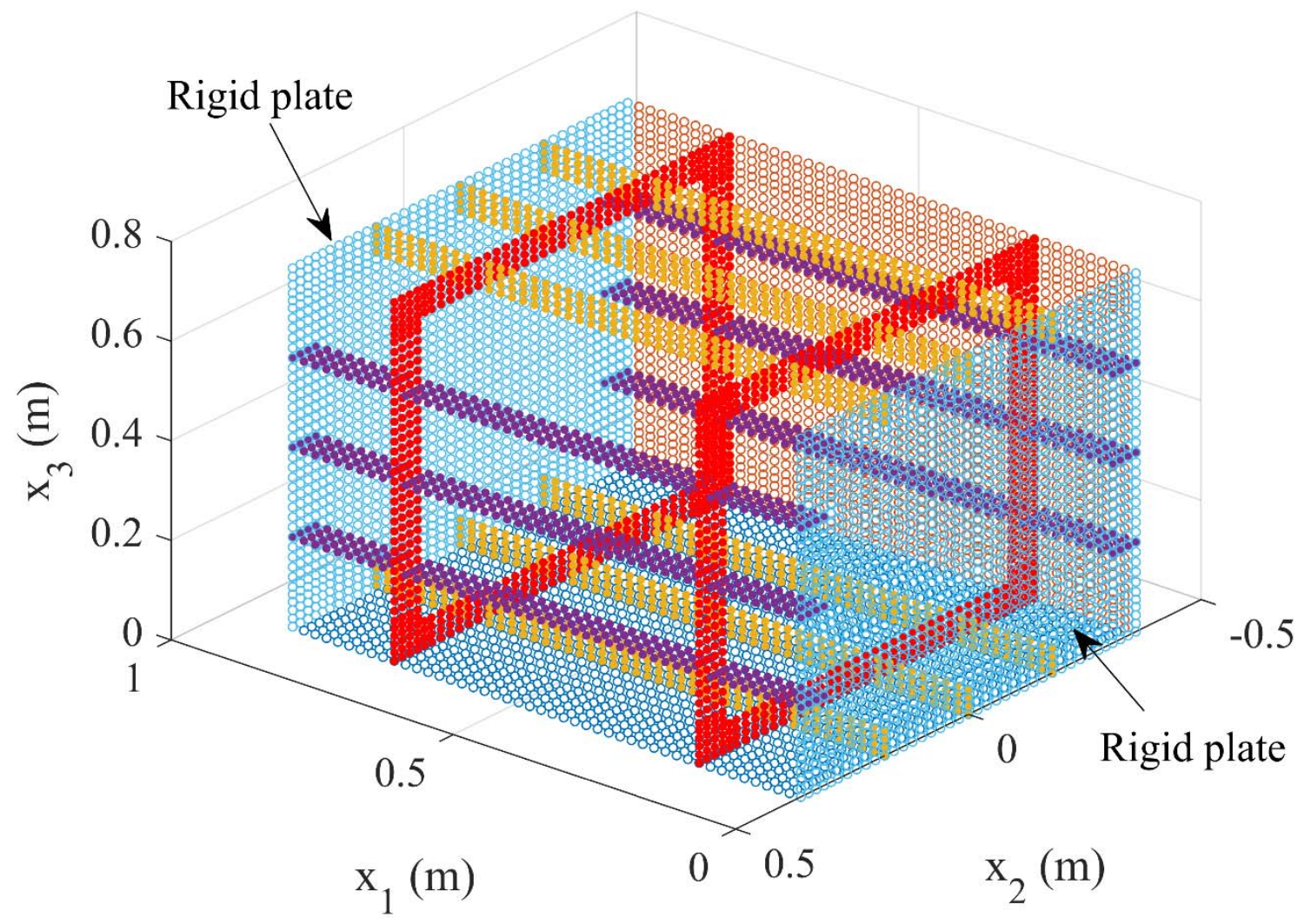

Fig. 13. PD discretized model for the ship model (the plates at $x_{3}=H$ and $x_{2}=B / 2$ are hidden for visualization purposes)

\subsubsection{Ship structure subjected to bending (sagging)}

In this section, the ship is subjected to bending loading as shown in Fig. 12(a). The bending moments for the material points located at the left end of the model at $x_{1}=0$ are applied as

$M_{2 L}=M_{2} / N_{L}$

On the other hand, the bending moments for the material points located at the right end of the model at $x_{1}=L$ are applied as

$M_{2 R}=-M_{2} / N_{R}$ 
where $N_{L}$ and $N_{R}$ represent total numbers of material points located at the left end and right end of the model, respectively. The parameter $M_{2}$ represents the total bending moment applied on the ship structure as shown in Fig.12(a).

Similar to the previous example, the value of bending moment, $M_{2}$, at each load step is chosen as the critical value in order to have at least one new broken interaction. The procedure to determine the critical value for $M_{2}$ during the damage propagation process at each load step is presented in Appendix A.

The boundary conditions for this loading condition can be described as

$$
\begin{aligned}
& u_{1}=0 \text { at }\left(x_{1}=L / 2,-B / 2 \leq x_{2} \leq B / 2,0 \leq x_{3} \leq H\right) \\
& u_{2}=0 \text { at }\left(x_{1}=0, x_{2}=0,0 \leq x_{3} \leq H\right) \\
& u_{2}=0 \text { at }\left(x_{1}=L, x_{2}=0,0 \leq x_{3} \leq H\right) \\
& u_{3}=0 \text { at }\left(x_{1}=0,-B / 2 \leq x_{2} \leq B / 2, x_{3}=H\right) \\
& u_{3}=0 \text { at }\left(x_{1}=L,-B / 2 \leq x_{2} \leq B / 2, x_{3}=H\right)
\end{aligned}
$$

The ship structure is investigated for different scenarios. First, the intact ship subjected to bending loading is investigated. Next, the ship is assumed to have square cut-outs on the bottom with dimensions of $0.2 \times 0.2 \mathrm{~m}^{2}$ or $0.4 \times 0.4 \mathrm{~m}^{2}$ as shown in Fig. 14 .

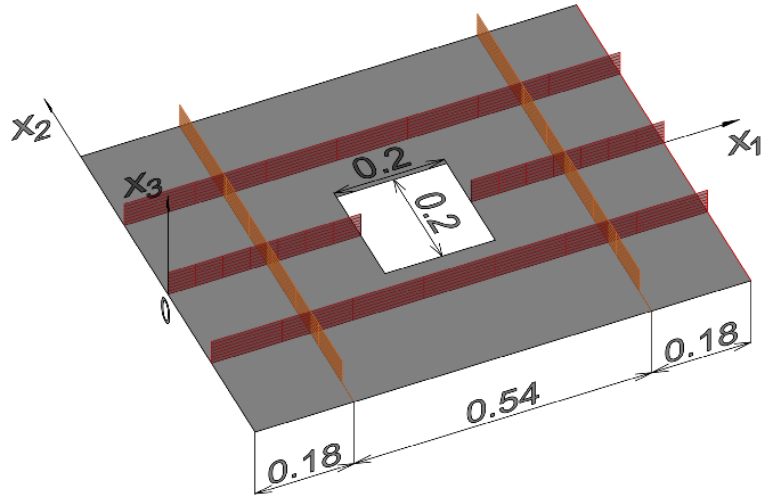

(a)

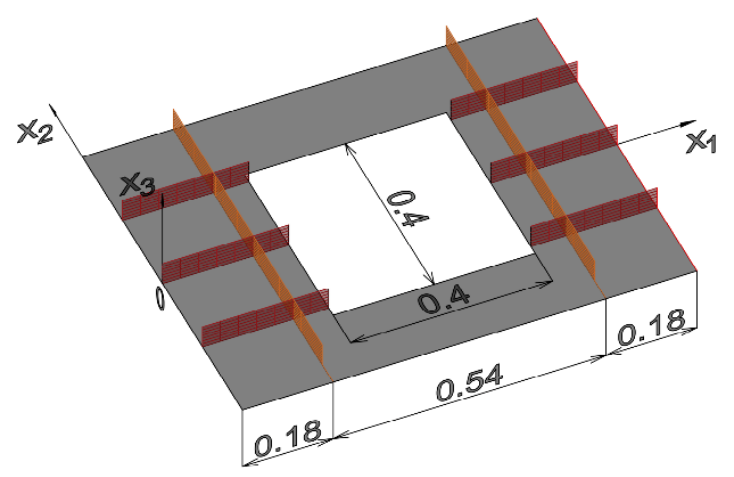

(b)

Fig. 14. The bottom of the ship with a square cut-out with dimensions of (a) $0.2 \times 0.2 \mathrm{~m}^{2}$, (b)

$$
0.4 \times 0.4 \mathrm{~m}^{2}
$$

\section{Intact ship subjected to bending loading}

By using the uniform mesh size of $\Delta x=0.02 \mathrm{~m}$, there are totally 12058 material points corresponding to 72348 degrees of freedom in the PD discretized model. Figs. 15-17 present the damage evolution on the ship structure. As shown in Fig. 15, at $400^{\text {th }}$ load step when the applied bending moment is $M_{2}=4.73 \times 10^{5} \mathrm{~N} . \mathrm{m}$, there is significant damage in the middle of the deck. As the applied bending continues, the damage on the deck propagates toward two sides of the ship as shown in Figs. 16-17. At $1200^{\text {th }}$ load step when the applied bending moment is $M_{2}=2.05 \times 10^{5} \mathrm{~N} . \mathrm{m}$, the damage develops beyond the ship deck and propagates towards the ship side as shown in Fig. 17. 


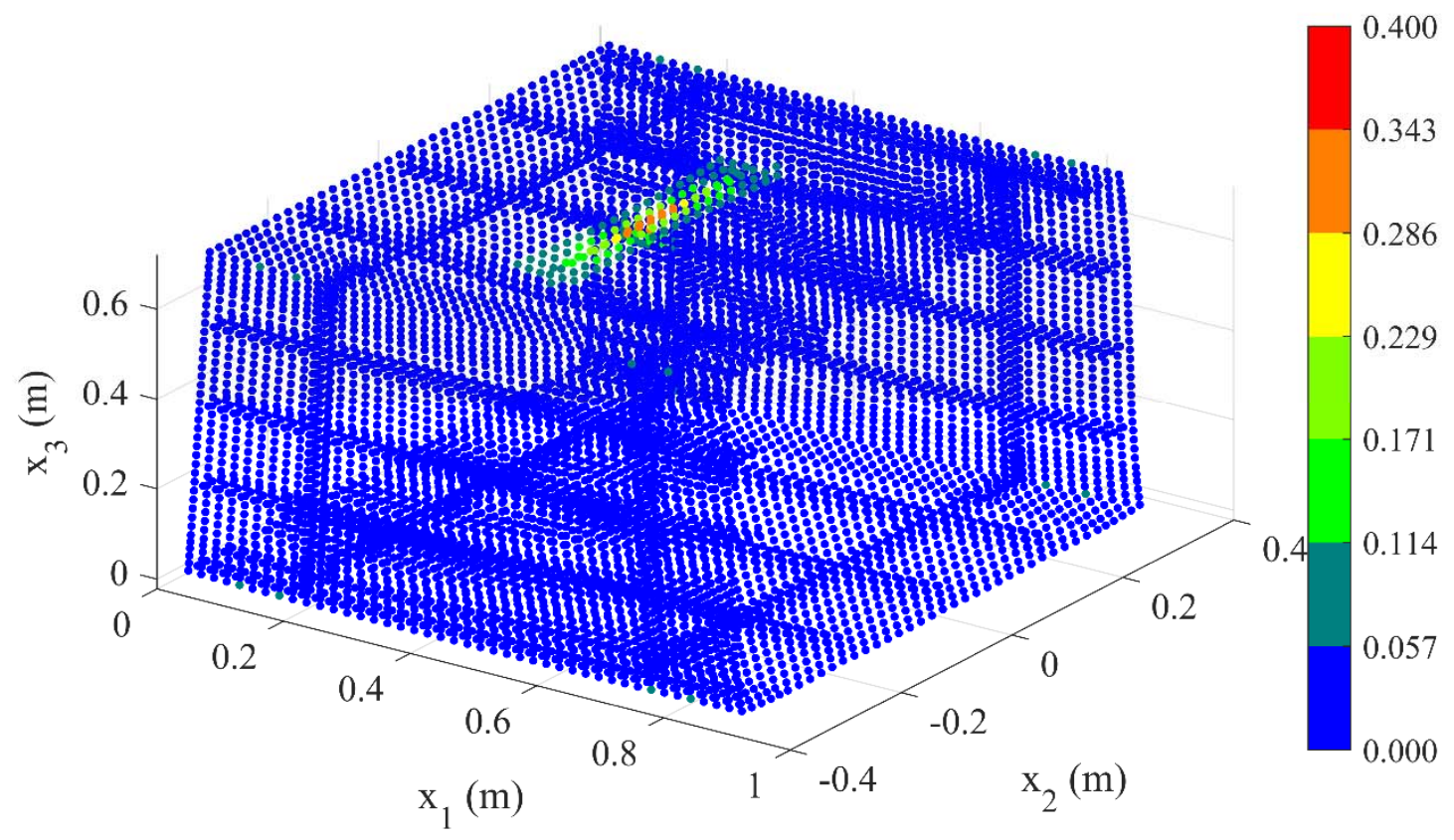

Fig. 15. Damage plot at load step $400^{\text {th }}$ when applied bending moment $M_{2}=4.73 \times 10^{5}$ N.m (displacements are magnified 100 times for deformed configuration. Rigid plates are removed for visualization.)

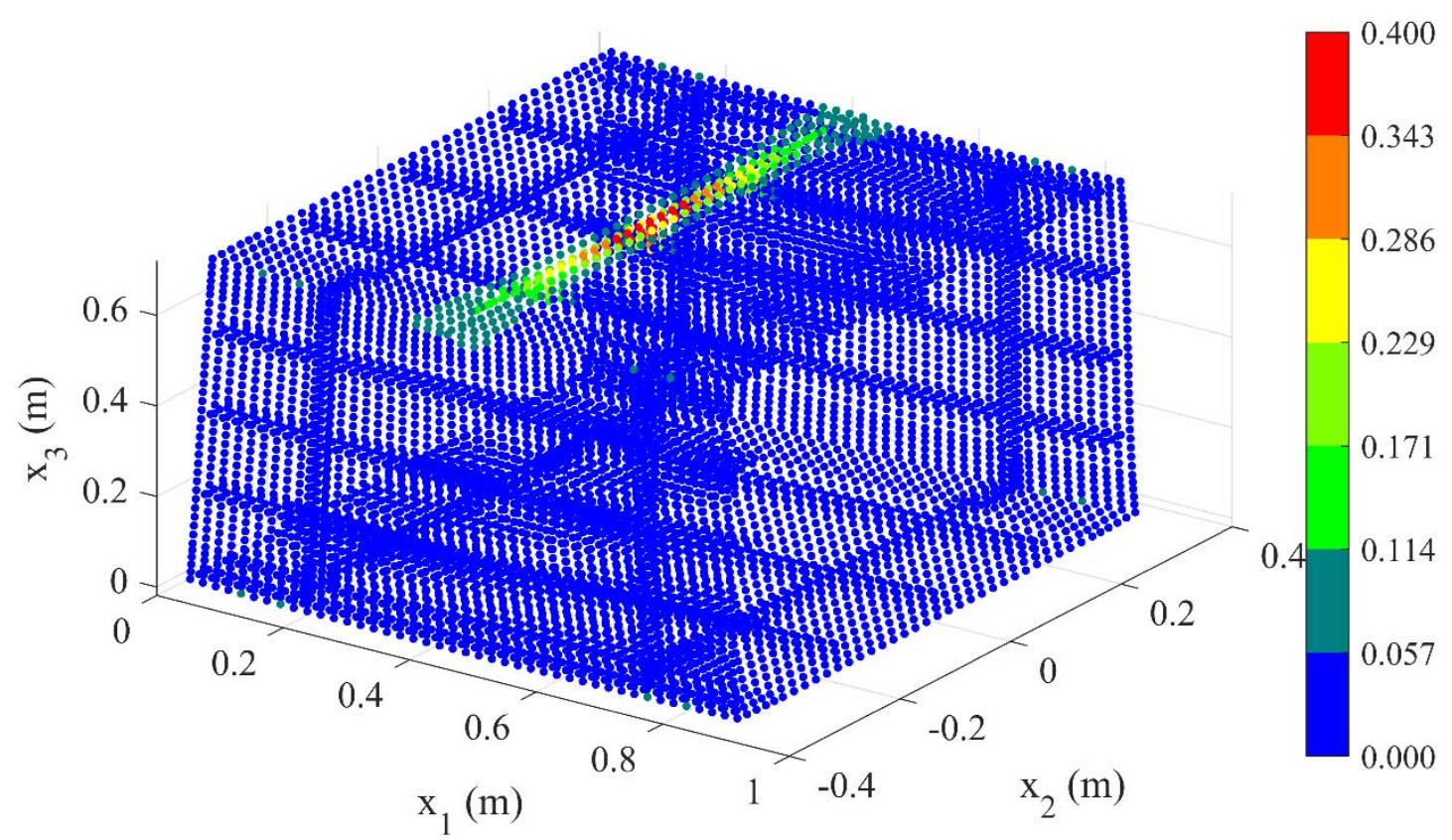

Fig. 16. Damage plot at $800^{\text {th }}$ load step when applied bending moment $M_{2}=3.19 \times 10^{5}$ N.m (displacements are magnified 100 times for deformed configuration. Rigid plates are removed for visualization.) 


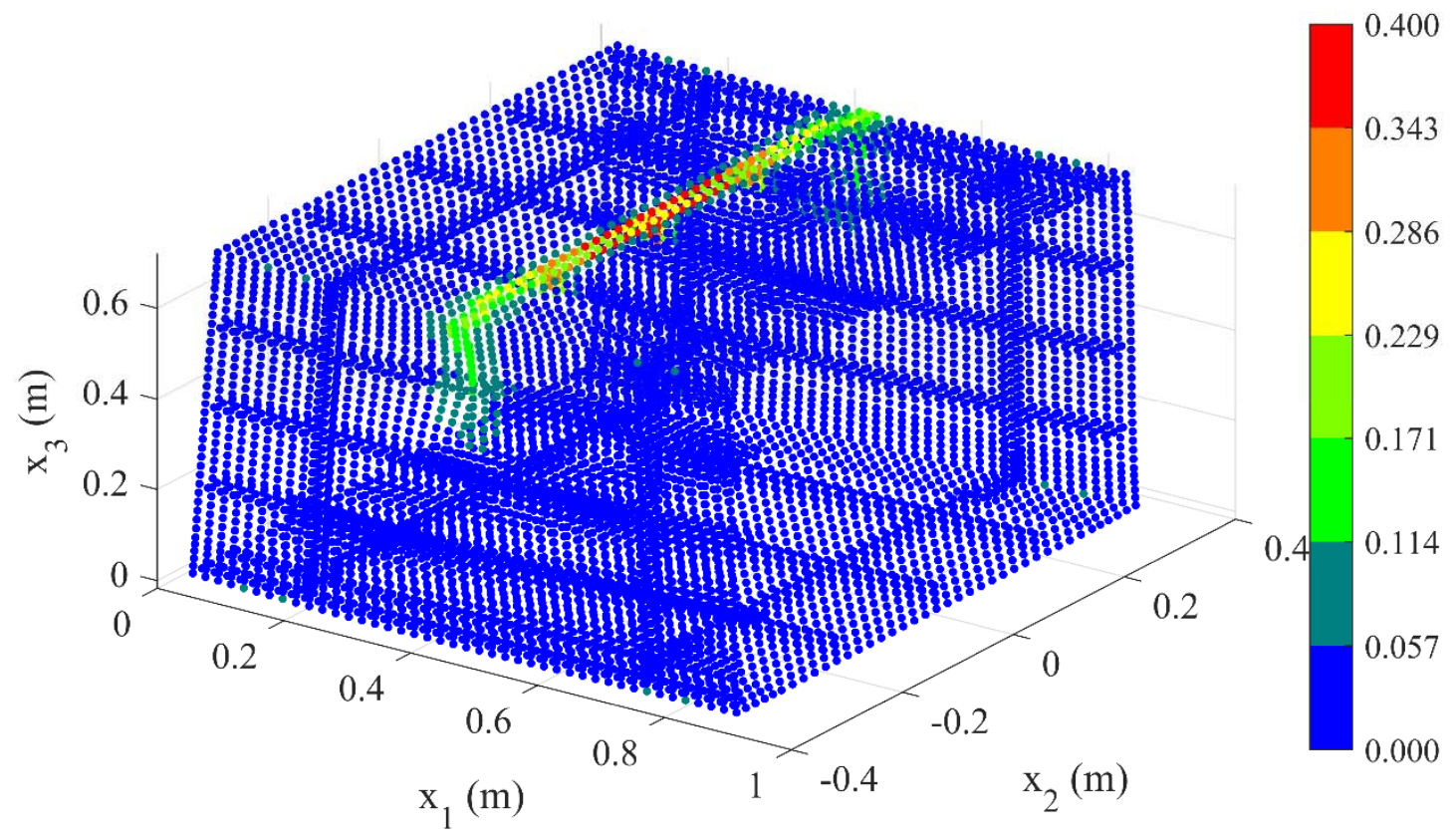

Fig. 17. Damage plot at $1200^{\text {th }}$ load step when applied bending moment $M_{2}=2.05 \times 10^{5}$ N.m (displacements are magnified 100 times for deformed configuration. Rigid plates are removed for visualization.)

In order to visualize the damage process on the structure, the damage evolutions on the middle sections of the ship are shown in Fig. 18. For visualization purposes, the structure is shown in the undeformed configuration and the range of damage coefficient is reduced to $0 \leq \phi \leq 0.2$. As it can be seen from the figures, the damage initiates at the middle location on the ship deck after 200 load steps as shown in Fig. 18(b). Later, the damage propagates horizontally to ship sides, while the longitudinal shells are also damaged as shown in Figs. 18(c-f). After 1200 load steps, the structures on the ship deck are completely damaged and the damage starts growing vertically on the ship sides.

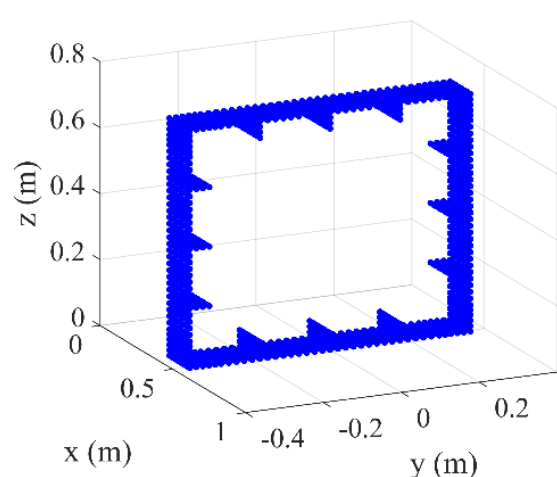

(a)

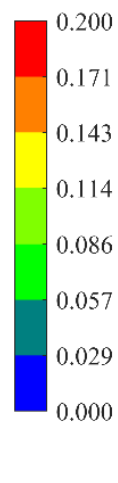

$\mathrm{x}(\mathrm{m})$

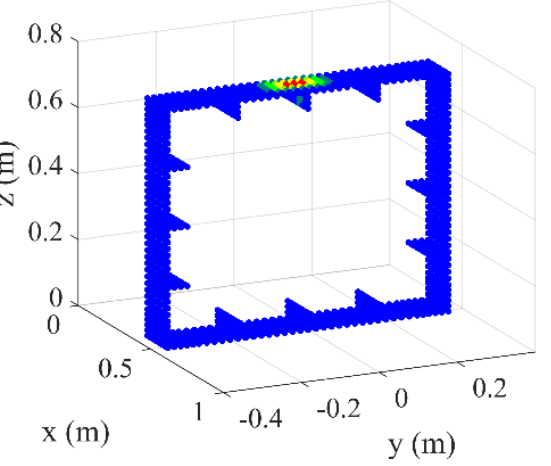

(b)

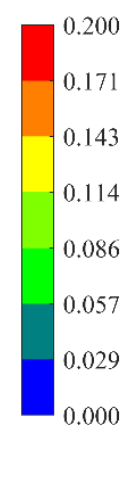




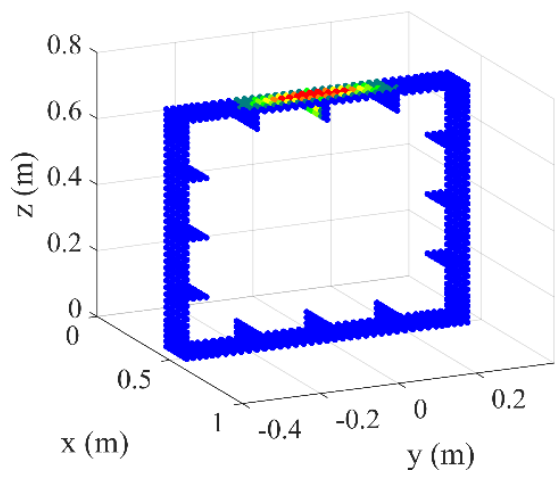

(c)

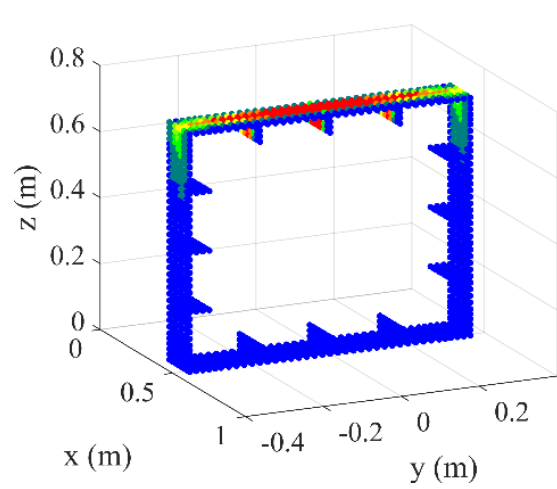

(e)
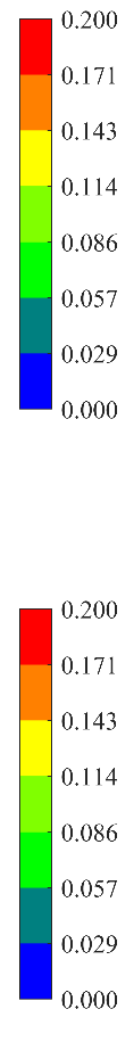

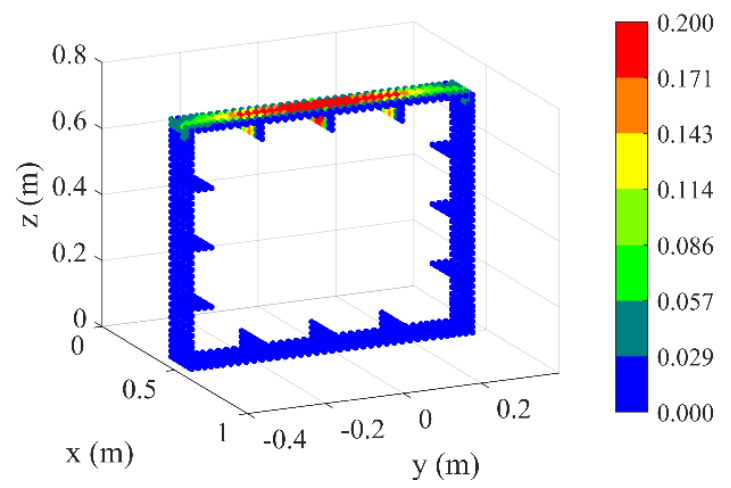

(d)

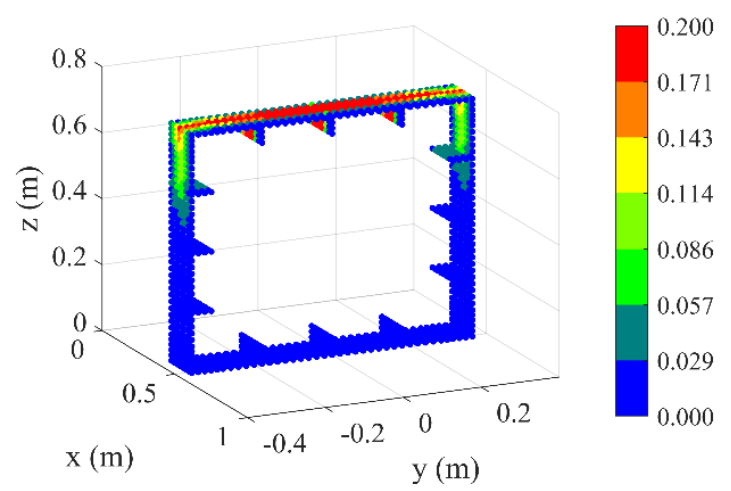

(f)

Fig. 18. Damage plot for the middle parts load step at (a) $20^{\text {th }}$, (b) $200^{\text {th }}$, (c) $400^{\text {th }}$, (d) $800^{\text {th }}$, (e) $1000^{\text {th }}$, (f) $1200^{\text {th }}$ load step. (The figures are shown in the undeformed configuration)

\section{Ship with square cut-out subjected to bending loading}

Similar to the previous case, the mesh size of $\Delta x=0.02 \mathrm{~m}$ is used. Therefore, in the PD discretized model for the ship with the smaller cut-out, there are totally 11904 material points corresponding to 71424 degrees of freedom. In the PD discretized model for the ship with the larger cut-out, there are 11428 material points and 68568 degrees of freedom. Figs. 19-21 present damage evolution on the ship with $0.2 \times 0.2 \mathrm{~m}^{2}$ cut-out. In this case, the damages initiate at four corners of the cut-out as shown in Fig. 19. As can be seen from the figures, the cracks propagate from four corners of the cut-out towards the two sides of the ship structure. As the load is continuously applied, the cracks propagate vertically on two sides of the ship structure as shown in Figs. 20-21. 


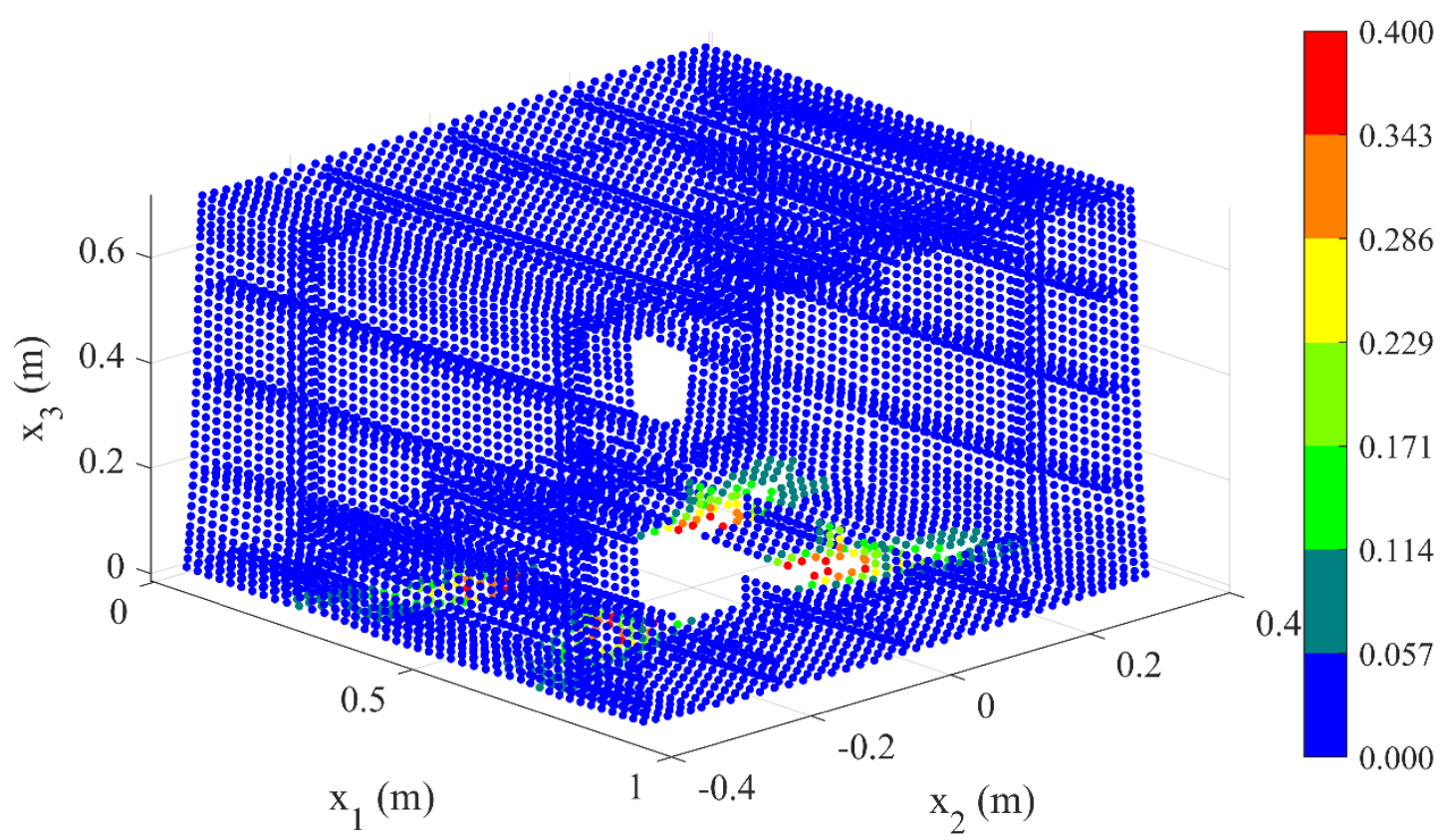

(a)

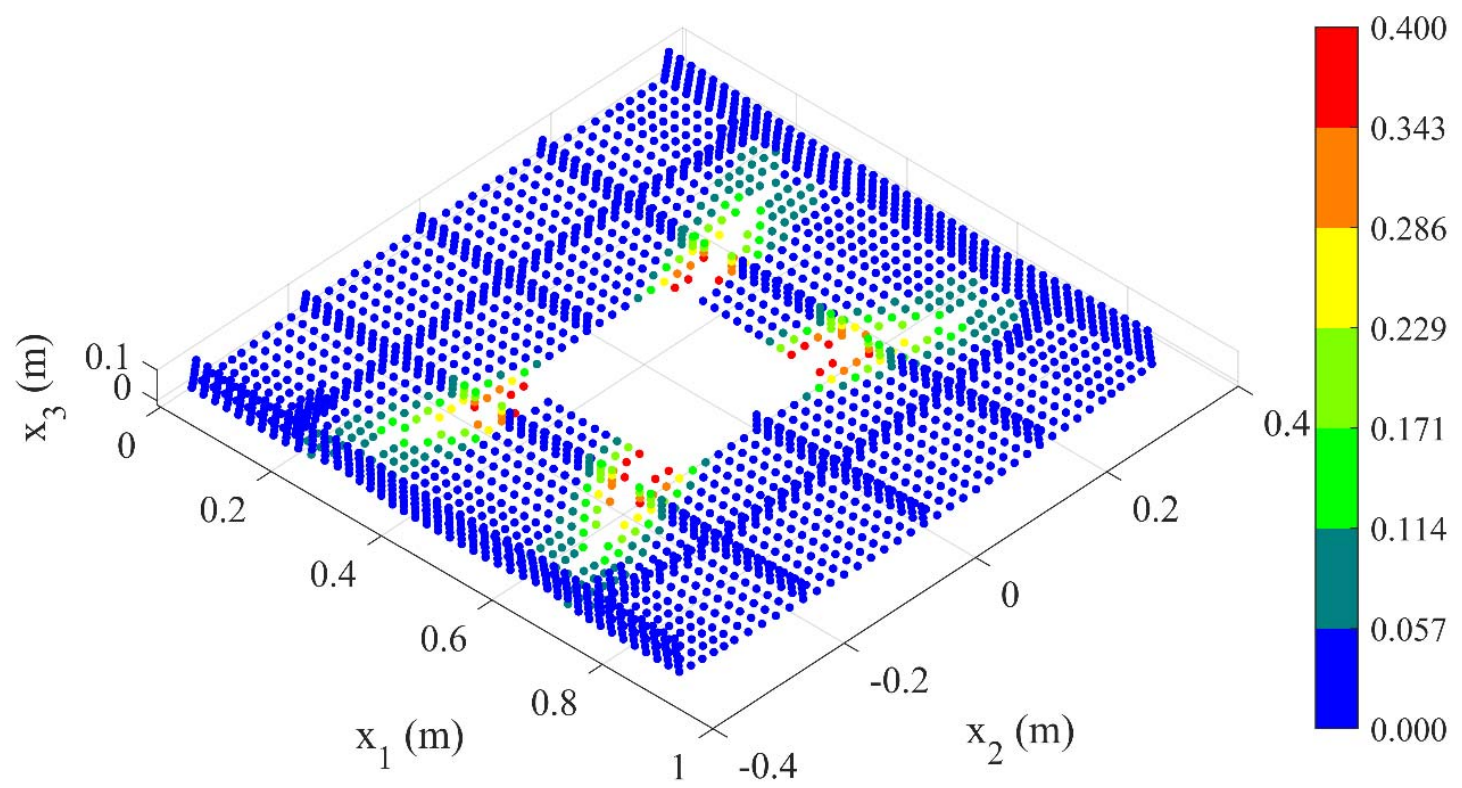

(b)

Fig. 19. Damage plot at load step $400^{\text {th }}$ when applied bending moment $M_{2}=2.22 \times 10^{5}$ N.m

(a): 3D model (b): a view for material points with $x_{3} \leq 0.1 \mathrm{~m}$ (displacements are magnified 100 times for deformed configuration. Rigid plates are removed for visualization.) 


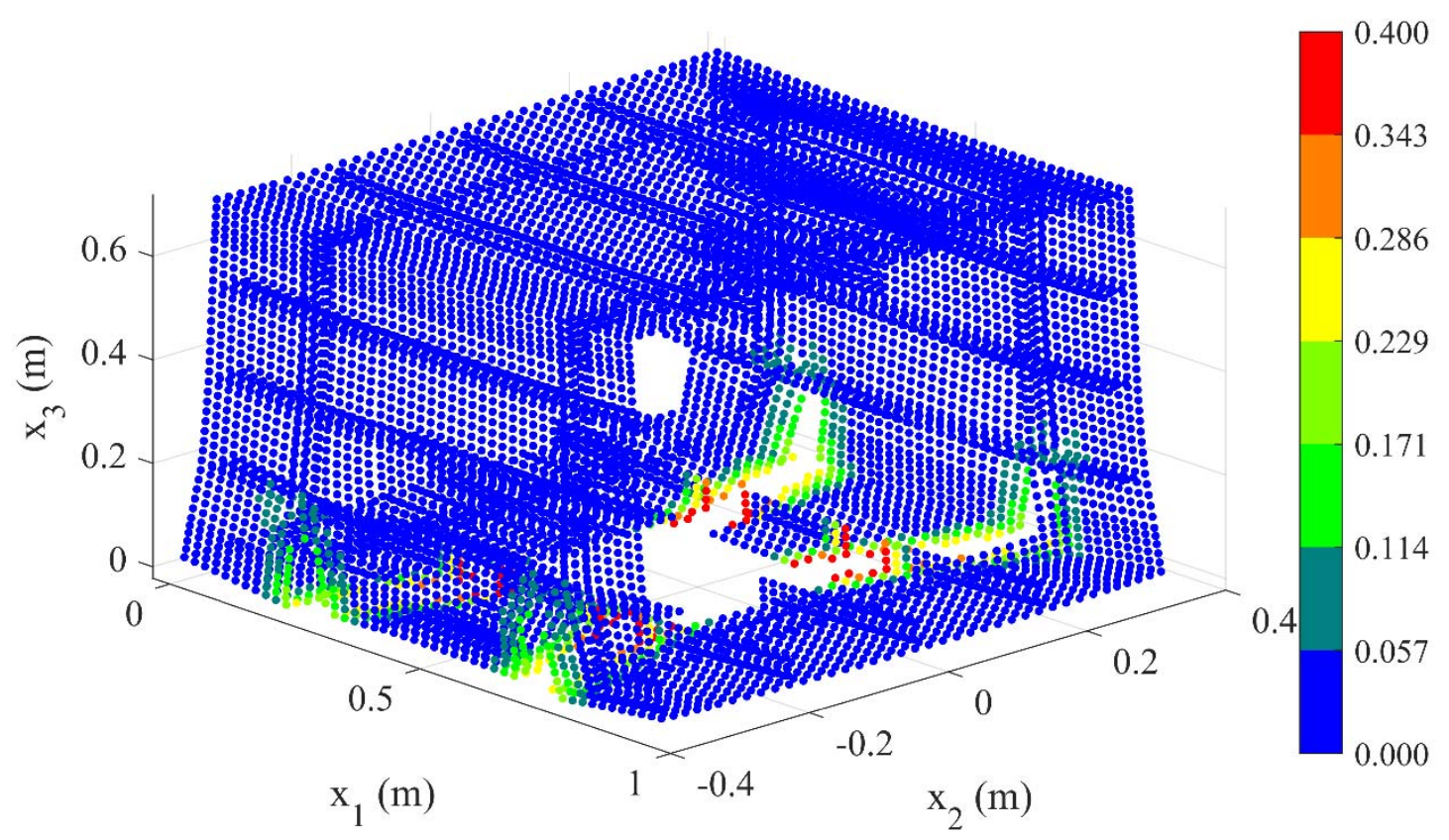

(a)

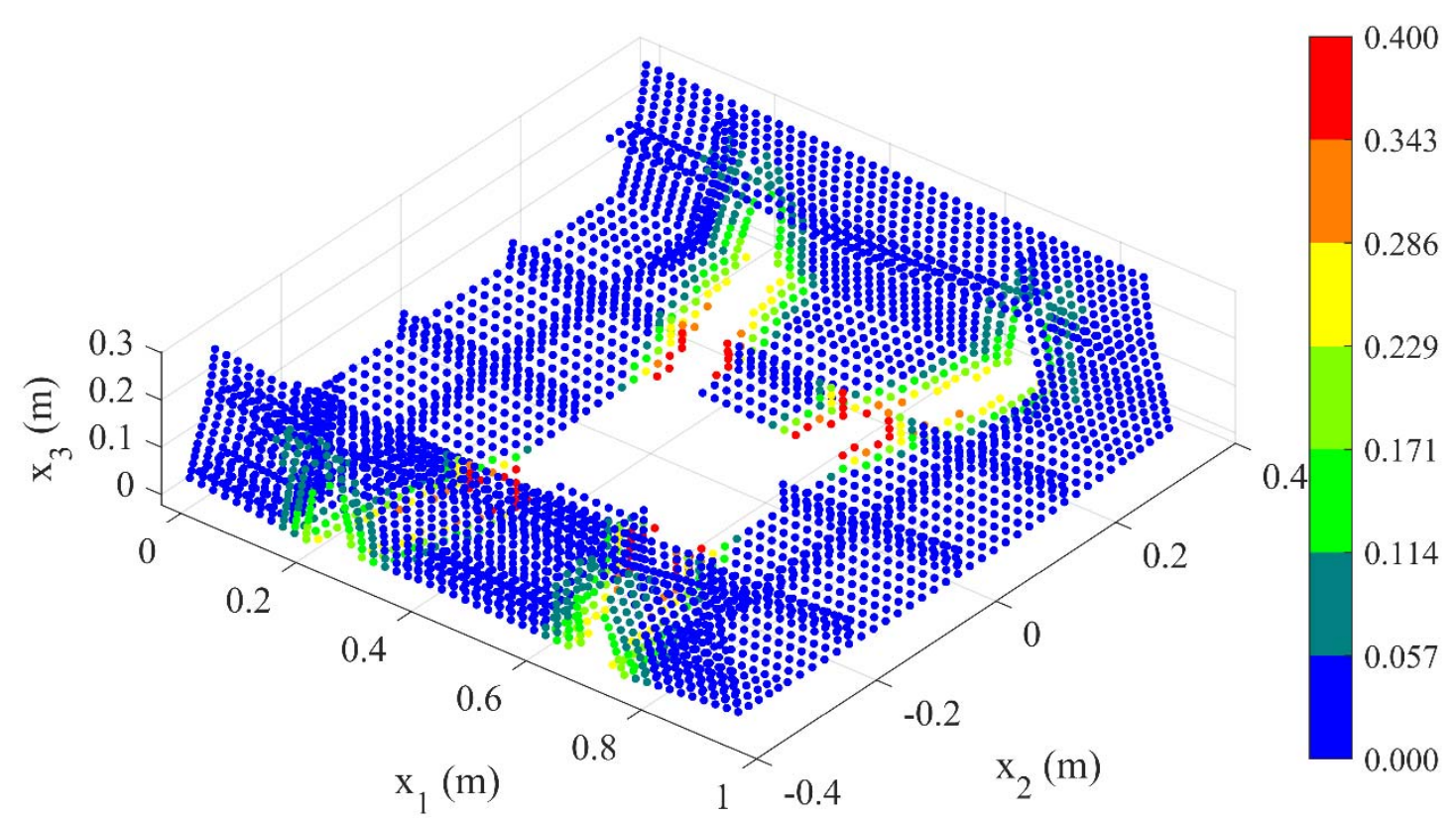

(b)

Fig. 20. Damage plot at $800^{\text {th }}$ load step when applied bending moment $M_{2}=1.58 \times 10^{5}$ N.m (a): 3D model (b): a view for material points with $x_{3} \leq 0.3 \mathrm{~m}$ (displacements are magnified 100 times for deformed configuration. Rigid plates are removed for visualization.) 


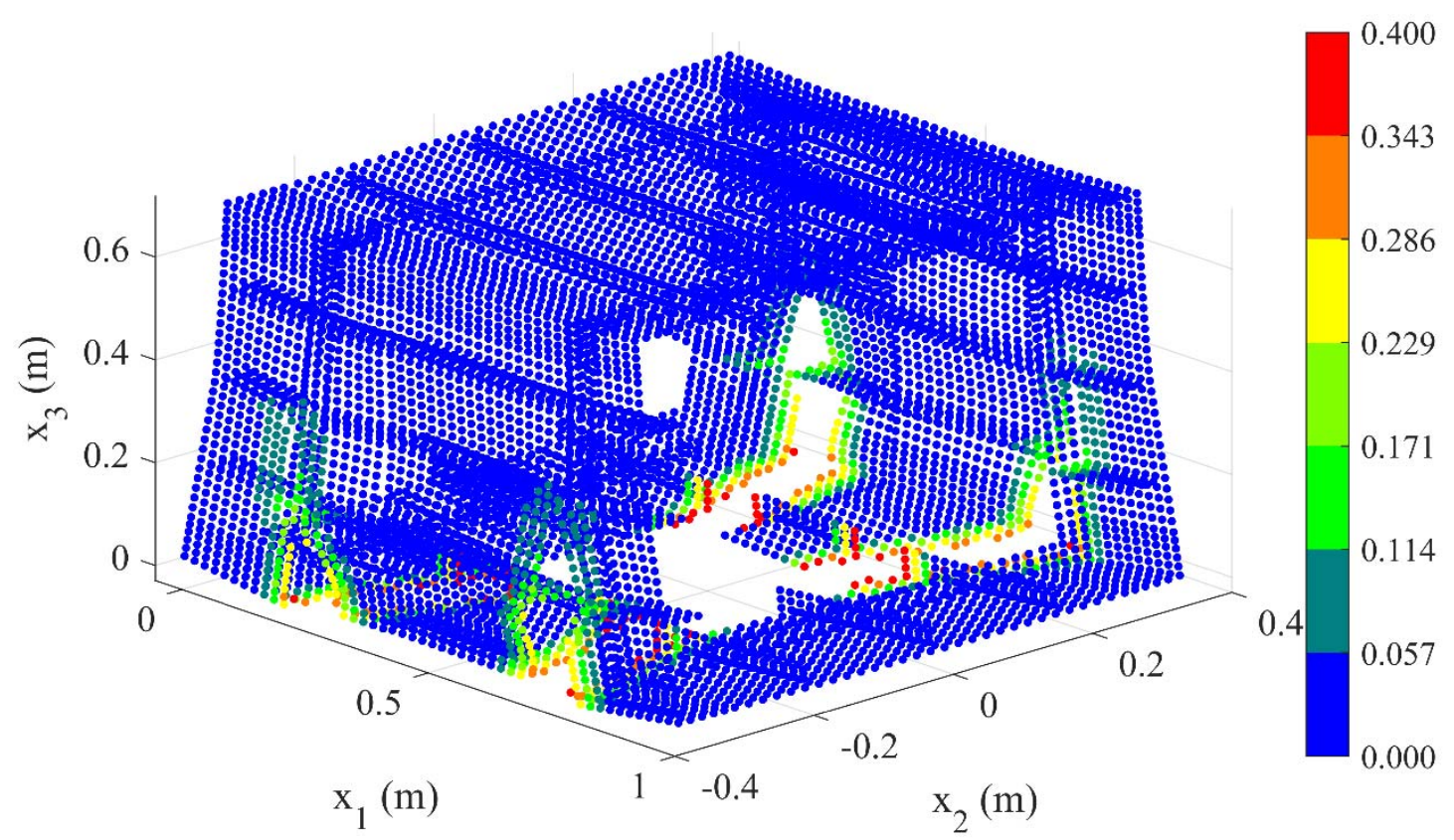

(a)

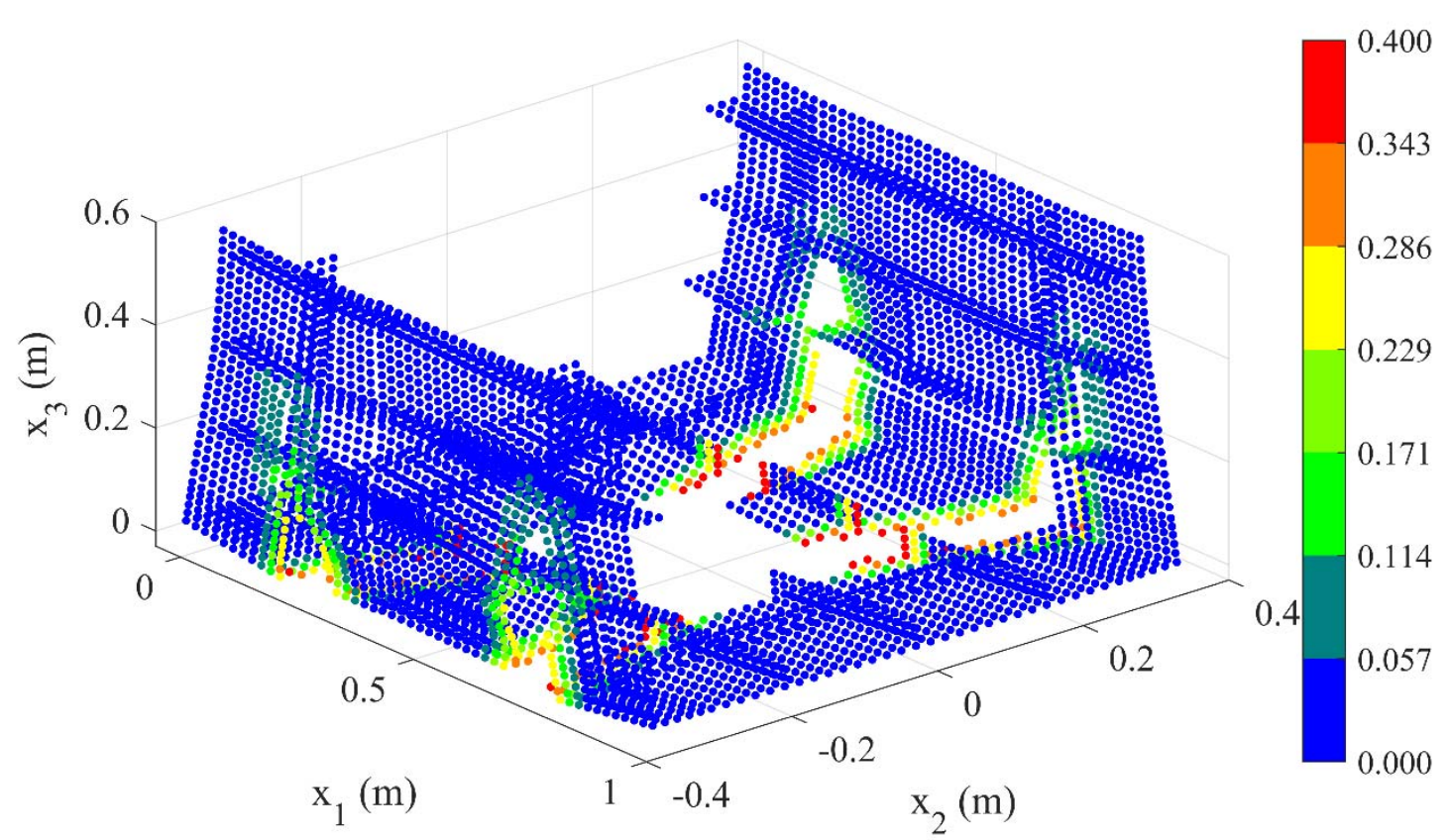

(b)

Fig. 21. Final damage plot on the ship at $1200^{\text {th }}$ load step when applied bending moment $M_{2}=8.53 \times 10^{4}$ N.m (a): $3 \mathrm{D}$ model (b): a view for material points with $x_{3} \leq 0.6 \mathrm{~m}$ (displacements are magnified 100 times for deformed configuration. Rigid plates are removed for visualization.)

\section{Variations of the critical bending moment versus the change in the moment of inertia}

Fig. 22 shows the variations of bending moment, $M_{2}$ and change in the moment of inertia, $\Delta I_{2}$ , of the ship cross-section for three cases. The moment of inertia reduces with respect to the intact ship. The reduction of the moment of inertia, $\Delta I_{2}$, can be defined as 


$$
\Delta I_{2}=\frac{I_{2 \text { (current) }}-I_{2 \text { (intact) }}}{I_{2 \text { (intact) }}} \times 100 \%
$$

where $I_{2 \text { (intact) }}$ and $I_{2 \text { (current) }}$ represent the moment of inertia with respect to the neutral axis in $x_{2}$ direction for the intact ship and damaged ship, respectively. At each load step, material points with damage coefficients $\phi \geq 0.3$ are determined and they are defined as damaged parts of the ship structure. Therefore, neutral axis locations and moment of inertia $I_{2 \text { (current) }}$ values are recalculated based on the remaining intact parts.

As shown in Fig. 22, the maximum bending moment for the intact ship captured by PD is $M_{2 \max }=9.28 \times 10^{5} \mathrm{~N} . \mathrm{m}$ which agrees very well with the experimental result of $M_{2 \max (\text { experiemnt) }}=9.2705 \times 10^{5}$ N.m conducted by Nishihara (1984). As it can also be observed from the loading history curve for case 1 , shown in blue, there is a stage that the bending moment drops suddenly from $4.65 \times 10^{5}$ N.m to $2.38 \times 10^{5}$ N.m while the moment of inertia is not much reduced. This is the stage that all the shells on ship deck which are numbered 3,11 , 12,13 , are collapsed and the crack starts propagating along two sides of the ship structure. Therefore, at this stage, the ship structure is much weakened and the required bending moment is much reduced. For case 2, shown in red, due to initial cut-out, the moment of inertia of the ship cross-section is initially reduced by $12.6 \%$ compared to the intact ship. The maximum bending moment captured by PD is $M_{2 \max }=3.85 \times 10^{5} \mathrm{~N}$.m which is equal to $41.49 \%$ of the maximum bending moment for the intact case. For case 3, due to bigger cut-out, the initial reduction of the moment of inertia is $27.4 \%$ and the maximum bending moment is $M_{2 \max }=2.47 \times 10^{5} \mathrm{~N} . \mathrm{m}$ which is equal to $26.62 \%$ of the maximum bending moment for the intact case. Moreover, the moment of inertia of the final damaged ship in both cases 2 and 3 decreases by more than 55\% as shown in Fig. 22.

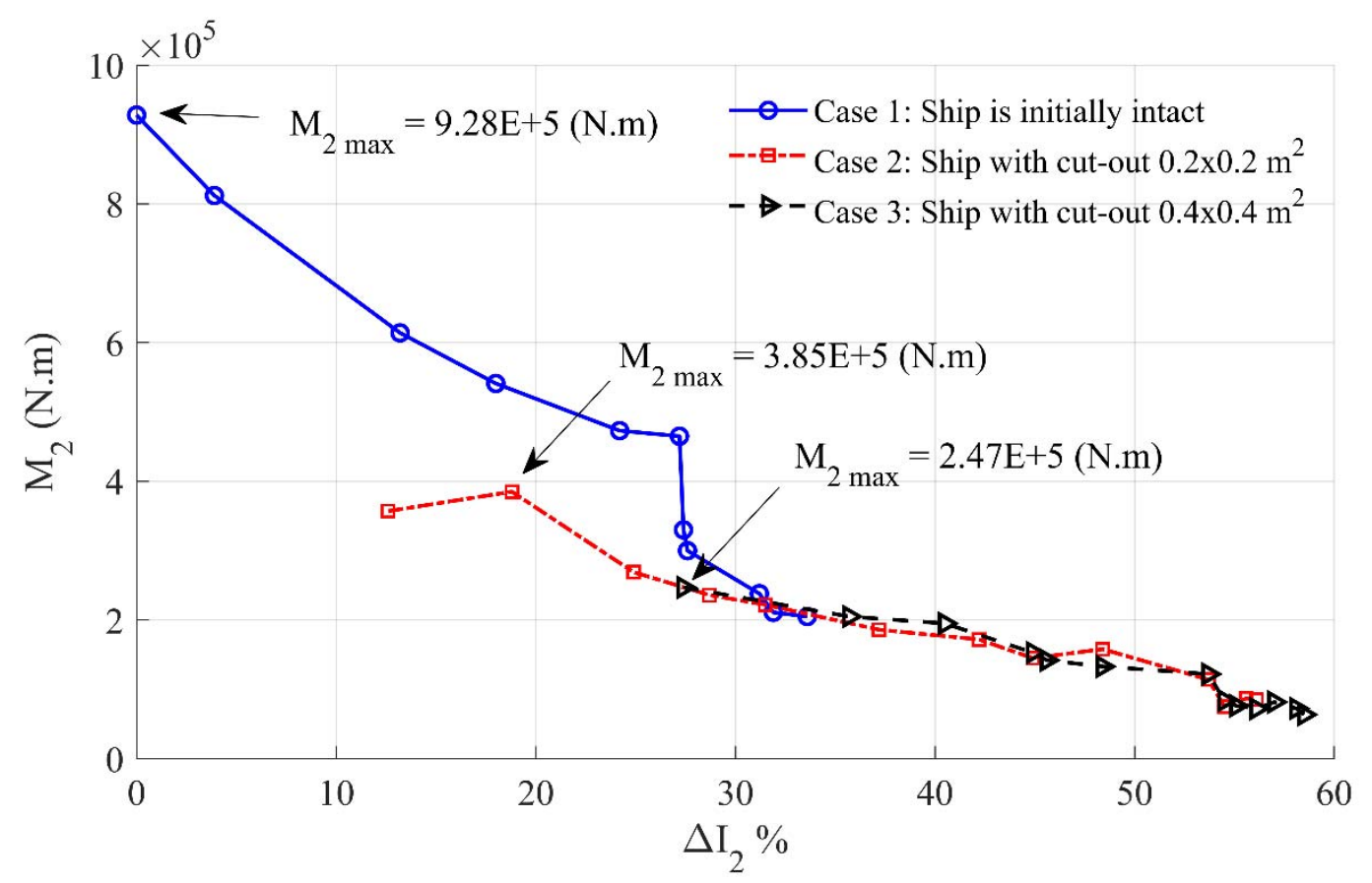

Fig. 22. Variations of the critical bending moment versus change in the moment of inertia for the ship structure 


\subsubsection{Ship structure subjected to torsional loading}

After investigating the ship model in bending condition, the ship with $0.2 \times 0.2 \mathrm{~m}^{2}$ cut-out is further investigated for the torsional loading condition as shown in Fig. 12(b). The left end of the ship is fixed and the right end of the ship is subjected to torsional loading, $M_{1}$ with respect to the neutral axis of the ship in $x_{1}$ direction. Similar to the previous example, it is assumed that the right end of the ship structure is attached to a rigid plate. The torsional moment $M_{1}$ is applied by applying nodal forces as shown in Fig. 23 as

$\mathbf{f}_{(k)}=f_{(k)} \mathbf{e}_{f(k)}$

where $f_{(k)}$ represents the magnitude and $\mathbf{e}_{f(k)}$ represents a unit vector of the nodal force $\mathbf{f}_{(k)}$. The magnitude of the nodal force is calculated as

$$
f_{(k)}=\frac{M_{1}}{N_{R}} \frac{1}{d_{(k)}}
$$

where $M_{1}$ represents the applied torque, $N_{R}$ represents the total number of material points on the rigid plate at the right end, $d_{(k)}$ represents the distance between material point $k$ and the center of the rigid plate at $\left(x_{1}=L, x_{2}=0, x_{3}=H / 2\right)$.

The unit vector, $\mathbf{e}_{f(k)}$ in Eq. (31) can be defined as

$$
\mathbf{e}_{f(k)}=\mathbf{i}_{1} \times \mathbf{i}_{(k)}
$$

with

$$
\begin{aligned}
& \mathbf{i}_{1}=\left[\begin{array}{lll}
1 & 0 & 0
\end{array}\right]^{T} \\
& \mathbf{i}_{(k)}=\frac{\mathbf{i}_{0(k)}}{\left|\mathbf{i}_{0(k)}\right|}=\frac{\mathbf{i}_{0(k)}}{d_{(k)}} \\
& \mathbf{i}_{0(k)}=\left[\begin{array}{lll}
x_{1(k)}-L & x_{2(k)}-0 & x_{3(k)}-H / 2
\end{array}\right]^{T}=\left[\begin{array}{lll}
0 & x_{2(k)} & x_{3(k)}-H / 2
\end{array}\right]^{T}
\end{aligned}
$$

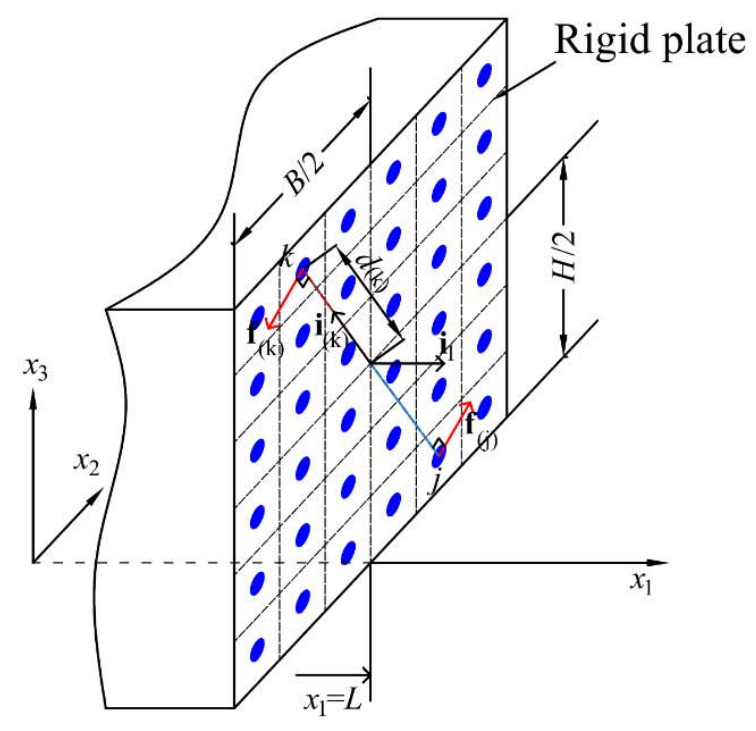

(a)

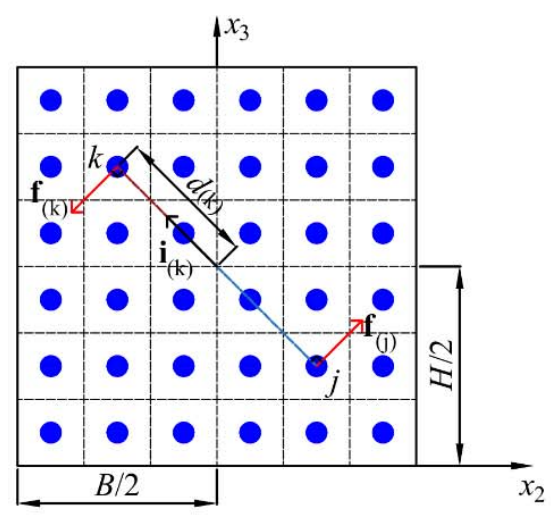

(b)

Fig. 23. Applying torque on the rigid plate by using dual forces (a): $3 \mathrm{D}$ view, (b): $2 \mathrm{D}$ view 
In the PD discretized model, the same mesh size $\Delta x=0.02 \mathrm{~m}$ is used. The critical torsional moment, $M_{1}$, at each load step is applied by the procedure presented in Appendix A. Figs. 2426 present damage evolution on the ship for torsional loading condition. As can be seen from Fig. 24, damages initiate at four corners of the cut-out when applied torque is $2.365 \times 10^{5} \mathrm{~N} . \mathrm{m}$ . At $600^{\text {th }}$ load step when applied torque is $1.699 \times 10^{5} \mathrm{~N} \cdot \mathrm{m}$, the damages propagate from four corners of the cut-out as shown in Fig. 25. At $1200^{\text {th }}$ load step when applied torque is $1.6988 \times 10^{5}$ N.m, damages propagate along with the transverse frames as shown in Fig. 26 . 


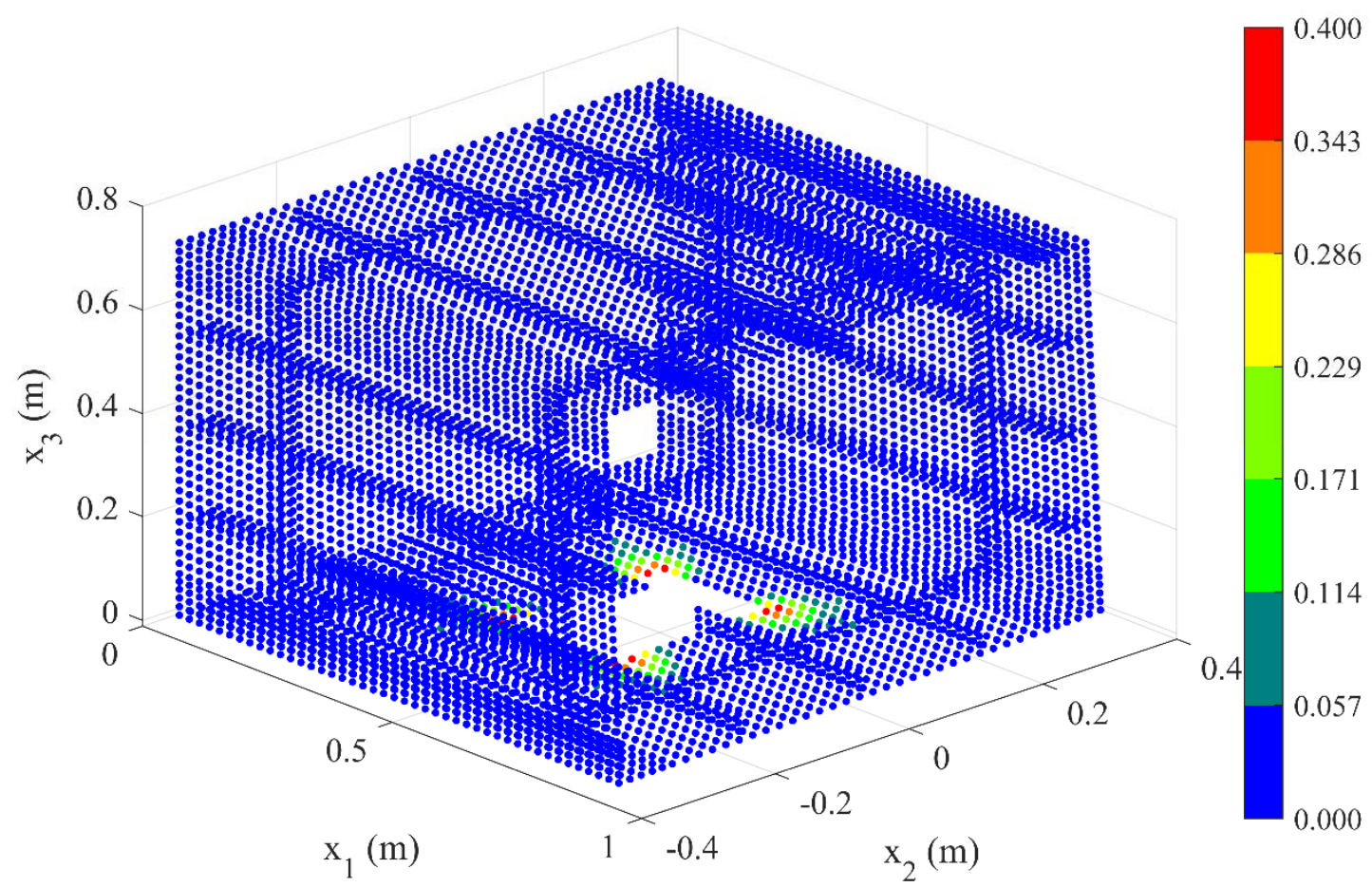

(a)

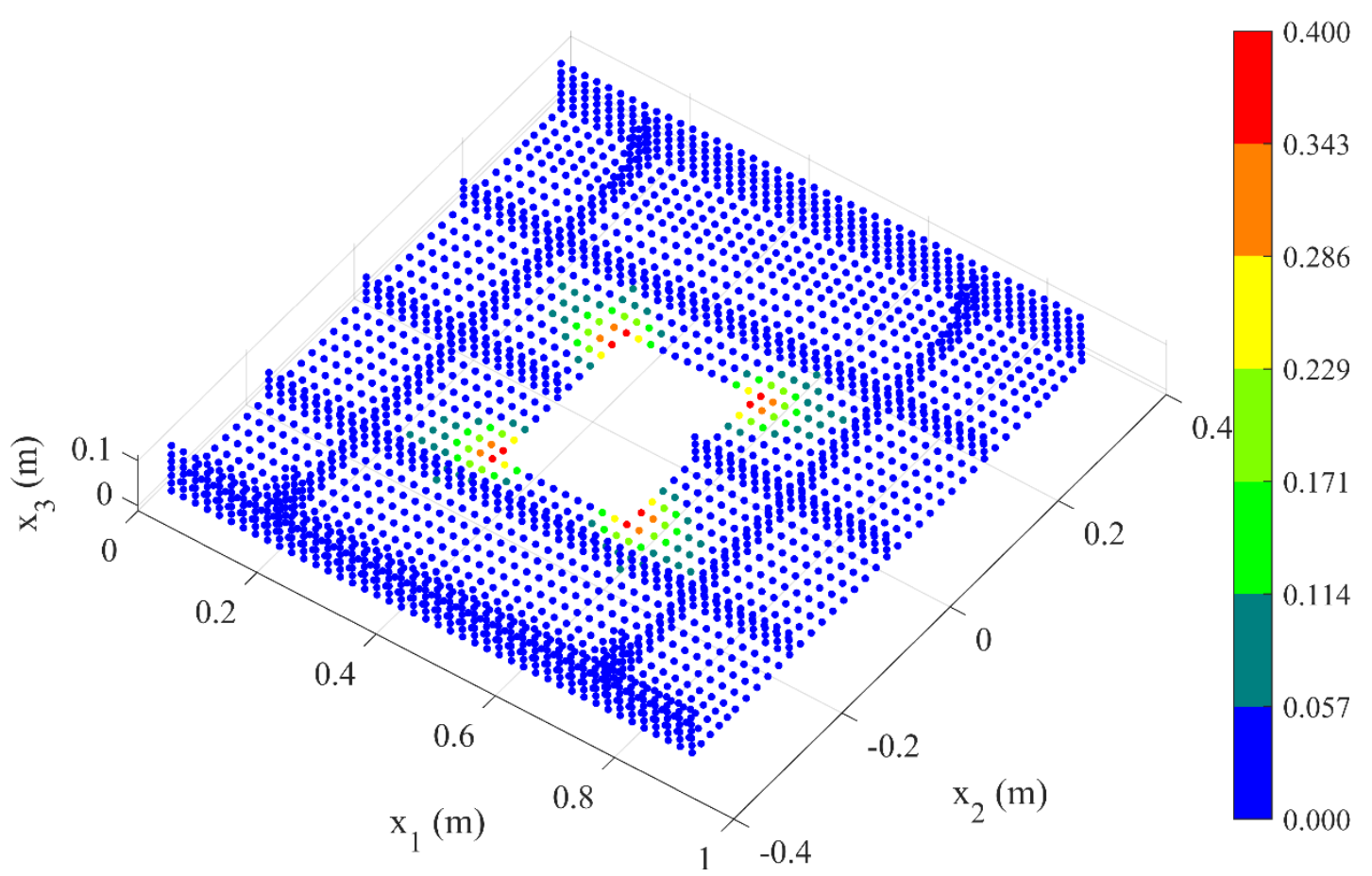

(b)

Fig. 24. Damage plot at load step $100^{\text {th }}$ when applied bending moment $M_{1}=2.365 \times 10^{5}$ N.m (a): 3D model (b): a view for material points with $x_{3} \leq 0.1 \mathrm{~m}$ (displacements are magnified 20 times for deformed configuration. Rigid plates are removed for visualization.) 


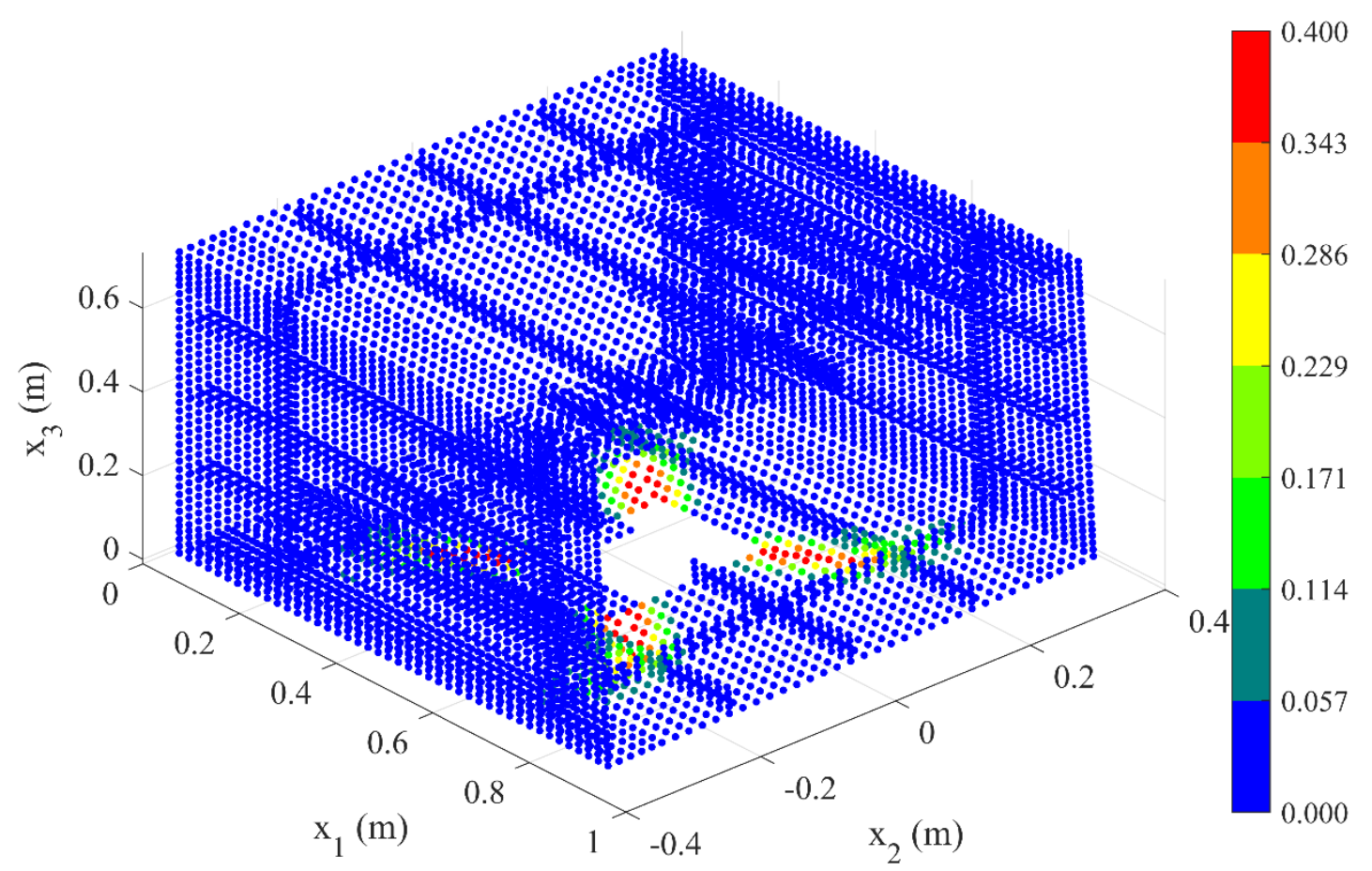

(a)

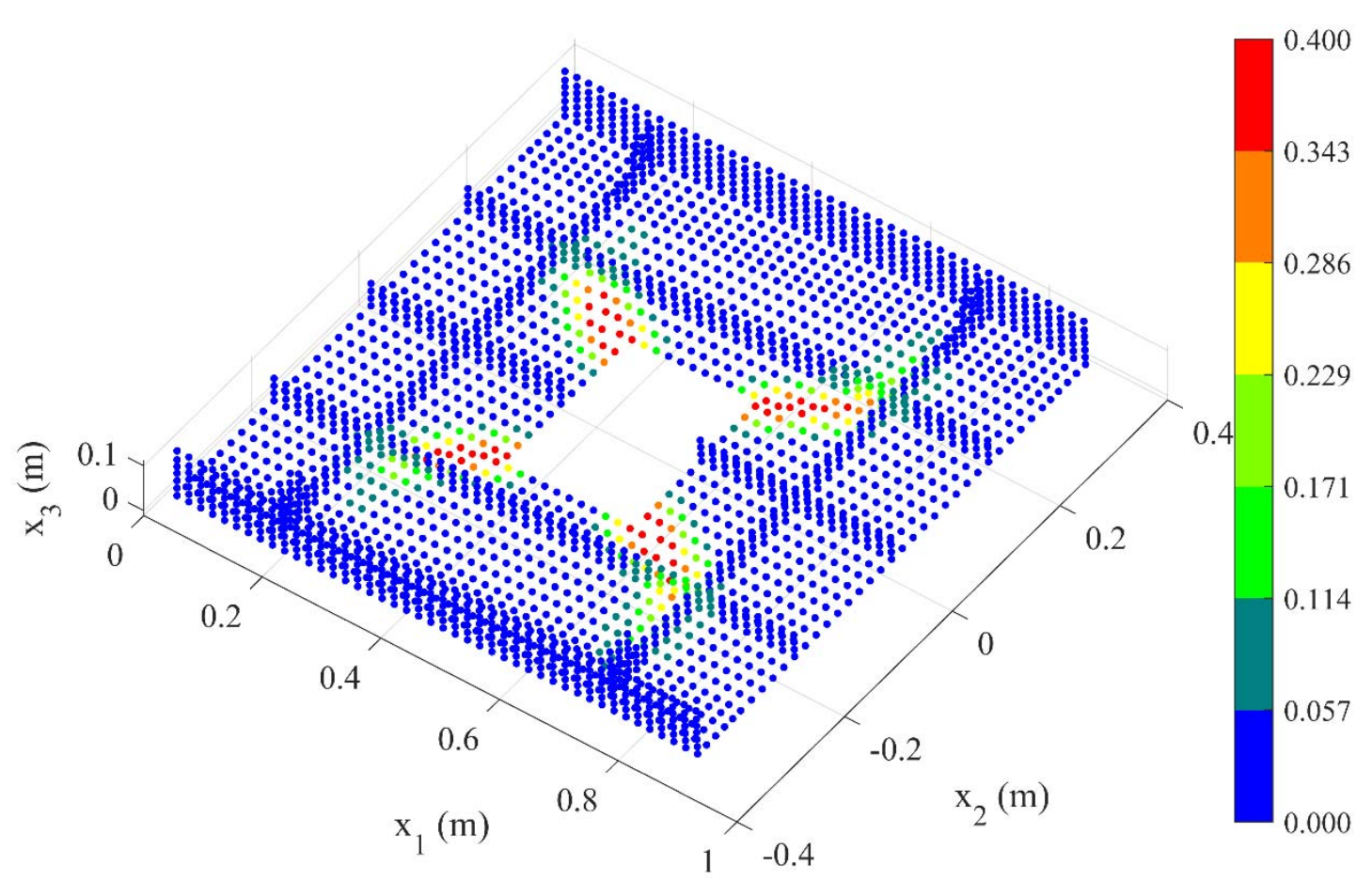

(b)

Fig. 25. Damage plot at load step $600^{\text {th }}$ when applied bending moment $M_{1}=1.699 \times 10^{5}$ N.m (a): 3D model (b): a view for material points with $x_{3} \leq 0.1 \mathrm{~m}$ (displacements are magnified 20 times for deformed configuration. Rigid plates are removed for visualization.) 


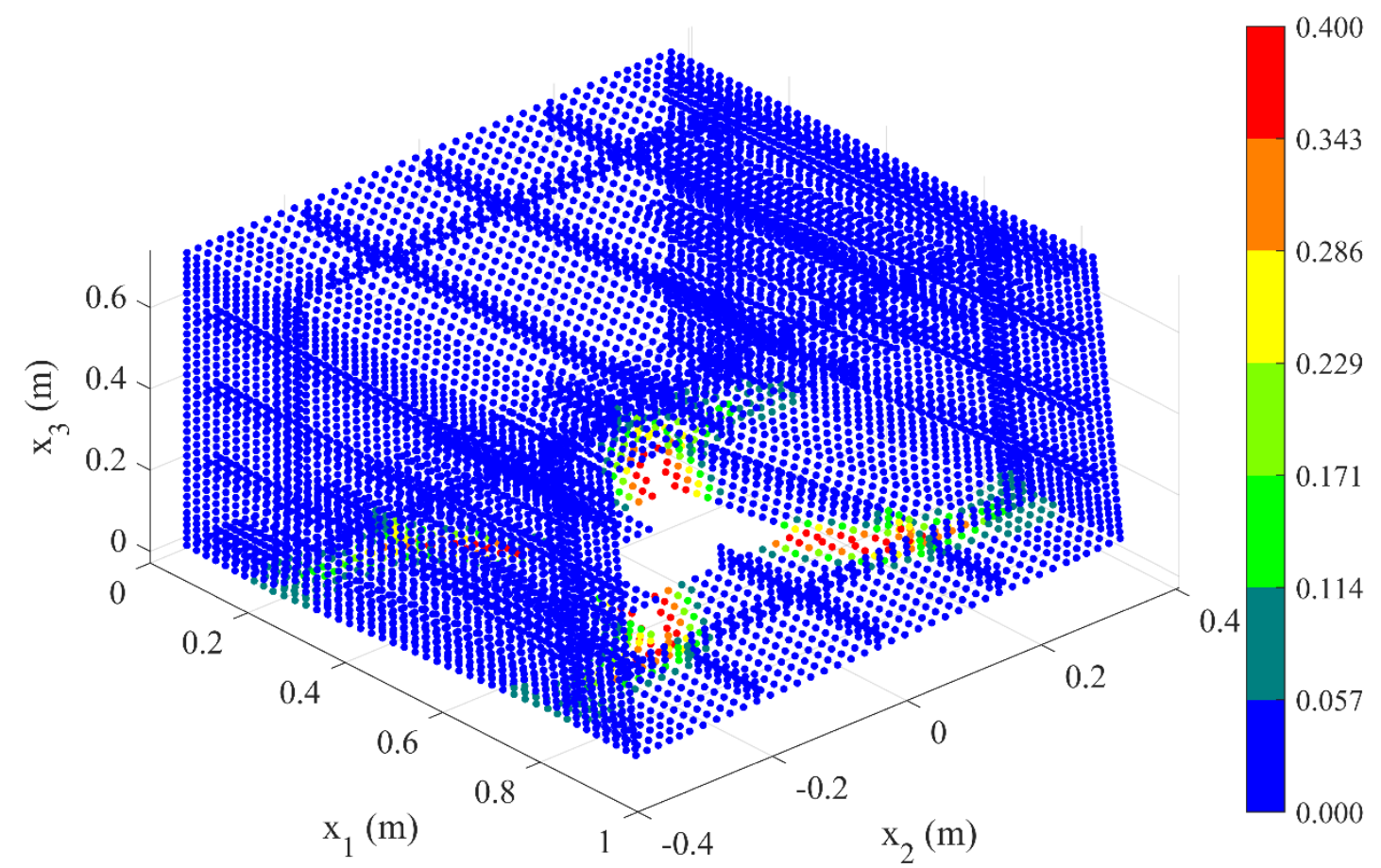

(a)

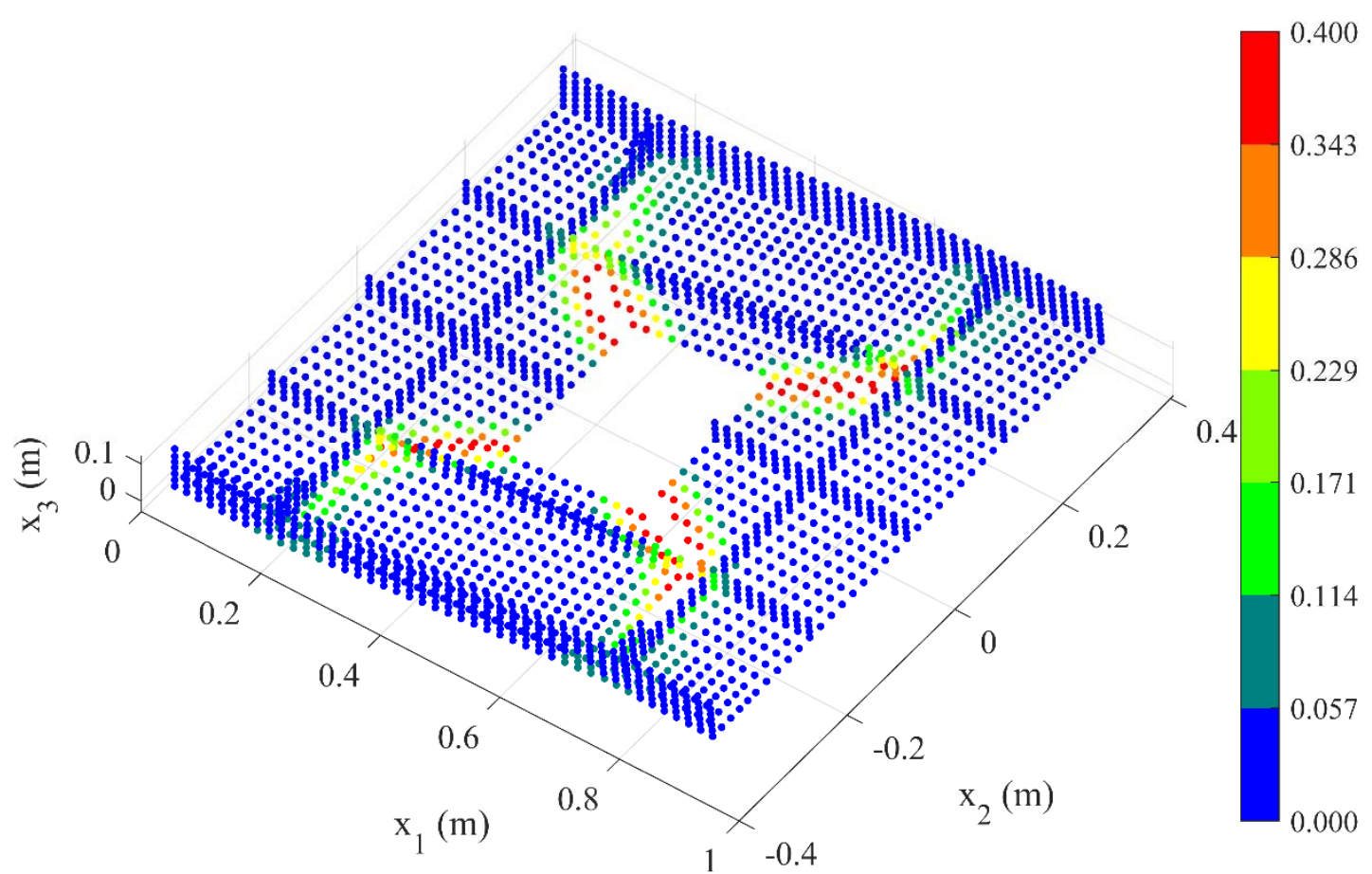

(b)

Fig. 26. Final damage plot at load step $1200^{\text {th }}$ when applied bending moment $M_{2}=1.6988 \times 10^{5}$ N.m (a): $3 \mathrm{D}$ model (b): a view for material points with $x_{3} \leq 0.1 \mathrm{~m}$ (displacements are magnified 20 times for deformed configuration. Rigid plates are removed for visualization)

Fig. 27 shows the loading history and the corresponding progressive damages on the bottom of the ship. In this figure, point (I) is associated with $600^{\text {th }}$ load step when applied torque equal 
to $1.699 \times 10^{5}$ N.m. Point (II) and (III) are associated with $800^{\text {th }}$ and $1200^{\text {th }}$ load steps when applied torques are $1.324 \times 10^{5} \mathrm{~N} . \mathrm{m}$ and $1.6988 \times 10^{5} \mathrm{~N} . \mathrm{m}$, respectively. As it can be seen from the figure, the applied torque is decreased from the initial value of $2.552 \times 10^{5} \mathrm{~N}$.m to $1.699 \times 10^{5}$ N.m (point I) after 600 load steps. At $600^{\text {th }}$ load step (point I), cracks propagate on the shell (1), from 4 corners of the cut-out to two longitudinal shells, (5) and (7) (as shown in Fig.11). Small vertical cracks also appeared on the longitudinal frames.

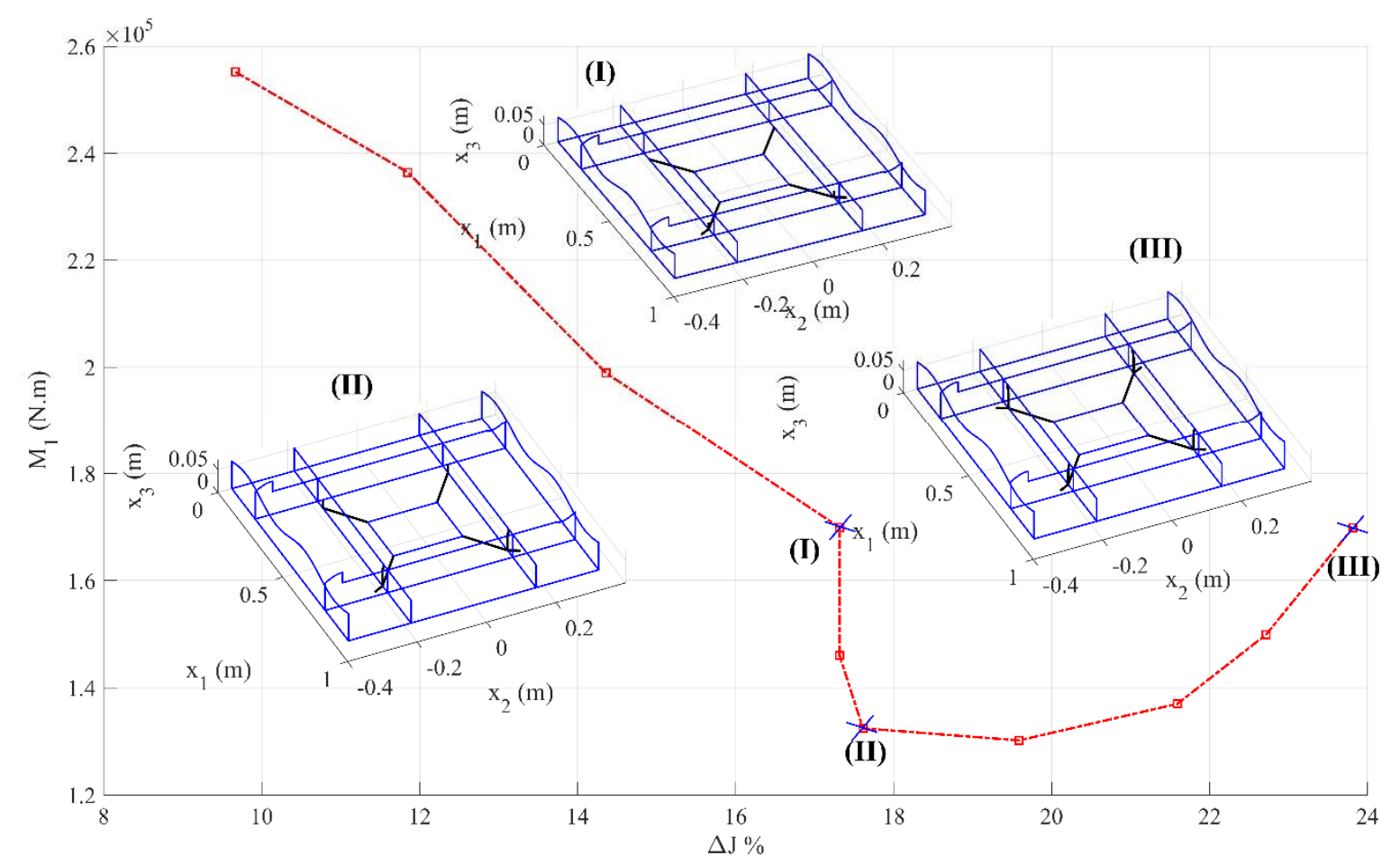

Fig. 27. Variations of the critical torsional moment, $M_{1}$ versus change in the polar moment of inertia for the ship structure with $0.2 \times 0.2 \mathrm{~m}^{2}$ cut-out

It is also observed from Fig. 27 that the applied torque is sharply reduced from $1.699 \times 10^{5}$ N.m at point (I) to $1.324 \times 10^{5} \mathrm{~N} . \mathrm{m}$ at point (II). Later, the applied torque is increased to $1.6988 \times 10^{5}$ N.m at point (III). In order to analyse these interesting phenomena, details of the crack paths up to these points are shown in Fig. 28. In this figure, the blue lines represent the edges of the cut-out. The scatter data represents the material points which have the local damage index, $\phi$ exceeding 0.2 . The black lines represent the possible crack paths on the ship structure. These crack paths are defined based on the local damage index, $\phi$ and the crack openings in the deformed configurations.

As shown in Fig. 28, the damage initiate at four corners A, B, C, D of the cut-out. By checking the damage plots every 20 load steps, it is observed that the damages start at the corners $\mathrm{C}$ and D first. Later, damages also appear at the corners A and B. As shown in Fig. 28(a), after 600 load steps, the damages propagate from two corners $\mathrm{C}$ and $\mathrm{D}$ to the locations of $\left(x_{1}=0.7 \mathrm{~m}, x_{2}=0.21 \mathrm{~m}, x_{3}=0\right)$ and $\left(x_{1}=0.7 \mathrm{~m}, x_{2}=-0.21 \mathrm{~m}, x_{3}=0\right)$, respectively. There are also two short vertical cracks on the longitudinal shells (shells 5 and 7 in Fig. 11) at the locations of $\left(x_{1}=0.68 \mathrm{~m}, x_{2}= \pm 0.18 \mathrm{~m}, 0 \leq x_{3} \leq 0.0167 \mathrm{~m}\right)$. Moreover, the damages also 
propagate from two corners A and B to the locations of ( $\left.x_{1}=0.24 \mathrm{~m}, x_{2}=0.168 \mathrm{~m}, x_{3}=0\right)$ and $\left(x_{1}=0.24 \mathrm{~m}, x_{2}=-0.168 \mathrm{~m}, x_{3}=0\right)$, respectively.

From $600^{\text {th }}$ load step (point (I) in Fig. 27) to $800^{\text {th }}$ load step (point (II) in Fig. 27), the cracks propagate on the bottom and vertical shells by very short distances (approximately equal to $\Delta x$ ) as shown in Fig. 28(b). Since the vertical cracks are already created at $600^{\text {th }}$ load step, the required torques to continue propagating small amounts of cracks are much reduced from point (I) to point (II) as shown in Fig. 27.

At $1200^{\text {th }}$ load step (point (III) in Fig. 27), there are two new vertical cracks compared to the damages at $800^{\text {th }}$ load step (point (II) in Fig. 27) as shown in Fig. 28(c). The locations of these new vertical cracks are $\left(x_{1}=0.21 \mathrm{~m}, x_{2}= \pm 0.18 \mathrm{~m}, 0 \leq x_{3} \leq 0.05 \mathrm{~m}\right)$ on the longitudinal shells (shells 5 and 7 in Fig. 11). Moreover, the vertical cracks observed from $600^{\text {th }}$ and $800^{\text {th }}$ load steps also propagate to the locations of $\left(x_{1}=0.68 \mathrm{~m}, x_{2}= \pm 0.18 \mathrm{~m}, 0 \leq x_{3} \leq 0.05 \mathrm{~m}\right)$. Note that all vertical cracks reach the height of $0.05 \mathrm{~m}$ which means the longitudinal shells (shells 5 and 7 in Fig. 11) are completely damaged at $1200^{\text {th }}$ load step.

In addition, it can be observed from Fig. 28 that the cracks initiating from corners $\mathrm{C}$ and $\mathrm{D}$ propagate toward the free end of the ship where the loading conditions are applied. On the other hand, the cracks initiating from two corners A and B propagate toward the fixed end of the ship where the boundary conditions are applied. The fixed end and the free end of the ship are shown in Fig. 12(b). Moreover, it can also be observed that the cracks initiating from corners C and D propagate quicker than the cracks initiating from corners A and B. In other words, breaking the structures near the fixed end is more difficult than breaking the structures near the free end. This observation can be the reason for the increase of the required torques from $1.324 \times 10^{5}$ N.m for the point (II) to $1.6988 \times 10^{5}$ N.m for the point (III) as shown in Fig. 27.

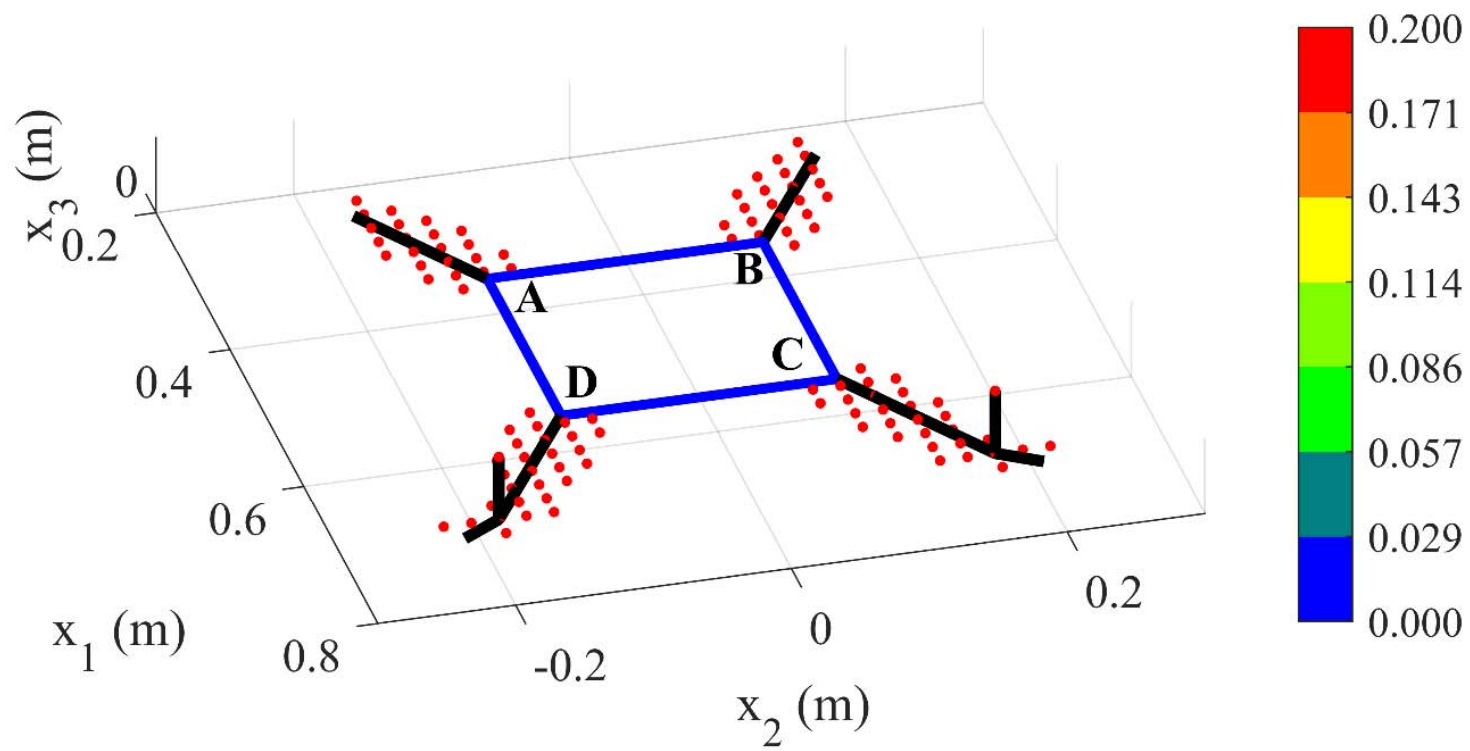

(a) 


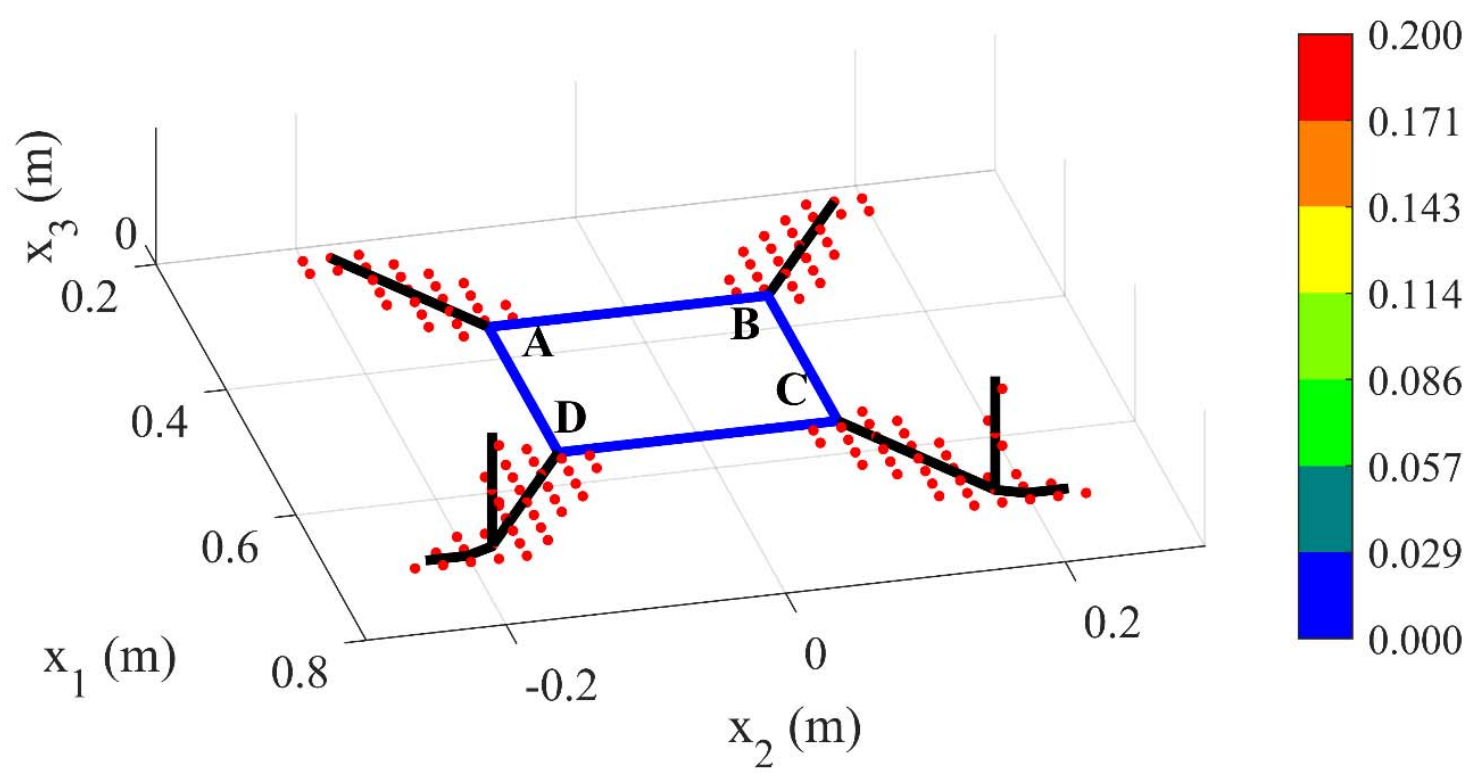

(b)

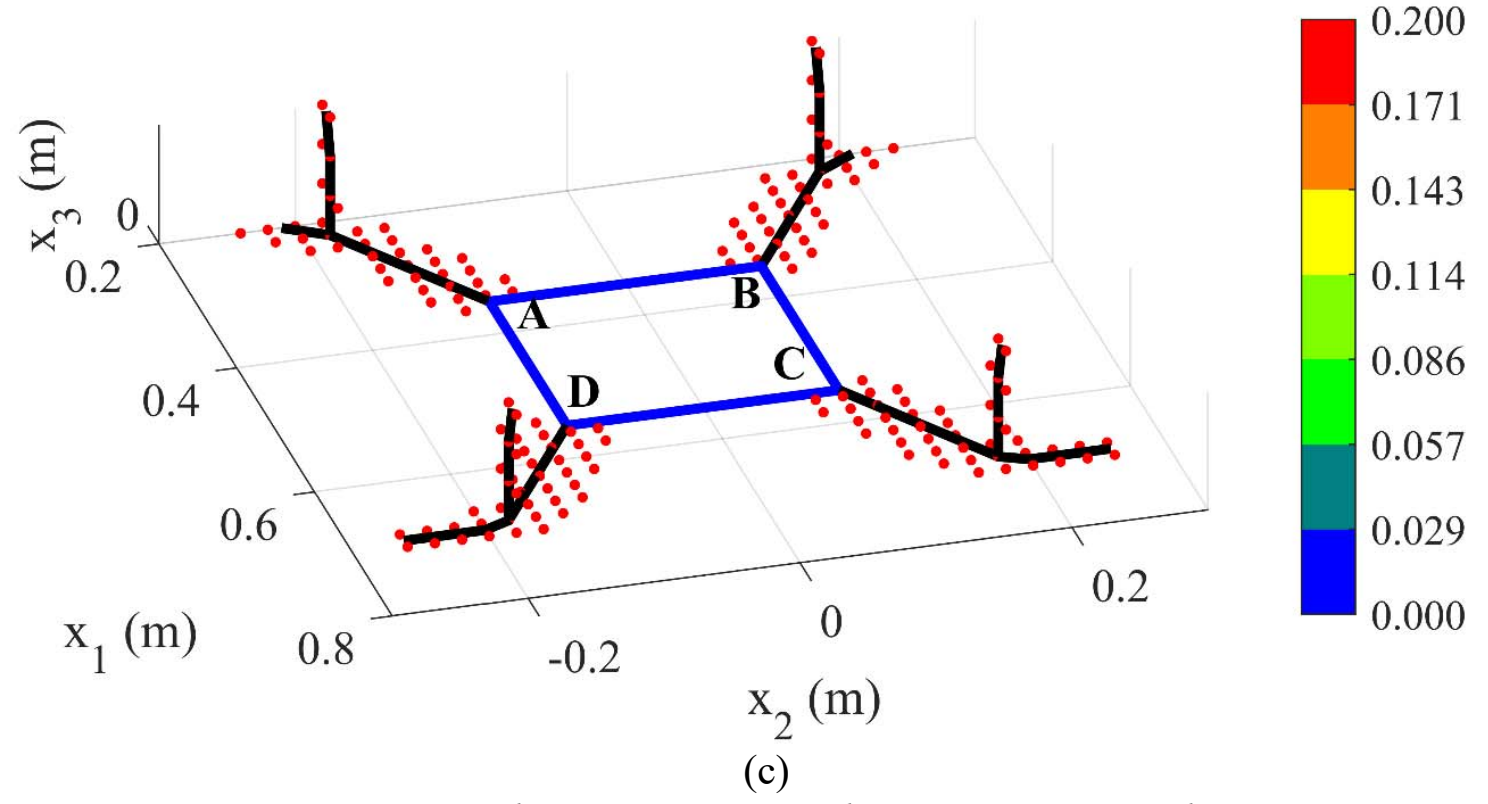

Fig. 28. Crack paths at (a) $600^{\text {th }}$ (point I), (b) $800^{\text {th }}$ (point II), (c) $1200^{\text {th }}$ (point III) load step

\section{Conclusion}

In this study, progressive damages on ship structures are predicted by using the peridynamic shell model for the first time in the literature. The numerical procedure to predict damages on stiffened structures is also provided. The progressive damages on a plate subjected to antitearing load show a good agreement with experimental results.

The progressive damages on the MST4 ship model studied by Nishihara (1984) subjected to the bending condition are predicted. It is observed that there is a good agreement between PD and experimental results in terms of the maximum value of the bending moment. The damage propagation in the ship structure is also further investigated by considering torsional loading conditions. 
It is observed that in the sagging condition with no initial damage, the top part of the ship structure is subjected to compressive load. As a result, buckling occurs and the structure is damaged in this region. For the ship structure with a rectangular cut-out on the bottom, the damage initiates on four corners of the cut-out. Then, cracks propagate beyond the longitudinal shells on the ship bottom and go towards the ship sides at the locations next to transverse shells. Finally, the cracks propagate vertically along with the transverse shells on two sides of the ship structure.

In the torsional loading condition, the damage also initiates on four corners of the cut-out. Then, the cracks propagate towards the intersections of longitudinal and transverse shells on the ship bottom before going along the transverse shells toward two sides of the ship structure. It is also found that when the cracks reach the intersections of longitudinal and transverse shells at the bottom, the critical applied torque is sharply reduced.

The variations of the structural capacity during the progressive damages are also presented as shown in Fig. 10, Fig. 22 and Fig. 27. These figures can show how much the structural strengths are reduced as damages progress. Additionally, the remaining structural strengths can also be estimated by using these figures.

For further reducing the computational cost, the PD shell model can be combined with the existing PD beam models (O'Grady and Foster (2014a), Diyaroglu et al. (2015), Diyaroglu et al. (2019), Nguyen and Oterkus (2019c)) to predict possible damages and the remaining strengths of ships and offshore structures.

\section{Acknowledgment}

The authors gratefully acknowledge the support from the Ministry of Education and Training in Vietnam and the University of Strathclyde. Results were obtained using the ARCHIE-WeSt High Performance Computer (www.archie-west.ac.uk) based at the University of Strathclyde.

\section{Appendix A. Evaluation of the critical loading}

Evaluation of critical loading for a structure is numerically calculated by considering damage propagation. The critical bending and torsional moments are evaluated by using the following procedure:

At each load step, the quasi-static loading condition is assumed for the structure. As utilised by Nguyen and Oterkus (2019b), the PD solution for shell structures subjected to quasi-static loading conditions can be obtained by using the adaptive dynamic relaxation method (Kilic and Madenci, 2010b; Underwood, 1986). Therefore, the equation of motion in Eq. (17b) can be rewritten as

$$
\overline{\mathbf{M}}_{(k)} \ddot{\mathbf{u}}_{(k)}^{G}+\mathbf{C}_{(k)} \dot{\mathbf{u}}_{(k)}^{G}=\sum_{j=1}^{N} \mu_{(k)(j)} \mathbf{F}_{(k)(j)}^{G} V_{(j)}+\mathbf{B}_{(k)}^{G}
$$

where $\mathbf{C}_{(k)}$ and $\overline{\mathbf{M}}_{(k)}$ represent the damping matrix and the mass stable vector, respectively (Nguyen and Oterkus, 2019b). The term $\dot{\mathbf{u}}_{(k)}^{G}$ represents the velocity vector

In order to determine the critical loading for each load step, a trial loading, $\mathbf{B}_{0}^{G}$ (unit forces or unit bending moments) is applied to the structure as 


$$
\mathbf{B}_{0}^{G}=\left[\begin{array}{llllll}
F_{1} & F_{2} & F_{3} & M_{1} & M_{2} & M_{3}
\end{array}\right]^{T}
$$

The corresponding displacement field, $\mathbf{U}_{0}^{G}$, for the trial loading is obtained by solving Eq. (A1), and the energy release rate for each interaction, $\bar{g}_{(k)(j)}$, is calculated by using Eq. (21). The maximum value of the energy release rate of the model, $\bar{g}_{\max }$, is determined as $\bar{g}_{\max }=\max \left(\bar{g}_{(k)(j)}\right): k, j=1, \ldots, N_{p}$

The ratio between the critical energy release rate and $\bar{g}_{\max }$ is calculated as $c_{0}=\sqrt{\frac{g_{c}}{\bar{g}_{\max }}}$

Next, the critical loading, $\mathbf{B}^{G}$, can be defined by scaling the trial loading by $c_{0}$ as $\mathbf{B}^{G}=c_{0} \mathbf{B}_{0}^{G}$

and the corresponding displacement field, $\mathbf{U}^{G}$, is obtained by solving Eq. (17b) and the energy release rate for each interaction, $\bar{g}_{(k)(j)}$ is re-calculated by using Eq. (21). The state of interaction is also updated by using Eq. (18).

Fig. 28 summarizes the procedure for finding the critical loads. By using this procedure, the critical values of loads are determined for each load step. 


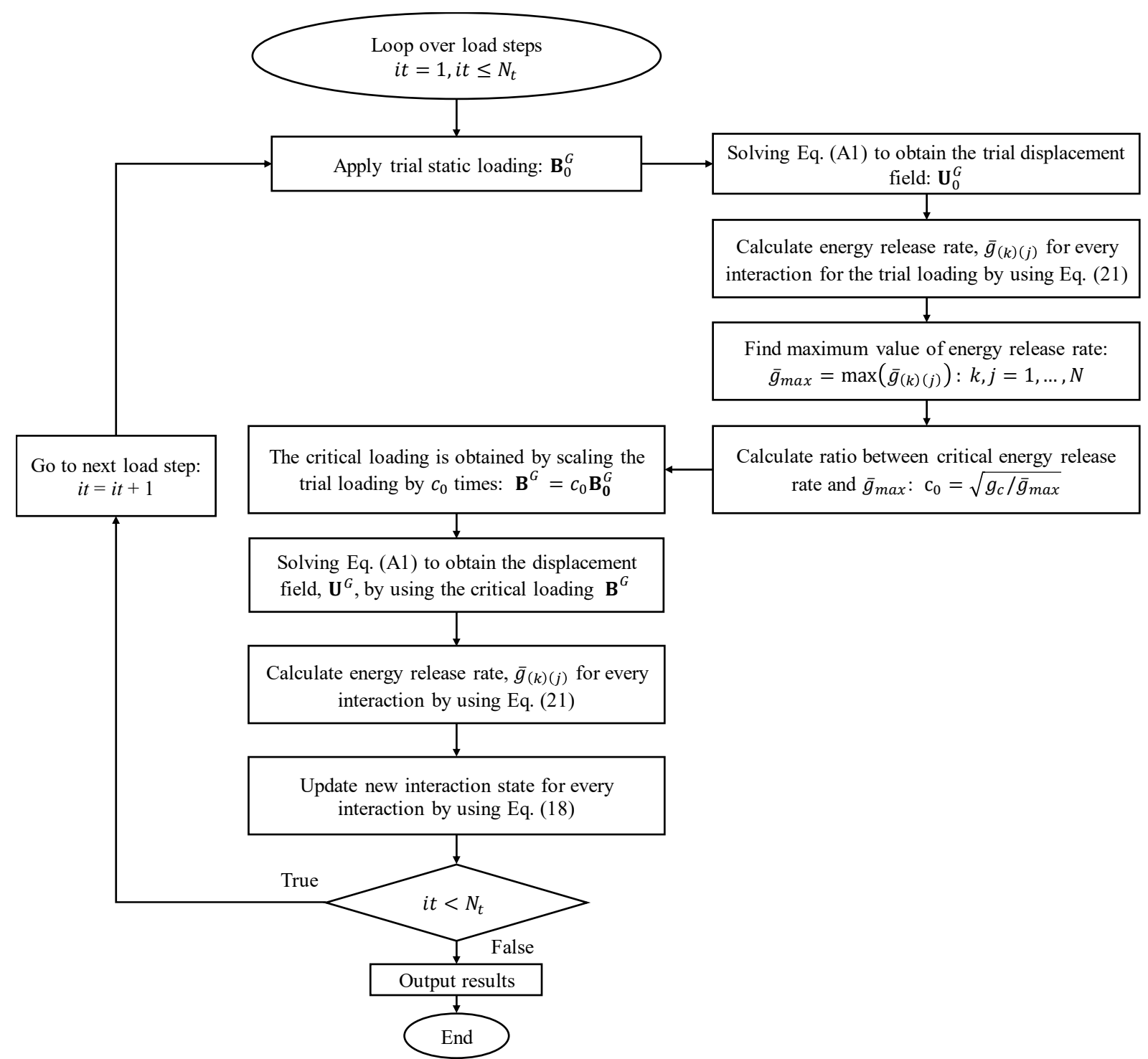

Fig. 29. Procedure for finding critical loads

\section{Appendix B. Calculation of von Mises stresses}

In this section, the calculation of von Mises stresses in PD is presented. The von Mises stresses in PD can be calculated by using the same formulations used in classical continuum mechanics(CCM). In CCM, the von Mises stresses can be calculated as (Boresi et al., 1985)

$$
\sigma_{V M}=\sqrt{\frac{1}{2}\left[\left(\sigma_{x x}-\sigma_{y y}\right)^{2}+\left(\sigma_{y y}-\sigma_{z z}\right)^{2}+\left(\sigma_{z z}-\sigma_{x x}\right)^{2}\right]+3\left(\tau_{x y}^{2}+\tau_{y z}^{2}+\tau_{z x}^{2}\right)}
$$

For a shell structure, by assuming the plane stress condition and by considering the stresses on the shell surface $(z=h / 2)$, the followings stress components vanish

$$
\begin{aligned}
& \sigma_{z z} \approx 0 \\
& \tau_{y z}=0 ; \tau_{z x}=0 \text { at } z=h / 2
\end{aligned}
$$

Therefore, by substituting Eq. (B2) into Eq. (B1), the von Mises stresses for a shell structure at the shell surface $(z=h / 2)$ can be calculated as 
$\sigma_{V M}=\sqrt{\frac{1}{2}\left[\left(\sigma_{x x}-\sigma_{y y}\right)^{2}+\sigma_{y y}^{2}+\sigma_{x x}^{2}\right]+3 \tau_{x y}^{2}}$

where, the stress components $\sigma_{x x}, \sigma_{y y}, \sigma_{x y}$ on the shell surface, $z=h / 2$ can be calculated as (Nguyen and Oterkus, 2019b)

$$
\begin{aligned}
& \sigma_{x x}=\frac{E}{1-v^{2}}\left[u_{, x}+\frac{h}{2} \theta_{y, x}+v\left(v_{y y}-\frac{h}{2} \theta_{x, y}\right)\right] \\
& \sigma_{y y}=\frac{E}{1-v^{2}}\left[v_{y y}-\frac{h}{2} \theta_{x, y}+v\left(u_{, x}+\frac{h}{2} \theta_{y, x}\right)\right] \\
& \sigma_{x y}=\frac{E}{2(1+v)}\left[u_{, y}+v_{, x}+\frac{h}{2}\left(\theta_{y, y}-\theta_{x, x}\right)\right]
\end{aligned}
$$

In $\mathrm{PD}$, the stress components and the von Mises stresses for each material point can also be calculated by using the formulation given in Eq. (B3) and (B4) as

$$
\sigma_{V M(k)}=\sqrt{\frac{1}{2}\left[\left(\sigma_{x x(k)}-\sigma_{y y(k)}\right)^{2}+\sigma_{y y(k)}^{2}+\sigma_{x x(k)}^{2}\right]+3 \tau_{x y(k)}^{2}}
$$

with

$$
\begin{aligned}
& \sigma_{x x(k)}=\frac{E}{1-v^{2}}\left[u_{, x(k)}+\frac{h}{2} \theta_{y, x(k)}+v\left(v_{, y(k)}-\frac{h}{2} \theta_{x, y(k)}\right)\right] \\
& \sigma_{y y(k)}=\frac{E}{1-v^{2}}\left[v_{, y(k)}-\frac{h}{2} \theta_{x, y(k)}+v\left(u_{, x(k)}+\frac{h}{2} \theta_{y, x(k)}\right)\right] \\
& \sigma_{x y(k)}=\frac{E}{2(1+v)}\left[u_{, y(k)}+v_{, x(k)}+\frac{h}{2}\left(\theta_{y, y(k)}-\theta_{x, x(k)}\right)\right]
\end{aligned}
$$

In Eq. (B5), the derivatives, $u_{, x(k)}, u_{, y}, v_{, x(k)}, v_{y, y(k)}, \theta_{x, x(k)}, \theta_{x, y(k)}, \theta_{y, x(k)}, \theta_{y, y(k)}$ is obtained by using the displacement gradient, $\nabla \mathbf{u}_{(k)}^{L}$ described by Breitenfeld et al., (2014).

In this study, the damage parameter, $\mu$ is also included in the displacement gradient proposed by Breitenfeld et al. (2014) as

$\nabla \mathbf{u}_{(k)}^{L}=\left[\sum_{j=1}^{N} \mu_{(k)(j)}\left(\frac{\delta}{\left|\mathbf{x}_{(j)}-\mathbf{x}_{(k)}\right|}\right)^{2}\left(\mathbf{u}_{(j)}^{L}-\mathbf{u}_{(k)}^{L}\right) \otimes\left(\mathbf{x}_{(j)}-\mathbf{x}_{(k)}\right) V_{(j)}\right] \mathbf{K}_{(k)}$

where $\mathbf{x}_{(k)}=\left[\begin{array}{lll}x_{(k)} & y_{(k)} & z_{(k)}\end{array}\right]^{T}$ represents the vector of the local coordinates of material point $k$. The term $\mathbf{K}_{(k)}$ represents the shape function at material point $k$ and it can be defined as $\mathbf{K}_{(k)}=\left[\sum_{j=1}^{N} \mu_{(k)(j)}\left(\frac{\delta}{\left|\mathbf{x}_{(j)}-\mathbf{x}_{(k)}\right|}\right)^{2}\left(\mathbf{x}_{(j)}-\mathbf{x}_{(k)}\right) \otimes\left(\mathbf{x}_{(j)}-\mathbf{x}_{(k)}\right) V_{(j)}\right]^{-1}$ 


\section{References}

AbuBakar, A., Dow, R., 2013. Simulation of ship grounding damage using the finite element method. International Journal of Solids and Structures 50 (5), 623-636.

Allianz Global Corporate \& Specialty SE. 2019. Safety and Shipping Review 2019. An annual review of trends and developments in shipping losses and safety. Munich, Germany: Allianz Global Corporate \& Specialty SE.

Alpay, S., Madenci, E., 2013. Crack growth prediction in fully-coupled thermal and deformation fields using peridynamic theory, 54th AIAA/ASME/ASCE/AHS/ASC structures, structural dynamics, and materials conference, p. 1477.

Askari, E., Bobaru, F., Lehoucq, R., Parks, M., Silling, S., Weckner, O., 2008. Peridynamics for multiscale materials modeling, Journal of Physics: Conference Series. IOP Publishing, p. 012078.

Benac, D.J., Shaffer, D. and Wood, D., 2015. Managing Cold Temperature and Brittle Fracture Hazards in the Ammonia-Related Industries. In AlChE 60th Annual Safety in the Ammonia Plants \& Related Facilities Symposium (August 30-September 3, 2015).

Benac, D. J., Cherolis, N. \& Wood, D. 2016. Managing cold temperature and brittle fracture hazards in pressure vessels. Journal of Failure Analysis and Prevention, 16, 55-66.

Benson, S., Downes, J., Dow, R.S., 2013. Compartment level progressive collapse analysis of lightweight ship structures. Marine Structures 31, 44-62.

Bie, Y., Cui, X., Li, Z., 2018. A coupling approach of state-based peridynamics with nodebased smoothed finite element method. Computer Methods in Applied Mechanics Engineering 331, 675-700.

Bobaru, F., Ha, Y.D., 2011. Adaptive refinement and multiscale modeling in 2D peridynamics. Journal for Multiscale Computational Engineering, 635-659.

Boresi, A.P., Schmidt, R.J. and Sidebottom, O.M., 1985. Advanced mechanics of materials (Vol. 6). New York et al.: Wiley.

Breitenfeld, M., Geubelle, P.H., Weckner, O., Silling, S., 2014. Non-ordinary state-based peridynamic analysis of stationary crack problems. Computer Methods in Applied Mechanics Engineering 272, 233-250.

De Meo, D., Zhu, N., Oterkus, E., 2016. Peridynamic modeling of granular fracture in polycrystalline materials. Journal of Engineering Materials Technology 138 (4), 041008.

Diyaroglu, C., 2016. Peridynamics and its applications in marine structures. University of Strathclyde.

Diyaroglu, C., Oterkus, E., Oterkus, S., 2019. An Euler-Bernoulli beam formulation in an ordinary state-based peridynamic framework. Mathematics Mechanics of Solids 24 (2), 361-376.

Diyaroglu, C., Oterkus, E., Oterkus, S., Madenci, E., 2015. Peridynamics for bending of beams and plates with transverse shear deformation. International Journal of Solids Structures 69, 152-168. 
Diyaroglu, C., Oterkus, S., Oterkus, E., Madenci, E., 2017. Peridynamic modeling of diffusion by using finite-element analysis. IEEE Transactions on Components, Packaging Manufacturing Technology 7 (11), 1823-1831.

Drouin, P., 2006. Brittle fracture in ships-a lingering problem. Ships and Offshore Structures, 1(3), pp.229-233.

Foster, J.T., Silling, S.A., Chen, W.W., 2010. Viscoplasticity using peridynamics. International Journal for Numerical Methods in Engineering 81 (10), 1242-1258.

Gao, Y., Oterkus, S., 2019a. Fully coupled thermomechanical analysis of laminated composites by using ordinary state based peridynamic theory. Composite Structures 207, 397-424.

Gao, Y., Oterkus, S., 2019b. Non-local modeling for fluid flow coupled with heat transfer by using peridynamic differential operator. Engineering Analysis with Boundary Elements $105,104-121$.

Gao, Y., Oterkus, S., 2019c. Ordinary state-based peridynamic modelling for fully coupled thermoelastic problems. Continuum Mechanics Thermodynamics 31 (4), 907-937.

Han, S., Diyaroglu, C., Oterkus, S., Madenci, E., Oterkus, E., Hwang, Y., Seol, H., 2016. Peridynamic direct concentration approach by using ANSYS, 2016 IEEE 66th Electronic Components and Technology Conference (ECTC). IEEE, pp. 544-549.

Hu, W., Ha, Y.D., Bobaru, F., 2012. Peridynamic model for dynamic fracture in unidirectional fiber-reinforced composites. Computer Methods in Applied Mechanics Engineering 217, 247-261.

Huang, Y., Oterkus, S., Hou, H., Oterkus, E., Wei, Z., Zhang, S., 2019. Peridynamic model for visco-hyperelastic material deformation in different strain rates. Continuum Mechanics Thermodynamics 1-35.

Javili, A., Morasata, R., Oterkus, E., Oterkus, S., 2018. Peridynamics review. Mathematics Mechanics of Solids, 1081286518803411.

Kalthoff, J.F., 1988. Shadow optical analysis of dynamic shear fracture. Optical Engineering 27 (10), 271035.

Kanok-nukulchai, W., 1979. A simple and efficient finite element for general shell analysis. International Journal for Numerical Methods in Engineering 14 (2), 179-200.

Kilic, B., Madenci, E., 2010a. Coupling of peridynamic theory and the finite element method. Journal of mechanics of materials structures 5 (5), 707-733.

Kilic, B., Madenci, E., 2010b. An adaptive dynamic relaxation method for quasi-static simulations using the peridynamic theory. Theoretical Applied Fracture Mechanics 53 (3), 194-204.

Kim, K. J., Lee, J. H., Park, D. K., Jung, B. G., Han, X. \& Paik, J. K. 2016. An experimental and numerical study on nonlinear impact responses of steel-plated structures in an Arctic environment. International Journal of Impact Engineering, 93, 99-115. 
Kobayashi, H. \& Onoue, H. 1943. Brittle fracture of liberty ships. Failure Knowledge Database, 100, 67.

Kyokai, N.K.J.J.N., 2014. Investigation Report on Structural Safety of Large Container Ship.

Le, Q., Bobaru, F., 2018. Surface corrections for peridynamic models in elasticity and fracture. Computational Mechanics 61 (4), 499-518.

Li, Y., Wierzbicki, T., Sutton, M.A., Yan, J., Deng, X., 2011. Mixed mode stable tearing of thin sheet AI 6061-T6 specimens: experimental measurements and finite element simulations using a modified Mohr-Coulomb fracture criterion. International Journal of Fracture 168 (1), 53-71.

Liu, W., Hong, J.-W., 2012. A coupling approach of discretized peridynamics with finite element method. Computer Methods in Applied Mechanics Engineering 245, 163-175.

Macek, R.W., Silling, S.A., 2007. Peridynamics via finite element analysis. Finite Elements in Analysis Design 43 (15), 1169-1178.

MacMaster, F., Chan, K., Bergsma, S., Kassner, M., 2000. Aluminum alloy 6069 part II: fracture toughness of 6061-T6 and 6069-T6. Materials Science Engineering: A 289 (1-2), 54-59.

Madenci, E., Oterkus, E., 2014. Peridynamic Theory and Its Applications. Springer.

Madenci, E., Oterkus, S., 2016a. Ordinary state-based peridynamics for plastic deformation according to von Mises yield criteria with isotropic hardening. Journal of the Mechanics Physics of Solids 86, 192-219.

Madenci, E., Oterkus, S., 2016b. Peridynamics for coupled field equations. Handbook of Peridynamic Modeling, 489-531.

Madenci, E., Oterkus, S., 2017. Ordinary state-based peridynamics for thermoviscoelastic deformation. Engineering Fracture Mechanics 175, 31-45.

Matthews, W.T., 1973. Plane strain fracture toughness (KIC) data handbook for metals. ARMY MATERIALS AND MECHANICS RESEARCH CENTER WATERTOWN MA.

Mitchell, J.A., 2011. A non-local, ordinary-state-based viscoelasticity model for peridynamics. Sandia National Lab Report 8064, 1-28.

Nguyen, C.T., Oterkus, S., 2019a. Ordinary state-based peridynamic model for geometrically nonlinear analysis. Engineering Fracture Mechanics.

Nguyen, C.T., Oterkus, S., 2019b. Peridynamics for the thermomechanical behavior of shell structures. Engineering Fracture Mechanics, 106623.

Nguyen, C.T., Oterkus, S., 2019c. Peridynamics formulation for beam structures to predict damage in offshore structures. Ocean Engineering 173, 244-267.

Nishihara, S., 1984. Ultimate longitudinal strength of midship cross-section. Naval architecture and ocean engineering 22, 200-214. 
O'Grady, J., Foster, J., 2014a. Peridynamic beams: a non-ordinary, state-based model. International Journal of Solids Structures 51 (18), 3177-3183.

O’Grady, J., Foster, J., 2014b. Peridynamic plates and flat shells: A non-ordinary, state-based model. International Journal of Solids Structures 51 (25-26), 4572-4579.

Oterkus, E., 2010. Peridynamic theory for modeling three-dimensional damage growth in metallic and composite structures. The University of Arizona, The University of Arizona.

Oterkus, E., Madenci, E., Weckner, O., Silling, S., Bogert, P., Tessler, A., 2012. Combined finite element and peridynamic analyses for predicting failure in a stiffened composite curved panel with a central slot. Composite Structures 94 (3), 839-850.

Oterkus, S., 2015. Peridynamics for the solution of multiphysics problems. The University of Arizona, The University of Arizona.

Oterkus, S., Fox, J., Madenci, E., 2013. Simulation of electro-migration through peridynamics, 2013 IEEE 63rd Electronic Components and Technology Conference. IEEE, pp. 14881493.

Paik, J.K., Amlashi, H., Boon, B., Branner, K., Caridis, P., Das, P., Fujikubo, M., Huang, C.H., Josefson, L., Kaeding, P., 2012. Committee III. 1 ultimate strength, 18th International ship and offshore structures congress. Schiffbautechnische Gesellschaft eV, pp. 285-363.

Sarego, G., Le, Q.V., Bobaru, F., Zaccariotto, M., Galvanetto, U., 2016. Linearized state-based peridynamics for 2-D problems. International Journal for Numerical Methods in Engineering 108 (10), 1174-1197.

Silling, S.A., 2000. Reformulation of elasticity theory for discontinuities and long-range forces. Journal of the Mechanics and Physics of Solids 48 (1), 175-209.

Silling, S.A., Askari, E., 2005. A meshfree method based on the peridynamic model of solid mechanics. Computers \& structures 83 (17-18), 1526-1535.

Silling, S.A., Bobaru, F., 2005. Peridynamic modeling of membranes and fibers. International Journal of Non-Linear Mechanics 40 (2-3), 395-409.

Silling, S.A., Lehoucq, R., 2010. Peridynamic theory of solid mechanics, Advances in applied mechanics. Elsevier, pp. 73-168.

Silling, S.A., Zimmermann, M., Abeyaratne, R., 2003. Deformation of a peridynamic bar. Journal of Elasticity 73 (1-3), 173-190.

Simonsen, B.C., Törnqvist, R., 2004. Experimental and numerical modelling of ductile crack propagation in large-scale shell structures. Marine Structures 17 (1), 1-27.

Sutar, S. S., Kale, G. S. \& Merad, S. H. 2014. Analysis of ductile-to-brittle transition temperature of mild steel. Int. J. Innov. Eng. Res. Technol., 1, 1-10.

Underwood, P., 1986. Dynamic relaxation. Computational method for transient analysis 1, 245-263. 
Yang, Z., Oterkus, E., Nguyen, C.T., Oterkus, S., 2019. Implementation of peridynamic beam and plate formulations in finite element framework. Continuum Mechanics Thermodynamics 31 (1), 301-315. 